\title{
Deloitte.
}

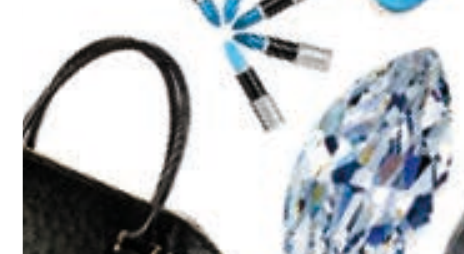

\section{Global Powers}

of Retailing 2015

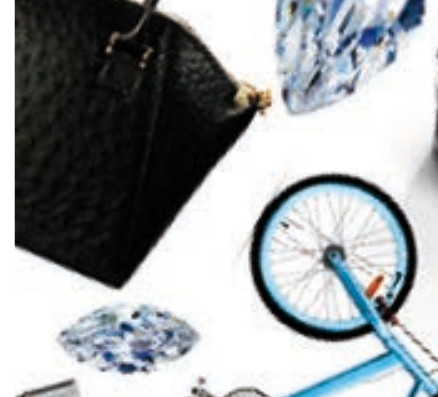

8
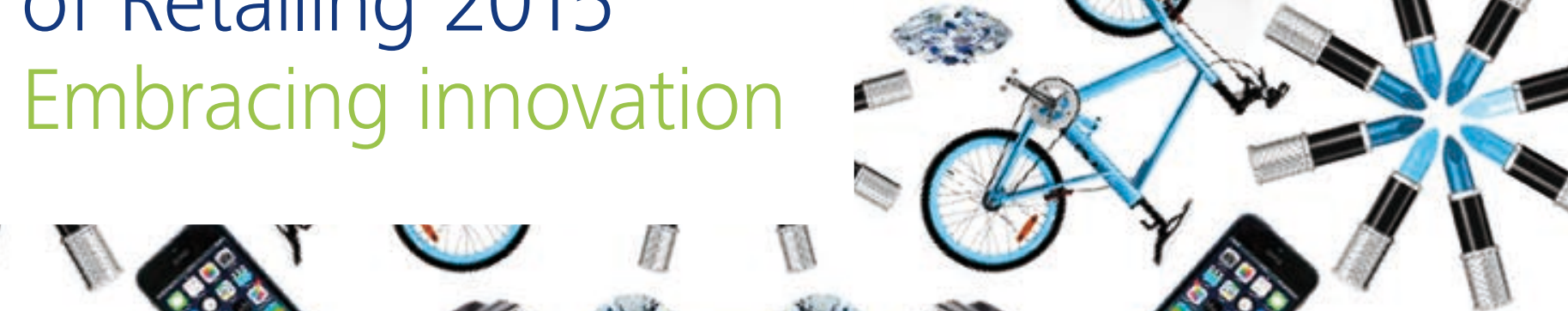

II ${ }^{\top}$. I I
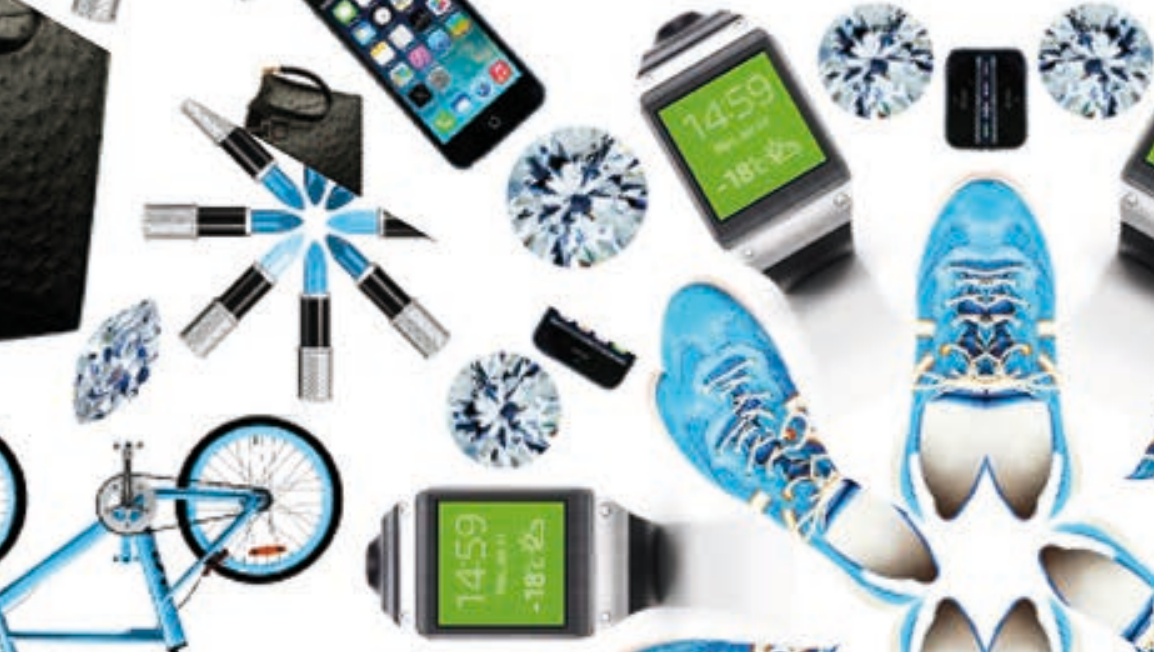

in in

L

$x$ ding

盘里

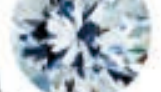

o 15

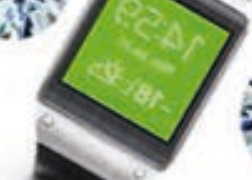

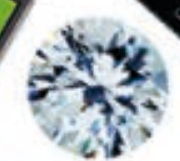

8

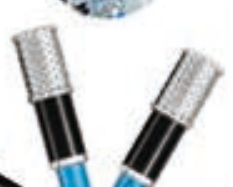




\section{Contents}

Retail trends 2015: Embracing innovation

G3

Global economic outlook

G7

Top 250 global retailers 2013

Global Powers of Retailing Top 250 highlights

Global Powers of Retailing geographical analysis

Global Powers of Retailing product sector analysis

Top 250 newcomers

Global Powers of Retailing Fastest 50

Top 50 e-retailers

Q ratio analysis for Global Powers

G37

Study methodology and data sources

G40

Contacts

G41 


\title{
Retail trends 2015: Embracing innovation
}

\author{
Welcome to Deloitte's 18th annual Global Powers of Retailing report. This report identifies \\ the 250 largest retailers around the world based on publicly available data for fiscal 2013 \\ (encompassing companies' fiscal years ended through June 2014) and analyzes their \\ performance based on geographic region, product sector, e-commerce activity, and \\ other factors.
}

\section{This year's report focuses on the theme of "embracing innovation," and looks at the retail trends for 2015 as well as retailers creatively utilizing innovations to address the disruptive changes currently impacting the marketplace. It also provides a look at the world's 50 largest e-retailers, an outlook for the global economy, and an analysis of market capitalization in the retail industry.}

Retail trends for 2015 are shaped by the disruptive changes currently impacting the marketplace. Here we focus on five main trends. The first is travel retailing, which is redefining notions of customer base and transforming the role airport retail plays in a company's strategy. The second is mobile retailing, a rapidly growing business that is expected to approach US\$640 billion in annual global sales within just a few years. The third is faster retailing, which is just what the name implies: speed to market, speed to response, speed to deliver. The fourth is experience retailing, which takes shopping and adds entertainment, emotion, deeper engagement, and sometimes even an entire environment. And finally there is innovative retailing, which responds to market disruption with disruptive creativity of its own.

While these trends are not new, what is interesting for 2015 is that even though the advances in technologies available to consumers and retailers have been exponential, consumers and retailers are more and more willing and quicker to experiment, adopt and move on to the latest technologies in more creative and innovative ways. Some retailers are even keeping up with the neck-breaking speed of change, leveraging innovation and big data to their advantage; rethinking their business model and adapting every aspect of their operations; executing marketing, product and service on every channel necessary; and responding $24 / 7$ to $24 / 7$ consumers with increasing expectations.

With market disruption comes opportunities, and obviously, the retailers who can be nimble, adapt, and innovate in the face of these changes will be in a better position for success than those who cannot.

\section{Travel retailing}

Over a billion people travel internationally each year approximately 15 percent of the global population - and they spend the equivalent of more than a trillion US dollars in the process.' As a result, companies such as Pernod Ricard and L'Oréal refer to travel retail as their "sixth continent," and Luxottica describes airport sales as "the Formula One of retail."

International tourism continues to rise above expectations despite continuing global geopolitical and economic challenges, and sales have grown by more than 12 percent a year since $2009 .^{2}$ Half of that growth has come from an increase in travelers, especially from emerging countries like China. Much of the rest is due to the willingness of travelers to shop en route and abroad (and retailers' improved capability to serve them).

The expanding middle classes of emerging markets are traveling to the world's capitals and boosting sales, especially of luxury products, and this is benefiting the developed economies of the U.S. and Europe. Over half of France's 16 billion Euros luxury industry depends on tourists. ${ }^{3}$ Therefore, in 2015, expect retailers to continue catering to high-spending travelers, especially emerging market tourists, to drive growth.

\footnotetext{
1 Travel Retail: A Sixth Continent with 1 Billion Consumers, Luxury Society, 24 June 2014. http://luxurysociety.com/articles/2014/06/travel-retail-a-sixthcontinent-with-1-billion-consumersinside-frances-168-billion-luxury-goodsmarket

2 Airport shopping: The sixth continent, The Economist, 10 May 2014. http:// www.economist.com/news/business/21601885-battle-catch-people-goldenhour-they-board-getting-ever-more

3 Inside France's €16.8 Billion Luxury Goods Market, Luxury Society, 13 February 2014. http://luxurysociety.com/articles/2014/02/ inside-frances-168-billion-luxury-goods-market
} 
Travel retail provides new opportunities to engage with consumers. Travelers often have time for leisurely shopping due to lengthy wait times at airports, and international travel often promotes a sense of personal achievement, both conditions providing a good atmosphere for experimentation and indulgence.

In response to these opportunities many companies are investing in building brand awareness in emerging countries, even when the targeted consumers may not purchase those brands at home. This is because these consumers are keen to acquire foreign and luxury brands while travelling, especially in developed markets which offer superior product selection and availability as well as advantageous price comparisons due to high import taxes in home countries.

As a result, airports have become retail destinations and airport retail design has evolved, now dominated by luxury fashion trends rather than the duty free shops of the past. Airports have also become laboratories and an important source of data, allowing companies to experiment. For example, World Duty Free Group (WDFG), Heathrow Airport's "anchor" retailer, uses airport data to better prepare for international arrivals, such as ensuring that speakers of the right languages and cultural sensitivities will be on hand. They can even reconfigure shop displays to suit the national tastes of travelers passing through.

\section{Mobile retailing}

A projected 65 percent of the global population will be using a mobile phone by 2015 and an estimated 83 percent of internet usage will be through handheld devices. ${ }^{4}$ It is no surprise then that mobile retailing is expected to continue to grow aggressively. In the next three years, global e-commerce sales made via mobile devices are expected to top US\$638 billion, which is about the size of the world's entire e-commerce market just a year ago. ${ }^{5}$

The introduction of wearables, such as Google Glass and Apple Watch, opens new opportunities for reaching consumers, and retailers will keep an eye on developments in this arena. The smart watch sector is currently estimated at US\$1.4 billion to US\$1.8 billion but is expected to surge to US\$10 billion by $2018 .{ }^{6}$ The full range of the wearable technology market is projected to hit US\$30 billion in sales during the same period. Some predictions have shoppers purchasing via wearable devices as early as 2015 .

Retailers will need to respond by offering free in-store Wi-Fi and mobile-friendly retail websites optimized for different kinds of personal devices. Mobile payments will play a large role - Forrester expects mobile payments to amount to US\$90 billion by 2017 as will location-based marketing. Wal-Mart, eBay and Amazon have already created convenient mobile customer experiences and others will follow. However, while shoppers want real-time, relevant, and personalized information and offers, retailers will need to surround this service with very strong privacy and security. Trust, transparency, and protecting customer information will be critical in retaining loyalty as mobile retailing becomes the norm.

\section{Faster retailing}

Speed has been an important trend in retail for over a decade. This includes: "fast fashion" (getting runway styles to the stores as soon as possible); limited-time-only products and flash sales to drive urgency and immediate purchase; pop-up establishments to quickly get products and services to market and build buzz; and self-service check-out and kiosks to reduce or eliminate waiting.

In 2015, expect retailing to get even faster to meet consumers' desires. Millennials will be driving much of this as they are the largest generation, with a lot of spending power, and carry a lot of influence. They prefer fast response and immediate gratification, and retailers will cater to that. With Amazon and Google offering same-day delivery in certain areas, expect other companies to follow; the delivery window will become more narrow and specific.

This won't happen overnight, of course, but the first-movers in this area are aggressively testing possibilities. Amazon expanded its same-day delivery service in parts of the U.S. to certain cities in the UK, Germany, and Canada. It recently tested delivery by taxi, and is waiting for FAA permission to begin their Prime Air service in which drones will deliver packages within 30 minutes. The ability to meet the consumer's need for speed makes an efficient end-to-end supply chain more important than ever.

While same-day product delivery will not likely be the norm for everyone, instant availability of information will be. More and more consumers are expecting it and retailers will need to deliver. Retailers will need to optimize their information to provide as much content as shoppers need without the load time, especially over Wi-Fi and cell networks on mobile devices, so shoppers can easily and quickly find the information or product they need.

4 Two Billion Smartphone Users By 2015: 83\% of Internet Usage From Mobiles, Dazelnfo, 23 January 2014. http://www.dazeinfo.com/2014/01/23/ smartphone-users-growth-mobile-internet-2014-2017/

5 US E-Commerce Growth Is Now Far Outpacing Overall Retail Sales, Business Insider, 2 April 2014. http://www.businessinsider.com/ us-e-commerce-growth-is-now-far-outpacing-overall-retail-sales-2014-4

6 Apple Smartwatch Launch Boost to Wearables, Women's Wear Daily, 10 October 2014. http://www.wwd.com/media-news/digital/ apple-unveils-smartwatch-7890678?src=n/newsAlert/20140909-3 


\section{Experience retailing}

Retailing is no longer just about the product, but the experience. Consumers want shopping to include entertainment, education, emotion, engagement, and enlightenment. Retailers are exploring innovative ways to enhance the buying experience for their customers: fashion shows, music festivals, tablet and interactive displays, social media campaigns, and product and marketing co-creation. The Legaspi Company has even rebuilt failing shopping malls into Hispanic cultural centers serving all the needs of a family: grocery stores, dental and medical care, clothes, entertainment, banking, a department of motor vehicles, and spaces for religious activities; and seen income and foot traffic increase at these locations, typically by 30 percent.

It should be noted that with all these new forms of engagement, regardless of in-store, online, at home, or on the street, consumers still want a seamless and consistent experience. They expect to be able to view online information, access coupons, learn about promotions, and review inventory at any time on any device. They expect messaging and product and pricing information to be consistent across all channels: advertising and social media; in-store and online; pushed or pulled; the sales people on the floor as well as the customer service representative on the phone. They want to order, pick up, ship, receive, and return anywhere. To become nimble enough to respond to these consumer expectations, some retailers may have to change their internal operations, removing silos and improving cross-functional collaboration.

Personalized websites and emails have become the norm, and consumers are looking for this personalization to extend into their in-store experiences. Retailers can now send customized text messages to shoppers in-store, and adapt interactions to each individual. Retailers will therefore be investing in analysis of big data to enable personalization. However, trust will be critical since violation of privacy will be a concern for consumers. Retailers will need to be transparent in their collection and use of data, and educate shoppers about the value they are delivering with personalized shopping experience.
Innovative retailing

In 2015, the retail industry will continue to be disrupted by new technologies and innovative competition. There will be no single formula for success, which will come in all shapes and sizes, formats and channels. We will continue to see the blurring of sectors as well as single product retailing, such as Warby Parker. There will continue to be non-traditional retailers innovating in the retail space, such as Verizon with its Mall of America destination store. Mobile POS systems will continue to enable inventive pop-up stores, trucks, and kiosks. Retailers will be vertically integrated and vertically transparent, such as Zady. In some cases the middleman will be cut out completely, with no physical stores, no inventory, no warehouses, but rather made-to-order direct from manufacturer, such as Awl \& Sundry and Made.com. The line between retailer and producer will begin to blur in new ways, such as Amazon's Creative Expressions, part of their 3-D Printing Store.

Expect more retailers to be innovators. Numerous retailers have invested heavily in their own innovation labs, including Nordstrom, Wal-Mart, Staples, Amazon, and The Home Depot. Retailers are embracing technology and using it in creative ways. For example, Lowe's has introduced a multi-lingual robot that can scan a part a shopper brings in, identify it, instantly access information about it, and help the customer find it in the store. With the internet of things, we can soon expect retail innovations like automatic purchasing and delivery with connected homes and refrigerators.

The near future of the retail industry is about adaptation and embracing change. The speed of innovation and the disruption it causes won't cease, and the demands of customers will continue to escalate. To thrive in this environment retailers will respond quickly to threats and opportunities with innovations of their own. This requires connecting strategy, capabilities, and specific initiatives, guided by the insights provided by market data.

The right talent with the right skill set is key to successful execution, of course. Retailers will need to focus on finding, recruiting, and retaining the best people. But the reality is that no retailer will have all the appropriate talent in-house, making it essential that they develop an arrangement of partnerships and specialized resources. So when needed, they can quickly call upon the right expertise to drive the kind of innovation in product offerings, business models, and customer engagement that will enable them to stay ahead of the competition. 


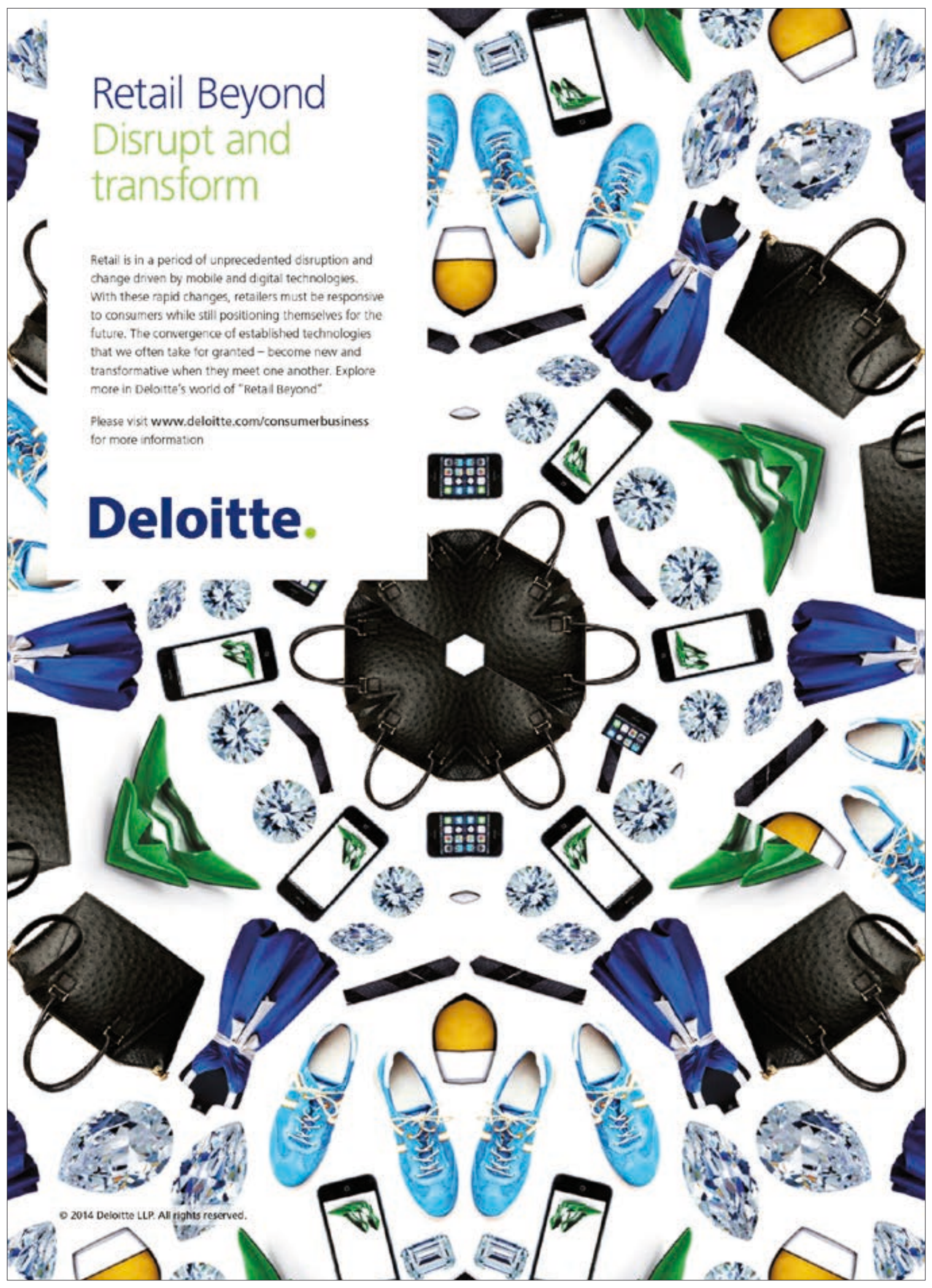




\section{Global economic outlook}

The global economy appears to be decelerating as several large economies face increasing trouble. The main ones of concern are China and the Eurozone as well as a few key emerging markets such as Brazil and Russia. China has experienced decelerating growth as it struggles to balance the need to avoid excessive credit growth with a desire to keep the economy moving. In Europe, weakness in credit markets is preventing economic growth from resuming at a normal pace.

On the other hand, the brightest spots in the global panorama are the US and British economies. The US appears to be on a self-sustaining path of faster growth combined with low inflation. One factor driving that growth is the huge increase in oil production which, in turn, has led to lower energy prices globally. The low energy prices are a problem for oil exporters such as Russia and Venezuela, while this is benefitting oil consumers such as India and Japan.

Among the big issues having a global impact are the following:

\section{The shift in US monetary policy}

The Federal Reserve has ended its program of asset purchases and is now assessing when to raise short term interest rates for the first time in eight years. This transition, and the expectation of higher rates, is driving up the value of the US dollar and putting downward pressure on other currencies. That, in turn, has forced emerging market central banks to raise interest rates, thus slowing the growth of these economies. That slower growth is evident in such diverse places as Brazil, Turkey, Indonesia, South Africa, Russia, and Argentina. Once US monetary policy returns to normal, emerging countries will be better able to shift toward stable interest rates.

\section{Energy production in the US}

This has risen precipitously due to the new technology of horizontal hydraulic fracturing of shale rock - better known simply as fracking. The rise in US energy output has lowered global energy prices, reduced the US trade deficit, boosted US economic growth, improved the competitiveness of energy-intensive US manufacturers, and suppressed global inflation - especially in developed economies. It has also hurt competing energy producers such as Russia, Venezuela, and Nigeria. From a retail perspective, lower energy prices have added to the purchasing power of consumers in energy consuming countries - thus providing a boost for retailers.
The crisis in Ukraine

The conflict between Russia and the West over Ukraine, which has resulted in punitive sanctions imposed on Russia by the EU and the US, is hurting the Russian economy and, by extension, the Eurozone economy. Fear of further sanctions is having a chilling effect on investment in Russia as well as in Germany. The German economy has slowed significantly, in part, due to problems in Russia. About 6,000 German companies have investments in Russia and are dependent on sales of their products and services in Russia. Each time the crisis worsens there is a flight to safety which results in a stronger US dollar and lower US bond yields.

\section{A slowdown in Chinese investment}

The deceleration of the Chinese economy, which has involved a slowdown in the growth of fixed asset investment, has led to a sharp drop in global commodity prices. This has hurt such countries as Australia, Brazil, Indonesia, and Chile. Plus, China's financial system is at risk due to a sharp rise in shadow banking debt, thus boding poorly for growth if a financial crisis emerges. For the world's leading commodity producers, this suggests a need to focus on other sources of growth in the future.

\section{Demographics}

A slowing of labor force growth and an increase in the retired populations of the US, Japan, Europe, and China are all playing a role in reducing growth prospects. In addition, these demographic trends are putting pressure on governments which must spend more on pensions and healthcare. Different countries are approaching the problem in different ways. In China, the government is gradually lifting the restrictions on numbers of children and, in the process, promoting a higher birth rate. In Japan, the government is encouraging greater female participation in the labor force. In Europe and the US, a debate rages as to the degree to which immigration should be allowed and encouraged. Supporters of immigration see it as a vehicle for boosting the ratio of workers to retirees and, in the process, alleviating pressure on pensions. In addition, immigration is a tool for boosting the rate of economic growth. Meanwhile, those countries with youthful demographics are poised for relatively strong economic growth. These include India, much of Latin America, and much of the Middle East and Africa.

The ubiquity of the internet

This has already significantly altered countless industries such as bookselling, music, travel, insurance, banking, entertainment, and even tax preparation and consulting. The effect is to put downward pressure on the prices of everything, eliminate countless mid-level jobs, increase demand for highly skilled workers, and exacerbate income inequality. Also, the internet is leading more people to work and shop from home, thus reducing demand for cars, energy, and commercial property. 
What follows is a closer look at the world's major economies and the implications for the retailing industry:

\section{United States}

The US economy has been doing very well. Most indicators now point to the likelihood of strong growth in 2015 and possibly beyond. Industrial output, employment, and retail sales have been performing well. Even housing, which had shown signs of trouble earlier in 2014, has lately staged a modest comeback. The end of aggressive monetary policy (QE) is being absorbed well by the economy. The only uncertainty concerns when the Federal Reserve will eventually raise short term interest rates - something it hasn't done in eight years. As of late 2014, inflation remains very low and the labor market continues to exhibit slack as evidenced by weak wage growth, thus possibly extending the period of low interest rates. The Federal Reserve is tasked with balancing two goals: low inflation and low unemployment. A decision about the timing of an increase in short term interest rates will depend on where these indicators stand and, perhaps more importantly, where expectations of inflation stand.

Consumers, having substantially paid off debts, are spending at a brisk pace - even though wages have been relatively dormant. Businesses have hoarded cash and engaged in a flurry of stock buybacks. Yet there is reason to expect a boost to investment in the coming year given rising demand. Despite weak overseas growth, exports are performing reasonably well due to increased competiveness, especially on the back of low domestic gas prices. Fiscal policy is essentially neutral and is not likely to change much for the remainder of the current presidency.

On the other hand, income inequality continues to increase, thus hurting consumer spending because only a small share of consumers are experiencing significant income gains. The failure of the government to enact immigration reform means that the country continues to face a shortage of skilled workers, especially in the realm of information technology. Finally, there is a risk that some members of Congress will, once again, make raising the debt ceiling a contentious issue. If this happens, it could have a temporary negative impact on asset prices, business investment, and hiring.

\section{Europe}

The Eurozone is in trouble. After a promising start to the year, the region's economy has decelerated quickly, led by Germany traditionally the engine of European growth. Also France is weak and Italy is back in recession. Among major Eurozone economies, only Spain is experiencing a significant rebound in growth although unemployment remains ruinously high. The region suffers from several problems:

- First, credit markets continue to fail. Despite an increasing money supply, bank lending is declining. This reflects the fact that many banks in the region remain laden with bad debts and are attempting to recapitalize by cutting lending and selling risky assets. This is especially problematic in southern Europe where, as a result of perceived banking problems, risk spreads remain high. This discourages borrowing. Plus, the fear of deflation is discouraging businesses from borrowing for the purpose of investment.

- Second, fiscal austerity has had a negative impact on growth. In Germany, where there is now a balanced budget, the government insists on further cuts in spending in order to produce a surplus. Some critics, including the IMF, US government, ECB, and many countries within the Eurozone, have called on Germany to borrow cheaply in order to boost spending on infrastructure. The idea is that such spending would boost domestic demand in both Germany and the rest of Europe. Yet Germany insists that it will not revert to fiscal stimulus.

- Third, many countries, such as France and Italy, remain mired in onerous regulations - especially of their labor markets. This depresses productivity, elevates unemployment, and hurts exports. While both governments intend to reform their labor laws, the pace of reform has been modest.

- Fourth, the Russian/Ukraine crisis has had a negative impact on Europe's economies, especially those of Germany, Finland, Poland, and the Baltics - all of which have significant economic relations with Russia.

The only major effort to reverse Europe's dilemma comes from the European Central Bank (ECB), which has implemented a more aggressive monetary policy involving low interest rates, direct loans to banks, and purchases of securitized assets. Some critics fear that the program will not be sufficient. Moreover, it is not clear that the ECB will be willing to go further and purchase government bonds given substantial German opposition. Initially, the take-up on the lending program was weak given that banks are not confident that there would be sufficient demand for cheap loans. Hence, the outlook for the Eurozone is modest at best. There remains a significant risk of a regional recession. 
There are a few bright spots in Europe: Britain, Ireland, and Spain. The British economy is growing rapidly following an aggressive monetary policy and government subsidies for housing. On the other hand, there is increasing concern that Britain may ultimately exit the EU. In Ireland, growth has resumed at a strong pace as the economy recovers from a very deep recession. The Spanish economy is rebounding nicely following productivity gains and wage restraint, both of which have buttressed export competitiveness. Yet Spain faces very high unemployment as well as a separatist movement in Catalonia.

\section{China}

China's economy has slowed down and continues to show signs of weakness despite government efforts to reverse the slowdown. Growth is now slightly above 7.0 percent which, if sustained, would be the slowest pace of growth in nearly two decades. While this seems high in comparison to developed economies, it is low by recent historical standards in China. Moreover, growth below this level would mean an inability to absorb workers migrating from rural to urban areas. The result would be high unemployment and social unrest. And, if the workers didn't migrate, China wouldn't grow since there would be zero productivity gains that come from switching workers from farms to factories. Thus, China cannot afford to grow any slower. Why is China decelerating?

- First, export markets such as Europe have been dormant. Even the US market isn't what it used to be for China. Plus, China's wages and currency have increased, thereby reducing the competitiveness of Chinese exports. The result has been that some manufacturing capacity has moved outside of China. Companies are looking elsewhere to produce goods for export. Basic assembly is moving from China to Vietnam, Indonesia, and elsewhere.

- Second, the government has attempted to limit the growth of the shadow banking system. Lending outside traditional banking channels has resulted in excess investment in property, infrastructure, and heavy industry. The result is a growing volume of non-performing assets which threatens the stability of the financial system. Yet efforts to limit this activity have contributed to the slowdown in growth. The government is torn between a desire to limit financial risk and a desire to avoid a sharp slowdown. It has taken measures to limit the growth of shadow banking while, at the same time, attempted to stimulate more traditional forms of credit. Yet it has so far failed to liberalize the financial system.

- Third, a modest fiscal stimulus implemented in 2014 appears to be unwinding, thus removing stimulation for the economy.

- Fourth, a government crackdown on corruption has had a chilling effect on high end retail spending as well as on some areas of business investment.
Going forward, China will require significant reforms if it is to retain strong growth. The government has proposed such reforms, but has not acted. These would entail liberalizing financial services, improving management of state-run companies, creating a level playing field for private companies, protecting property rights, boosting the social safety net for consumers in order to stimulate more spending, and investing more in education and public health. For now, the outlook for reform is murky as the leadership struggles to choose between short term and long term goals.

Japan

Under Prime Minister Shinzo Abe, Japan has engaged in a very aggressive monetary policy involving massive purchases of assets - similar to the quantitative easing undertaken by the US Federal Reserve. In late 2014, the Bank of Japan increased the pace of asset purchases in an effort to boost expectations for higher inflation. The policy boosted inflation, suppressed the value of the yen, increased equity prices, and reduced real borrowing costs. Yet although the policy initially boosted economic activity in early 2013, it has since abated. Moreover, a major tax increase in April 2014 caused a rapid drop in economic activity from which Japan is still reeling. Another scheduled tax increase in 2015 threatens to undermine recovery and, as of this writing, it is not clear if the government will go through with the second tax increase. Wages are not rising in line with inflation, which means that real consumer purchasing power is declining. And businesses are reluctant to invest until it is clear that inflation is here to stay. They fear that a return to deflation will reduce the return on any investment. Thus, the growth outlook is uncertain. Many businesses are hoping that Prime Minister Abe will implement the deregulatory component of "Abenomics." But Abe has been reluctant to act on issues that are politically difficult.

The one issue on which the Prime Minister has been quite aggressive is his effort to boost female participation in the labor force. In Japan, female participation is considerably lower than in other developed economies. Abe believes that, if participation increases, economic growth will accelerate during the period that participation increases. In addition, increased participation would alleviate pension difficulties. From a retail perspective, increased female participation would probably lead to increased modernization and consolidation of retailing. 


\section{Other emerging markets}

\section{Brazil}

Brazil's economy is in recession. This is due to a sharp drop in commodity prices (due to China's slowdown); a central bank policy of high interest rates to fight inflation and to stabilize the currency; and business lack of confidence due to a government policy of high regulation and protectionism. The election in late 2014 resulted in a second term for President Dilma Rousseff. As of this writing, it is not clear if she will shift toward the center and endorse more market oriented policies that the business community has urged. Her major platform has been an effort to boost the spending power of the poor through government transfers.

The economic outlook is poor as there is likely to be continued downward pressure on the currency due to the impending shift in US monetary policy. Thus, the central bank will likely be compelled to keep interest rates high. In addition, Brazilian domestic demand is likely to be constrained by the very high level of consumer debt relative to disposable income. Exports of commodities are likely to be restrained due the slowdown in China and manufactured exports lack competitiveness.

\section{India}

India had a highly significant election in 2014, resulting in the election of Narendra Modi as Prime Minister. For the first time in more than 30 years, a Prime Minister's party has a majority in the Parliament, thus boosting prospects for enacting reform legislation. Yet in his first six months in office, not much reform legislation was proposed, thus disappointing supporters who were euphoric following Modi's victory. They are hoping he will deregulate industry and labor markets, reduce costly subsidies, boost infrastructure investment, negotiate freer trade, and ease restrictions on foreign investment. If he does these things, India's growth outlook will likely improve dramatically. Meanwhile, growth is recovering from its doldrums but remains below potential. The central bank has managed to reduce inflationary expectations, which should have a positive impact on growth.

\section{Russia}

Russia's economy is in bad shape. Following the crisis in Ukraine and the implementation of sanctions, there was massive capital flight from Russia resulting in a sharp drop in the currency. This, in turn, led the central bank to severely raise interest rates several times. The result has been that investment dried up, including foreign investment. Moreover, the declining global price of oil has also contributed to downward pressure on the currency and concerns about the ability of Russian debtors to service their external debts. The sanctions that have been imposed mean that some big Russian companies will have trouble rolling over foreign debts due to limited access to foreign financial markets. Some energy companies will lack access to technologies that are needed to tap into Arctic reserves. This means that oil production is likely to decline absent an end to sanctions.
To deal with the deteriorating economic relations between Russia and the West, the Russian government is attempting to pivot toward China as a source of capital and as an export market. Yet this is unlikely to be an adequate replacement to Europe. Longer term, Russia faces competition as the US is likely to export more oil and liquid natural gas to Europe. Thus, the outlook for Russia is uncertain.

\section{Mexico}

Mexico's economy is not currently growing strongly. Yet that hasn't stopped investors from bursting with enthusiasm. There are two major reasons. First, Mexico's reformist government has liberalized foreign investment in energy, deregulated telecoms and media, and attempted to end public corruption. Second, Mexico is experiencing a renaissance in manufacturing due to higher wages in China (and therefore more competitive labor costs in Mexico), lower energy prices in North America, and a rebound in US demand. Thus, the outlook for Mexico is quite good.

\section{Africa}

Sub-saharan Africa is the second fastest growing economic region of the world after East Asia. This is due to a confluence of positive factors including substantial inbound investment in commodity production, strong commodity exports, better governance in several countries, an end to civil conflict in a number of countries, youthful demographics that have boosted the labor force, and increased domestic demand in Africa's largest economies. Although commodity prices have come down, Africa's growth prospects still remain positive. The rising middle class is contributing to the modernization of retailing and greater consumer market opportunities. Moreover, Africa has become a surprising laboratory for experimentation in mobile commerce. 


\section{Top 250 global retailers 2013}

Top 250 global retailers, 2013

\begin{tabular}{|c|c|c|c|c|c|c|c|c|}
\hline $\begin{array}{l}\text { Retail } \\
\text { revenue } \\
\text { rank } \\
\text { (FY13) }\end{array}$ & Name of company & $\begin{array}{l}\text { Country } \\
\text { of origin }\end{array}$ & $\begin{array}{r}2013 \\
\text { net retail } \\
\text { revenue } \\
(\text { US\$m) }\end{array}$ & $\begin{array}{r}2013 \\
\text { parent } \\
\text { company/ } \\
\text { group } \\
\text { revenue } \\
\text { (US\$m) }\end{array}$ & $\begin{array}{r}2013 \\
\text { parent } \\
\text { company/ } \\
\text { group } \\
\text { net income } \\
(\text { US\$m) }\end{array}$ & Dominant operational format 2013 & $\begin{array}{r}\# \\
\text { countries } \\
\text { of } \\
\text { operation } \\
2013\end{array}$ & $\begin{array}{r}2008- \\
2013 \\
\text { retail } \\
\text { revenue } \\
\text { CAGR }^{2}\end{array}$ \\
\hline 1 & Wal-Mart Stores, Inc. & U.S. & 476,294 & 476,294 & 16,695 & Hypermarket/Supercenter/Superstore & 28 & $3.3 \%$ \\
\hline 2 & Costco Wholesale Corporation & U.S. & 105,156 & 105,156 & 2,061 & Cash \& Carry/Warehouse Club & 9 & $7.7 \%$ \\
\hline 3 & Carrefour S.A. & France & 98,688 & 101,844 & 1,812 & Hypermarket/Supercenter/Superstore & 33 & $-3.0 \%$ \\
\hline 4 & Schwarz Unternehmens Treuhand KG & Germany & $98,662^{\mathrm{e}}$ & $98,662^{\mathrm{e}}$ & $\mathrm{n} / \mathrm{a}$ & Discount Store & 26 & $6.5 \%$ \\
\hline 5 & Tesco PLC & U.K. & 98,631 & 100,213 & 1,529 & Hypermarket/Supercenter/Superstore & 13 & $2.9 \%$ \\
\hline 6 & The Kroger Co. & U.S. & 98,375 & 98,375 & 1,531 & Supermarket & 1 & $5.3 \%$ \\
\hline 7 & Metro $\mathrm{Ag}^{3}$ & Germany & $86,393^{\mathrm{e}}$ & $86,393^{e}$ & $588^{e}$ & Cash \& Carry/Warehouse Club & 32 & $-0.9 \%$ \\
\hline 8 & Aldi Einkauf GmbH \& Co. oHG & Germany & $81,090^{e}$ & $81,090^{\mathrm{e}}$ & $\mathrm{n} / \mathrm{a}$ & Discount Store & 17 & $5.5 \%$ \\
\hline 9 & The Home Depot, Inc. & U.S. & 78,812 & 78,812 & 5,385 & Home Improvement & 4 & $2.0 \%$ \\
\hline 10 & Target Corporation & U.S. & 72,596 & 72,596 & 1,971 & Discount Department Store & 2 & $2.9 \%$ \\
\hline 11 & Walgreen Co. & U.S. & 72,217 & 72,217 & 2,450 & Drug Store/Pharmacy & 2 & $4.1 \%$ \\
\hline 12 & CVS Caremark Corporation & U.S. & 65,618 & 126,761 & 4,592 & Drug Store/Pharmacy & 3 & $6.0 \%$ \\
\hline 13 & Casino Guichard-Perrachon S.A. & France & $63,468^{\star *}$ & $64,613^{* *}$ & 2,023 & Hypermarket/Supercenter/Superstore & 29 & $11.1 \%$ \\
\hline 14 & Groupe Auchan SA & France & 62,444 & 63,859 & 1,109 & Hypermarket/Supercenter/Superstore & 13 & $4.0 \%$ \\
\hline 15 & Amazon.com, Inc. & U.S. & 60,903 & 74,452 & 274 & Non-Store & 14 & $26.7 \%$ \\
\hline 16 & Edeka Zentrale AG \& Co. KG & Germany & $59,704^{* *}$ & $61,399^{* *}$ & $\mathrm{n} / \mathrm{a}$ & Supermarket & 1 & $5.9 \%$ \\
\hline 17 & Aeon Co., Ltd. & Japan & $57,986^{\star *}$ & $64,271^{* *}$ & 835 & Hypermarket/Supercenter/Superstore & 10 & $3.9 \%$ \\
\hline 18 & Woolworths Limited & Australia & 54,457 & 55,974 & 2,258 & Supermarket & 2 & $4.3 \%$ \\
\hline 19 & Seven \& i Holdings Co., Ltd. & Japan & $54,258^{* *}$ & $56,600^{* *}$ & 1,890 & Convenience/Forecourt Store & 18 & $-0.1 \%$ \\
\hline 20 & Lowe's Companies, Inc. & U.S. & 53,417 & 53,417 & 2,286 & Home Improvement & 4 & $2.1 \%$ \\
\hline 21 & Rewe Combine & Germany & $51,109^{* *}$ & $55,745^{* *}$ & 266 & Supermarket & 11 & $3.5 \%$ \\
\hline 22 & Wesfarmers Limited & Australia & 50,711 & 55,265 & 2,076 & Supermarket & 2 & $4.8 \%$ \\
\hline 23 & Centres Distributeurs E. Leclerc & France & $47,671^{\mathrm{e}^{* *}}$ & $60,5699^{* *}$ & $\mathrm{n} / \mathrm{a}$ & Hypermarket/Supercenter/Superstore & 7 & $5.4 \%$ \\
\hline 24 & Koninklijke Ahold N.V. & Netherlands & $43,321^{* *}$ & $43,321^{* *}$ & 3,370 & Supermarket & 7 & $4.9 \%$ \\
\hline 25 & Best Buy Co., Inc. & U.S. & 42,410 & 42,410 & 523 & Electronics Specialty & 5 & $-1.2 \%$ \\
\hline 26 & J Sainsbury plc & U.K. & 38,031 & 38,076 & 1,138 & Supermarket & 1 & $4.8 \%$ \\
\hline 27 & $\begin{array}{l}\text { ITM Développement International } \\
\text { (Intermarché) }\end{array}$ & France & $37,351^{\mathrm{e}^{\star \star *}}$ & $52,998^{9^{* *}}$ & $\mathrm{n} / \mathrm{a}$ & Supermarket & 6 & $2.0 \%$ \\
\hline 28 & The IKEA Group (INGKA Holding B.V.) & Netherlands & 36,495 & 37,288 & 4,339 & Other Specialty & 43 & $5.6 \%$ \\
\hline 29 & Sears Holdings Corp. & U.S. & 36,188 & 36,188 & $-1,116$ & Department Store & 3 & $-5.0 \%$ \\
\hline 30 & Safeway Inc. & U.S. & 35,011 & 36,139 & 3,522 & Supermarket & 3 & $-4.1 \%$ \\
\hline 31 & Loblaw Companies Limited & Canada & $30,697^{\star *}$ & $31,446^{* *}$ & 612 & Hypermarket/Supercenter/Superstore & 2 & $0.5 \%$ \\
\hline 32 & Publix Super Markets, Inc. & U.S. & 29,148 & 29,148 & 1,654 & Supermarket & 1 & $3.9 \%$ \\
\hline 33 & Delhaize Group SA & Belgium & $28,037^{\star *}$ & $28,037^{* *}$ & 243 & Supermarket & 9 & $2.1 \%$ \\
\hline 34 & Macy's, Inc. & U.S. & $27,931^{* *}$ & $27,931^{* *}$ & 1,486 & Department Store & 3 & $2.3 \%$ \\
\hline 35 & Wm Morrison Supermarkets PLC & U.K. & 27,739 & 27,739 & -373 & Supermarket & 1 & $4.0 \%$ \\
\hline 36 & The TJX Companies, Inc. & U.S. & 27,423 & 27,423 & 2,137 & Apparel/Footwear Specialty & 7 & $7.6 \%$ \\
\hline 37 & Rite Aid Corporation & U.S. & 25,526 & 25,526 & 249 & Drug Store/Pharmacy & 1 & $-0.6 \%$ \\
\hline
\end{tabular}

${ }^{1}$ Revenue and net income for the parent company or group may include results from non-retail operations

2 Compound annual growth rate

${ }^{3}$ Metro changed its fiscal year from end of December to end of September. Fiscal 2013 results reported here include the 9 months ended 30 September 2013 plus the quarter ended 31 December 2013 to create a 12-month period equivalent to prior years. e = estimate

$\mathrm{g}=$ gross turnover as reported by company

n/a = not available

ne $=$ not in existence (created by merger or divestiture)

* Revenue reflects wholesale sales

** Revenue includes wholesale and retail sales 


\begin{tabular}{|c|c|c|c|c|c|c|c|c|}
\hline $\begin{array}{l}\text { Retail } \\
\text { revenue } \\
\text { rank } \\
\text { (FY13) }\end{array}$ & Name of company & $\begin{array}{l}\text { Country } \\
\text { of origin }\end{array}$ & $\begin{array}{r}2013 \\
\text { net retail } \\
\text { revenue } \\
(\text { US\$m) }\end{array}$ & $\begin{array}{r}2013 \\
\text { parent } \\
\text { company/ } \\
\text { group } \\
\text { revenue }^{1} \\
\text { (US\$m) }\end{array}$ & $\begin{array}{r}2013 \\
\text { parent } \\
\text { company/ } \\
\text { group } \\
\text { net income }{ }^{1} \\
(\text { US\$m) }\end{array}$ & Dominant operational format 2013 & $\begin{array}{r}\# \\
\text { countries } \\
\text { of } \\
\text { operation } \\
2013\end{array}$ & $\begin{array}{r}2008- \\
2013 \\
\text { retail } \\
\text { revenue } \\
\text { CAGR }^{2}\end{array}$ \\
\hline 38 & Migros-Genossenschafts Bund & Switzerland & $25,010^{\mathrm{e}^{* *}}$ & $28,863^{* *}$ & 847 & Hypermarket/Supercenter/Superstore & 3 & $1.4 \%$ \\
\hline 39 & Système U, Centrale Nationale & France & $24,706^{\mathrm{e}^{\star *}}$ & $31,413^{9^{* *}}$ & $\mathrm{n} / \mathrm{a}$ & Supermarket & 5 & $5.4 \%$ \\
\hline 40 & Lotte Shopping Co., Ltd. & S. Korea & 24,601 & 25,955 & 810 & Hypermarket/Supercenter/Superstore & 6 & $17.5 \%$ \\
\hline 41 & $\begin{array}{l}\text { LVMH Moët Hennessy- } \\
\text { Louis Vuitton S.A. }\end{array}$ & France & $24,392^{\mathrm{e}}$ & $38,717^{* *}$ & 5,243 & Other Specialty & 76 & $14.6 \%$ \\
\hline 42 & Mercadona, S.A. & Spain & 23,954 & 23,954 & 684 & Supermarket & 1 & $4.8 \%$ \\
\hline 43 & Albertson's LLC & U.S. & $23,000^{e}$ & $23,000^{e}$ & $\mathrm{n} / \mathrm{a}$ & Supermarket & 1 & $38.6 \%$ \\
\hline 44 & Inditex, S.A. & Spain & $22,265^{\star *}$ & $22,265^{* *}$ & 3,171 & Apparel/Footwear Specialty & 89 & $10.0 \%$ \\
\hline 45 & H.E. Butt Grocery Company & U.S. & $20,330^{e}$ & $20,330^{\mathrm{e}}$ & $n / a$ & Supermarket & 2 & $6.3 \%$ \\
\hline 46 & Apple Inc./Apple Retail Stores & U.S. & 20,228 & 170,910 & 37,037 & Electronics Specialty & 14 & $22.6 \%$ \\
\hline 47 & Cencosud S.A. & Chile & 19,855 & 20,889 & 505 & Supermarket & 5 & $11.3 \%$ \\
\hline 48 & Empire Company Limited & Canada & $19,829^{* *}$ & $19,890^{* *}$ & 230 & Supermarket & 1 & $7.3 \%$ \\
\hline 49 & H \& M Hennes \& Mauritz AB & Sweden & 19,729 & 19,729 & 2,632 & Apparel/Footwear Specialty & 54 & $7.7 \%$ \\
\hline 50 & Coop Group & Switzerland & 19,529 & $29,111^{* *}$ & 592 & Supermarket & 1 & $1.1 \%$ \\
\hline 51 & A.S. Watson Group & $\begin{array}{l}\text { Hong Kong } \\
\text { SAR }\end{array}$ & $19,230^{\star *}$ & $19,230^{* *}$ & $\mathrm{n} / \mathrm{a}$ & Drug Store/Pharmacy & 25 & $4.7 \%$ \\
\hline 52 & Kohl's Corporation & U.S. & 19,031 & 19,031 & 889 & Department Store & 1 & $3.0 \%$ \\
\hline 53 & Yamada Denki Co., Ltd. & Japan & $18,921^{* *}$ & $18,921^{* *}$ & 199 & Electronics Specialty & 7 & $0.2 \%$ \\
\hline 54 & Groupe Adeo SA & France & $18,197^{\mathrm{e}^{* *}}$ & $18,197^{\mathrm{e}^{* *}}$ & $\mathrm{n} / \mathrm{a}$ & Home Improvement & 13 & $10.0 \%$ \\
\hline 55 & OJSC "Magnit" & Russia & 18,197 & $18,202^{* *}$ & 1,118 & Convenience/Forecourt Store & 1 & $27.9 \%$ \\
\hline 56 & Dollar General Corporation & U.S. & 17,504 & 17,504 & 1,025 & Discount Store & 1 & $10.9 \%$ \\
\hline 57 & Kingfisher plc & U.K. & 17,454 & 17,454 & 1,114 & Home Improvement & 9 & $2.1 \%$ \\
\hline 58 & X5 Retail Group N.V. & Russia & 16,785 & 16,785 & 345 & Discount Store & 2 & $15.0 \%$ \\
\hline 59 & Suning Commerce Group Co., Ltd. & China & 16,616 & 17,010 & 60 & Electronics Specialty & 3 & $16.3 \%$ \\
\hline 60 & Marks and Spencer Group plc & U.K. & 16,391 & 16,391 & 804 & Department Store & 48 & $2.6 \%$ \\
\hline 61 & The Gap, Inc. & U.S. & 16,148 & 16,148 & 1,280 & Apparel/Footwear Specialty & 54 & $2.1 \%$ \\
\hline 62 & Jerónimo Martins, SGPS, S.A. & Portugal & 15,712 & 15,712 & 521 & Discount Store & 3 & $12.3 \%$ \\
\hline 63 & Coop Italia & Italy & $15,211^{\mathrm{e}}$ & $16,901^{\mathrm{g}}$ & $\mathrm{n} / \mathrm{a}$ & Hypermarket/Supercenter/Superstore & 1 & $-0.2 \%$ \\
\hline 64 & Meijer, Inc. & U.S. & $15,000^{\mathrm{e}}$ & $15,000^{\mathrm{e}}$ & $\mathrm{n} / \mathrm{a}$ & Hypermarket/Supercenter/Superstore & 1 & $1.8 \%$ \\
\hline 65 & ICA Gruppen AB & Sweden & $14,952^{* *}$ & $15,274^{* *}$ & 219 & Supermarket & 6 & $1.7 \%$ \\
\hline 66 & El Corte Inglés, S.A. & Spain & 14,789 & 19,055 & 232 & Department Store & 5 & $-4.4 \%$ \\
\hline 67 & $\begin{array}{l}\text { Conad Consorzio Nazionale, } \\
\text { Dettaglianti Soc. Coop. a.r.I. }\end{array}$ & Italy & $14,438^{\mathrm{e}^{* *}}$ & $15,408^{g^{* *}}$ & $\mathrm{n} / \mathrm{a}$ & Supermarket & 2 & $5.8 \%$ \\
\hline 68 & John Lewis Partnership plc & U.K. & $14,164^{* *}$ & $14,164^{* *}$ & 159 & Supermarket & 3 & $7.6 \%$ \\
\hline 69 & Gome Home Appliance Group & China & $13,441^{e}$ & $13,782^{\text {ge }}$ & $\mathrm{n} / \mathrm{a}$ & Electronics Specialty & 1 & $3.2 \%$ \\
\hline 70 & Otto (GmbH \& Co KG) & Germany & 13,355 & 17,100 & 239 & Non-Store & 54 & $2.5 \%$ \\
\hline 71 & S Group & Finland & 13,233 & 15,080 & 300 & Supermarket & 5 & $6.2 \%$ \\
\hline 72 & $\begin{array}{l}\text { Distribuidora Internacional de } \\
\text { Alimentación, S.A. (Dia, S.A.) }\end{array}$ & Spain & $13,076^{* *}$ & $13,076^{* *}$ & 260 & Discount Store & 6 & ne \\
\hline 73 & Whole Foods Market, Inc. & U.S. & 12,917 & 12,917 & 551 & Supermarket & 3 & $10.2 \%$ \\
\hline 74 & Isetan Mitsukoshi Holdings Ltd. & Japan & 12,856 & 13,202 & 217 & Department Store & 9 & $-1.5 \%$ \\
\hline 75 & Co-operative Group Ltd. & U.K. & 12,652 & 16,484 & $-3,601$ & Supermarket & 1 & $8.7 \%$ \\
\hline 76 & Fast Retailing Co., Ltd. & Japan & $12,639^{* *}$ & $12,664^{* *}$ & 1,033 & Apparel/Footwear Specialty & 30 & $14.3 \%$ \\
\hline 77 & Toys "R" Us, Inc. & U.S. & 12,543 & 12,543 & $-1,036$ & Other Specialty & 38 & $-1.8 \%$ \\
\hline 78 & $\begin{array}{l}\text { China Resources Enterprise, } \\
\text { Limited }\end{array}$ & $\begin{array}{l}\text { Hong Kong } \\
\text { SAR }\end{array}$ & 12,258 & $18,877^{* *}$ & 406 & Hypermarket/Supercenter/Superstore & 5 & $22.4 \%$ \\
\hline 79 & Alliance Boots $\mathrm{GmbH}$ & Switzerland & 12,170 & $37,150^{* *}$ & 1,544 & Drug Store/Pharmacy & 14 & $1.4 \%$ \\
\hline
\end{tabular}

${ }^{1}$ Revenue and net income for the parent company or group may include results from non-retail operations ${ }_{2}$ Compound annual growth rate $\mathrm{e}=$ estimate

$\mathrm{g}=$ gross turnover as reported by company n/a = not available ne $=$ not in existence (created by merger or divestiture) * Revenue reflects wholesale sales

** Revenue includes wholesale and retail sales 


\begin{tabular}{|c|c|c|c|c|c|c|c|c|}
\hline $\begin{array}{l}\text { Retail } \\
\text { revenue } \\
\text { rank } \\
\text { (FY13) }\end{array}$ & Name of company & $\begin{array}{l}\text { Country } \\
\text { of origin }\end{array}$ & $\begin{array}{r}2013 \\
\text { net retail } \\
\text { revenue } \\
\text { (US\$m) }\end{array}$ & $\begin{array}{r}2013 \\
\text { parent } \\
\text { company/ } \\
\text { group } \\
\text { revenue }{ }^{1} \\
(\text { US\$m) }\end{array}$ & $\begin{array}{r}2013 \\
\text { parent } \\
\text { company/ } \\
\text { group } \\
\text { net income }{ }^{1} \\
(\text { US\$m) }\end{array}$ & Dominant operational format 2013 & $\begin{array}{r}\# \\
\text { countries } \\
\text { of } \\
\text { operation } \\
2013\end{array}$ & $\begin{array}{r}2008- \\
2013 \\
\text { retail } \\
\text { revenue } \\
\text { CAGR }^{2}\end{array}$ \\
\hline 80 & Nordstrom, Inc. & U.S. & 12,166 & 12,540 & 734 & Department Store & 1 & $8.0 \%$ \\
\hline 81 & Staples, Inc. & U.S. & $12,160^{\mathrm{e}}$ & 23,114 & 620 & Other Specialty & 13 & $-1.0 \%$ \\
\hline 82 & J. C. Penney Company, Inc. & U.S. & 11,859 & 11,859 & $-1,388$ & Department Store & 2 & $-8.5 \%$ \\
\hline 83 & BJ's Wholesale Club, Inc. & U.S. & $11,800^{\mathrm{e}}$ & $11,800^{\mathrm{e}}$ & $\mathrm{n} / \mathrm{a}$ & Cash \& Carry/Warehouse Club & 1 & $3.3 \%$ \\
\hline 84 & Louis Delhaize S.A. & Belgium & $11,689^{e}$ & $11,689^{e}$ & $n / a$ & Hypermarket/Supercenter/Superstore & 6 & $-4.4 \%$ \\
\hline 85 & Dixons Retail plc & U.K. & 11,560 & 11,560 & -113 & Electronics Specialty & 7 & $-2.9 \%$ \\
\hline 86 & Bed Bath and Beyond Inc. & U.S. & $11,504^{* *}$ & $11,504^{* *}$ & 1,022 & Other Specialty & 4 & $9.8 \%$ \\
\hline 87 & $\begin{array}{l}\text { Spar Holding AG (formerly SPAR } \\
\text { Österreichische Warenhandels-AG) }\end{array}$ & Austria & $11,411^{* *}$ & $11,518^{* *}$ & 251 & Supermarket & 8 & $2.2 \%$ \\
\hline 88 & S.A.C.I. Falabella & Chile & 11,377 & 12,523 & 1,004 & Department Store & 5 & $11.4 \%$ \\
\hline 89 & Metro Inc. & Canada & $11,236^{* *}$ & $11,236^{* *}$ & 711 & Supermarket & 1 & $1.2 \%$ \\
\hline 90 & E-MART Co., Ltd. & S. Korea & 11,164 & 11,992 & 438 & Hypermarket/Supercenter/Superstore & 2 & ne \\
\hline 91 & NorgesGruppen ASA & Norway & $11,054^{* *}$ & $11,483^{* *}$ & 306 & Discount Store & 1 & $6.6 \%$ \\
\hline 92 & $\begin{array}{l}\text { JD.com, Inc. (formerly Beijing } \\
\text { Jingdong Century Trade Co., Ltd. } \\
\text { and } 360 \text { buy Jingdong Inc.) }\end{array}$ & China & 10,827 & 11,202 & -8 & Non-Store & 78 & $123.6 \%$ \\
\hline 93 & L Brands, Inc. & U.S. & $10,773^{* *}$ & $10,773^{* *}$ & 903 & Apparel/Footwear Specialty & 58 & $3.6 \%$ \\
\hline 94 & Shoppers Drug Mart Corporation & Canada & 10,742 & 10,742 & 584 & Drug Store/Pharmacy & 1 & $3.3 \%$ \\
\hline 95 & J. Front Retailing Co., Ltd. & Japan & 10,411 & 11,521 & 348 & Department Store & 2 & $1.5 \%$ \\
\hline 96 & Family Dollar Stores, Inc. & U.S. & 10,391 & 10,391 & 444 & Discount Store & 1 & $8.3 \%$ \\
\hline 97 & Canadian Tire Corporation, Limited & Canada & $10,385^{\star *}$ & $11,449^{* *}$ & 548 & Other Specialty & 1 & $4.9 \%$ \\
\hline 98 & $\begin{array}{l}\text { Dairy Farm International Holdings } \\
\text { Limited }\end{array}$ & $\begin{array}{l}\text { Hong Kong } \\
\text { SAR }\end{array}$ & 10,357 & 10,357 & 513 & Supermarket & 12 & $9.0 \%$ \\
\hline 99 & Liberty Interactive Corporation & U.S. & 10,307 & 11,252 & 580 & Non-Store & 9 & $5.0 \%$ \\
\hline 100 & Ross Stores, Inc. & U.S. & 10,230 & 10,230 & 837 & Apparel/Footwear Specialty & 1 & $9.5 \%$ \\
\hline 101 & Uny Group Holdings Co., Ltd. & Japan & $10,156^{\star \star}$ & $10,373^{\star *}$ & 66 & Convenience/Forecourt Store & 3 & $-2.8 \%$ \\
\hline 102 & $\begin{array}{l}\text { Southeastern Grocers, LLC } \\
\text { (formerly BI-LO Holding, LLC) }\end{array}$ & U.S. & $10,126^{e}$ & $10,126^{e}$ & $\mathrm{n} / \mathrm{a}$ & Supermarket & 1 & $32.2 \%$ \\
\hline 103 & $\begin{array}{l}\text { Tengelmann } \\
\text { Warenhandelsgesellschaft KG }\end{array}$ & Germany & $10,072^{\mathrm{e}^{\star \star}}$ & $10,390^{* *}$ & $\mathrm{n} / \mathrm{a}$ & Home Improvement & 13 & $-12.0 \%$ \\
\hline 104 & $\begin{array}{l}\text { Chow Tai Fook Jewellery Group } \\
\text { Limited }\end{array}$ & $\begin{array}{l}\text { Hong Kong } \\
\text { SAR }\end{array}$ & $9,979^{* *}$ & $9,979^{* *}$ & 960 & Other Specialty & 6 & $33.3 \%$ \\
\hline 105 & Dansk Supermarked A/S & Denmark & 9,921 & 9,974 & 315 & Discount Store & 4 & $-0.5 \%$ \\
\hline 106 & Giant Eagle, Inc. & U.S. & $9,900^{\mathrm{ex}^{* *}}$ & $9,900^{\mathrm{e}^{* *}}$ & $\mathrm{n} / \mathrm{a}$ & Supermarket & 1 & $3.8 \%$ \\
\hline 107 & Shoprite Holdings Ltd. & S. Africa & $9,869^{\star \star}$ & $9,869^{\star *}$ & 361 & Supermarket & 15 & $11.5 \%$ \\
\hline 108 & Oxylane Groupe & France & 9,829 & 9,829 & $\mathrm{n} / \mathrm{a}$ & Other Specialty & 22 & $8.3 \%$ \\
\hline 109 & C\&A Europe & $\begin{array}{l}\text { Belgium/ } \\
\text { Germany }\end{array}$ & $9,733^{\mathrm{e}}$ & $9,733^{e}$ & $\mathrm{n} / \mathrm{a}$ & Apparel/Footwear Specialty & 21 & $3.0 \%$ \\
\hline 110 & Menard, Inc. & U.S. & $9,500^{e}$ & $9,500^{e}$ & $\mathrm{n} / \mathrm{a}$ & Home Improvement & 1 & $3.2 \%$ \\
\hline 111 & Jumbo Groep Holding B.V. & Netherlands & $9,420^{9}$ & $9,420^{9}$ & $\mathrm{n} / \mathrm{a}$ & Supermarket & 1 & $36.9 \%$ \\
\hline 112 & Globus Holding GmbH \& Co. KG & Germany & $9,349^{9}$ & $9,349^{9}$ & $n / a$ & Hypermarket/Supercenter/Superstore & 4 & $4.0 \%$ \\
\hline 113 & CP ALL Public Company Limited & Thailand & 9,286 & $9,300^{* *}$ & 347 & Convenience/Forecourt Store & 1 & $17.5 \%$ \\
\hline 114 & AutoZone, Inc. & U.S. & 9,148 & 9,148 & 1,016 & Other Specialty & 4 & $7.0 \%$ \\
\hline 115 & GameStop Corp. & U.S. & 9,040 & 9,040 & 354 & Other Specialty & 16 & $0.5 \%$ \\
\hline 116 & dm-drogerie markt GmbH + Co. KG & Germany & $9,009^{e}$ & $10,090^{9}$ & $\mathrm{n} / \mathrm{a}$ & Drug Store/Pharmacy & 12 & $10.1 \%$ \\
\hline 117 & Home Retail Group plc & U.K. & 8,929 & 8,929 & 85 & Other Specialty & 3 & $-0.8 \%$ \\
\hline 118 & SuperValu Inc. & U.S. & $8,879^{* *}$ & $17,155^{* *}$ & 182 & Supermarket & 1 & $-23.8 \%$ \\
\hline 119 & Bic Camera Inc. & Japan & 8,827 & 8,924 & 40 & Electronics Specialty & 1 & $5.8 \%$ \\
\hline 120 & Dirk Rossmann GmbH & Germany & 8,820 & 8,820 & $n / a$ & Drug Store/Pharmacy & 6 & $11.5 \%$ \\
\hline 121 & "Kesko Corporation " & Finland & $8,776^{\mathrm{e}^{* *}}$ & $12,373^{* *}$ & 245 & Supermarket & 8 & $1.5 \%$ \\
\hline
\end{tabular}

${ }^{1}$ Revenue and net income for the parent company or group may include results from non-retail operations ${ }^{2}$ Compound annual growth rate $\mathrm{n} / \mathrm{a}=$ not available ne $=$ not in existence (created by merger or divestiture) * Revenue reflects wholesale sales

** Revenue includes wholesale and retail sales 


\begin{tabular}{|c|c|c|c|c|c|c|c|c|}
\hline $\begin{array}{l}\text { Retail } \\
\text { revenue } \\
\text { rank } \\
\text { (FY13) }\end{array}$ & Name of company & $\begin{array}{l}\text { Country } \\
\text { of origin }\end{array}$ & $\begin{array}{r}2013 \\
\text { net retail } \\
\text { revenue } \\
(\text { US\$m) }\end{array}$ & $\begin{array}{r}2013 \\
\text { parent } \\
\text { company/ } \\
\text { group } \\
\text { revenue } \\
(\text { US } \$ \mathrm{~m})\end{array}$ & $\begin{array}{r}2013 \\
\text { parent } \\
\text { company/ } \\
\text { group } \\
\text { net income }{ }^{1} \\
(\text { US\$m) }\end{array}$ & Dominant operational format 2013 & $\begin{array}{r}\# \\
\text { countries } \\
\text { of } \\
\text { operation } \\
2013\end{array}$ & $\begin{array}{r}2008- \\
2013 \\
\text { retail } \\
\text { revenue } \\
\text { CAGR }^{2}\end{array}$ \\
\hline 122 & Colruyt Group & Belgium & 8,760 & $11,598^{* *}$ & 469 & Supermarket & 3 & $6.2 \%$ \\
\hline 123 & Reitan Group & Norway & 8,512 & 9,754 & 341 & Discount Store & 7 & $11.2 \%$ \\
\hline 124 & Esselunga S.p.A. & Italy & $8,474^{e}$ & $9,241^{9}$ & 279 & Hypermarket/Supercenter/Superstore & 1 & $3.8 \%$ \\
\hline 125 & Takashimaya Co., Ltd. & Japan & 8,390 & 9,087 & 196 & Department Store & 4 & $-1.8 \%$ \\
\hline 126 & $\begin{array}{l}\text { Army \& Air Force Exchange } \\
\text { Service (AAFES) }\end{array}$ & U.S. & 8,308 & 8,308 & 167 & Hypermarket/Supercenter/Superstore & 26 & $-1.3 \%$ \\
\hline 127 & $\begin{array}{l}\text { Organización Soriana, } \\
\text { S.A.B. de C.V. }\end{array}$ & Mexico & $8,240^{\star *}$ & $8,240^{* *}$ & 245 & Hypermarket/Supercenter/Superstore & 1 & $1.9 \%$ \\
\hline 128 & Steinhoff International Holdings Ltd. & S. Africa & 8,217 & 11,333 & 952 & Other Specialty & 26 & $31.5 \%$ \\
\hline 129 & $\begin{array}{l}\text { Shanghai Friendship Group } \\
\text { Incorporated Co. }\end{array}$ & China & $8,166^{* *}$ & $8,389^{* *}$ & 202 & Supermarket & 1 & $14.8 \%$ \\
\hline 130 & Hy-Vee, Inc. & U.S. & 8,014 & 8,014 & $\mathrm{n} / \mathrm{a}$ & Supermarket & 1 & $5.3 \%$ \\
\hline 131 & Beisia Group Co., Ltd. & Japan & $7,885^{e}$ & $8,524^{e}$ & $\mathrm{n} / \mathrm{a}$ & Home Improvement & 1 & $1.7 \%$ \\
\hline 132 & $\begin{array}{l}\text { Compagnie Financière } \\
\text { Richemont SA }\end{array}$ & Switzerland & 7,841 & $14,275^{* *}$ & 2,771 & Other Specialty & 170 & $20.5 \%$ \\
\hline 133 & Dollar Tree, Inc. & U.S. & 7,840 & 7,840 & 597 & Discount Store & 2 & $11.0 \%$ \\
\hline 134 & FEMSA Comercio, S.A. de C.V. & Mexico & 7,655 & 7,655 & $\mathrm{n} / \mathrm{a}$ & Convenience/Forecourt Store & 2 & $15.7 \%$ \\
\hline 135 & SHV Holdings N.V./Makro & Netherlands & 7,621 & 23,377 & 4,727 & Cash \& Carry/Warehouse Club & 6 & $6.5 \%$ \\
\hline 136 & Grupo Eroski & Spain & $7,585^{e}$ & 7,829 & -136 & Supermarket & 2 & $-6.2 \%$ \\
\hline 137 & Edion Corporation & Japan & $7,277^{\mathrm{e}^{\star *}}$ & $7,659^{* *}$ & 52 & Electronics Specialty & 1 & $-0.9 \%$ \\
\hline 138 & K's Holdings Corporation & Japan & $7,005^{* *}$ & $7,005^{* *}$ & 175 & Electronics Specialty & 1 & $4.1 \%$ \\
\hline 139 & Wegmans Food Markets, Inc. & U.S. & $7,000^{\mathrm{e}}$ & $7,000^{e}$ & $\mathrm{n} / \mathrm{a}$ & Supermarket & 1 & $7.8 \%$ \\
\hline 140 & PetSmart, Inc. & U.S. & 6,917 & 6,917 & 420 & Other Specialty & 3 & $6.4 \%$ \\
\hline 141 & Yodobashi Camera Co., Ltd. & Japan & $6,901^{e}$ & $6,901^{e}$ & $\mathrm{n} / \mathrm{a}$ & Electronics Specialty & 1 & $-0.3 \%$ \\
\hline 142 & Coop Danmark A/S & Denmark & $6,859^{* *}$ & $7,071^{* *}$ & 10 & Supermarket & 2 & $1.3 \%$ \\
\hline 143 & Kering S.A. & France & 6,732 & $12,948^{* *}$ & 53 & Apparel/Footwear Specialty & 90 & $-18.5 \%$ \\
\hline 144 & $\begin{array}{l}\text { Associated British Foods plc/ } \\
\text { Primark }\end{array}$ & U.K. & 6,673 & 20,792 & 990 & Apparel/Footwear Specialty & 8 & $17.2 \%$ \\
\hline 145 & O'Reilly Automotive, Inc. & U.S. & $6,649^{\star \star}$ & $6,649^{* *}$ & 670 & Other Specialty & 1 & $13.2 \%$ \\
\hline 146 & Dillard's, Inc. & U.S. & $6,599^{e}$ & 6,692 & 324 & Department Store & 1 & $-0.9 \%$ \\
\hline 147 & Foot Locker, Inc. & U.S. & 6,505 & 6,505 & 429 & Apparel/Footwear Specialty & 30 & $4.4 \%$ \\
\hline 148 & Pick n Pay Stores Limited & S. Africa & 6,351 & 6,351 & 59 & Supermarket & 7 & $4.8 \%$ \\
\hline 149 & Coppel S.A. de C.V. & Mexico & 6,304 & 6,304 & 850 & Department Store & 3 & $17.1 \%$ \\
\hline 150 & Lojas Americanas S.A. & Brazil & 6,247 & 6,247 & 188 & Discount Department Store & 1 & $14.0 \%$ \\
\hline 151 & BìM Birleşik Mağazalar A.Ş. & Turkey & 6,242 & 6,242 & 218 & Discount Store & 3 & $22.8 \%$ \\
\hline 152 & Dick's Sporting Goods, Inc. & U.S. & 6,213 & 6,213 & 338 & Other Specialty & 1 & $8.5 \%$ \\
\hline 153 & Central Retail Corporation Ltd. & Thailand & $6,207^{e}$ & $6,207^{e}$ & $\mathrm{n} / \mathrm{a}$ & Department Store & 4 & $17.2 \%$ \\
\hline 154 & Advance Auto Parts, Inc. & U.S. & $6,171^{* *}$ & $6,494^{* *}$ & 392 & Other Specialty & 2 & $4.4 \%$ \\
\hline 155 & Sonae, SGPS, SA & Portugal & 6,144 & 6,404 & 616 & Supermarket & 16 & $1.9 \%$ \\
\hline 156 & President Chain Store Corp. & Taiwan & $6,136^{e}$ & $6,771^{* *}$ & 312 & Convenience/Forecourt Store & 4 & $8.1 \%$ \\
\hline 157 & Bauhaus GmbH \& Co. KG & Germany & $6,073^{e}$ & $6,073^{e}$ & $\mathrm{n} / \mathrm{a}$ & Home Improvement & 17 & $8.8 \%$ \\
\hline 158 & Office Depot, Inc. & U.S. & $6,015^{e}$ & 11,242 & -20 & Other Specialty & 21 & $-4.7 \%$ \\
\hline 159 & $\begin{array}{l}\text { The Sherwin-Williams Company/ } \\
\text { Paint Stores Group }\end{array}$ & U.S. & 6,002 & $10,186^{* *}$ & 753 & Home Improvement & 8 & $4.4 \%$ \\
\hline 160 & $\begin{array}{l}\text { Defense Commissary Agency } \\
\text { (DeCA) }\end{array}$ & U.S. & 5,900 & 5,900 & $n / a$ & Supermarket & 13 & $0.3 \%$ \\
\hline 161 & $\begin{array}{l}\text { The Great Atlantic \& Pacific Tea } \\
\text { Company, Inc. }\end{array}$ & U.S. & $5,900^{\mathrm{e}}$ & $5,900^{e}$ & $\mathrm{n} / \mathrm{a}$ & Supermarket & 1 & $-9.1 \%$ \\
\hline 162 & $\begin{array}{l}\text { Belle International Holdings } \\
\text { Limited }\end{array}$ & $\begin{array}{l}\text { Hong Kong } \\
\text { SAR }\end{array}$ & 5,856 & 5,856 & 726 & Apparel/Footwear Specialty & 3 & $15.2 \%$ \\
\hline
\end{tabular}

${ }^{1}$ Revenue and net income for the parent company or group may include results from non-retail operations 2 Compound annual growth rate $\mathrm{n} / \mathrm{a}=$ not available ne $=$ not in existence (created by merger or divestiture) * Revenue reflects wholesale sales

** Revenue includes wholesale and retail sales 


\begin{tabular}{|c|c|c|c|c|c|c|c|c|}
\hline $\begin{array}{l}\text { Retail } \\
\text { revenue } \\
\text { rank } \\
\text { (FY13) }\end{array}$ & Name of company & $\begin{array}{l}\text { Country } \\
\text { of origin }\end{array}$ & $\begin{array}{r}2013 \\
\text { net retail } \\
\text { revenue } \\
(\text { US\$m) }\end{array}$ & $\begin{array}{r}2013 \\
\text { parent } \\
\text { company/ } \\
\text { group } \\
\text { revenue } \\
\text { (US\$m) }\end{array}$ & $\begin{array}{r}2013 \\
\text { parent } \\
\text { company/ } \\
\text { group } \\
\text { net income }{ }^{1} \\
(\text { US\$m) }\end{array}$ & Dominant operational format 2013 & $\begin{array}{r}\# \\
\text { countries } \\
\text { of } \\
\text { operation } \\
2013\end{array}$ & $\begin{array}{r}2008- \\
2013 \\
\text { retail } \\
\text { revenue } \\
\text { CAGR }^{2}\end{array}$ \\
\hline 163 & Next plc & U.K. & 5,843 & $5,868^{* *}$ & 868 & Apparel/Footwear Specialty & 74 & $3.8 \%$ \\
\hline 164 & Don Quijote Co., Ltd. & Japan & 5,842 & 6,063 & 238 & Discount Department Store & 2 & $5.0 \%$ \\
\hline 165 & WinCo Foods LLC & U.S. & $5,700 \mathrm{e}$ & $5,700 \mathrm{e}$ & $\mathrm{n} / \mathrm{a}$ & Supermarket & 1 & $7.3 \%$ \\
\hline 166 & OJSC Dixy Group & Russia & 5,628 & 5,679 & 96 & Supermarket & 1 & $30.2 \%$ \\
\hline 167 & Izumi Co., Ltd. & Japan & 5,564 & 5,596 & 176 & Hypermarket/Supercenter/Superstore & 1 & $3.0 \%$ \\
\hline 168 & KF Gruppen & Sweden & $5,449^{\star \star *}$ & $5,854^{* *}$ & -8 & Supermarket & 1 & $1.3 \%$ \\
\hline 169 & Deichmann SE & Germany & 5,313 & $6,110^{9}$ & $\mathrm{n} / \mathrm{a}$ & Apparel/Footwear Specialty & 23 & $8.3 \%$ \\
\hline 170 & Nike, Inc. & U.S. & 5,304 & $27,799^{* *}$ & 2,693 & Apparel/Footwear Specialty & 49 & $19.5 \%$ \\
\hline 171 & Big Lots, Inc. & U.S. & 5,302 & 5,302 & 125 & Discount Store & 2 & $2.7 \%$ \\
\hline 172 & H2O Retailing Corporation & Japan & 5,270 & 5,763 & 3 & Department Store & 1 & $2.0 \%$ \\
\hline 173 & Life Corporation & Japan & 5,226 & 5,376 & 38 & Supermarket & 1 & $2.9 \%$ \\
\hline 174 & The SPAR Group Limited & S. Africa & $5,175^{* *}$ & $5,175^{* *}$ & 129 & Supermarket & 7 & $12.1 \%$ \\
\hline 175 & Tractor Supply Company & U.S. & 5,165 & 5,165 & 328 & Other Specialty & 1 & $11.4 \%$ \\
\hline 176 & $\begin{array}{l}\text { Grupo Comercial Chedraui, } \\
\text { S.A.B. de C.V. }\end{array}$ & Mexico & 5,157 & 5,207 & 134 & Hypermarket/Supercenter/Superstore & 2 & $10.4 \%$ \\
\hline 177 & $\begin{array}{l}\text { El Puerto de Liverpool, } \\
\text { S.A.B. de C.V. }\end{array}$ & Mexico & 5,156 & 5,814 & 604 & Department Store & 1 & $9.7 \%$ \\
\hline 178 & Coop Norge, the Group & Norway & $5,133^{* *}$ & $5,362^{* *}$ & -7 & Supermarket & 1 & $3.2 \%$ \\
\hline 179 & Dashang Co., Ltd. & China & 5,047 & 5,452 & 203 & Department Store & 1 & $12.2 \%$ \\
\hline 180 & Shimamura Co., Ltd. & Japan & 5,044 & 5,044 & 267 & Apparel/Footwear Specialty & 3 & $4.1 \%$ \\
\hline 181 & Hudson's Bay Company & Canada & 5,034 & 5,034 & -249 & Department Store & 2 & $\mathrm{n} / \mathrm{a}$ \\
\hline 182 & Foodstuffs North Island Ltd. & $\begin{array}{l}\text { New } \\
\text { Zealand }\end{array}$ & $5,021^{* * *}$ & $5,021^{\mathrm{e}^{* *}}$ & 127 & Hypermarket/Supercenter/Superstore & 1 & ne \\
\hline 183 & $\begin{array}{l}\text { Emke Group/Lulu Group } \\
\text { International }\end{array}$ & UAE & $5,000^{e}$ & $5,000^{e}$ & $n / a$ & Hypermarket/Supercenter/Superstore & 10 & $18.7 \%$ \\
\hline 184 & Groupe FNAC S.A. & France & $4,932^{* *}$ & $5,187^{* *}$ & 20 & Other Specialty & 7 & ne \\
\hline 185 & MatsumotoKiyoshi Holdings Co., Ltd. & Japan & $4,919^{* *}$ & $4,949^{* *}$ & 134 & Drug Store/Pharmacy & 1 & $4.8 \%$ \\
\hline 186 & E.Land World Ltd. & S. Korea & $4,914^{\mathrm{e}^{* *}}$ & $5,683^{* *}$ & 111 & Apparel/Footwear Specialty & 15 & $11.4 \%$ \\
\hline 187 & Darty plc & U.K. & 4,821 & 4,821 & -9 & Electronics Specialty & 5 & $-9.5 \%$ \\
\hline 188 & $\begin{array}{l}\text { Chongqing Department Store } \\
\text { Co., Ltd. }\end{array}$ & China & 4,733 & 4,886 & 126 & Department Store & 1 & $36.0 \%$ \\
\hline 189 & Yonghui Superstores Co., Ltd. & China & 4,733 & 4,934 & 117 & Hypermarket/Supercenter/Superstore & 1 & $38.8 \%$ \\
\hline 190 & Ascena Retail Group, Inc. & U.S. & 4,715 & 4,715 & 151 & Apparel/Footwear Specialty & 3 & $26.7 \%$ \\
\hline 191 & Harris Teeter Supermarkets, Inc. & U.S. & 4,710 & 4,710 & 108 & Supermarket & 1 & $5.1 \%$ \\
\hline 192 & OJSC "Company M.video" & Russia & 4,657 & 4,657 & 180 & Electronics Specialty & 1 & $16.8 \%$ \\
\hline 193 & Neiman Marcus Group LTD Inc. & U.S. & 4,648 & 4,648 & 164 & Department Store & 2 & $0.2 \%$ \\
\hline 194 & Michaels Stores, Inc. & U.S. & 4,570 & 4,570 & 264 & Other Specialty & 2 & $3.7 \%$ \\
\hline 195 & Arcs Co., Ltd. & Japan & 4,554 & 4,567 & 64 & Supermarket & 1 & $13.8 \%$ \\
\hline 196 & Lenta Group & Russia & 4,539 & 4,539 & 223 & Hypermarket/Supercenter/Superstore & 1 & $23.2 \%$ \\
\hline 197 & $\begin{array}{l}\text { Nonggongshang Supermarket } \\
\text { (Group) Co. Ltd. }\end{array}$ & China & $4,538^{\mathrm{e}}$ & $4,538^{g}$ & $\mathrm{n} / \mathrm{a}$ & Supermarket & 1 & $5.2 \%$ \\
\hline 198 & Douglas Holding AG & Germany & $4,532^{\mathrm{e}}$ & $4,532^{\mathrm{e}}$ & $\mathrm{n} / \mathrm{a}$ & Other Specialty & 18 & $1.9 \%$ \\
\hline 199 & Celesio AG & Germany & 4,532 & $28,435^{* *}$ & 221 & Drug Store/Pharmacy & 7 & $-0.8 \%$ \\
\hline 200 & Sundrug Co., Ltd. & Japan & $4,474^{\star \star}$ & $4,474^{* *}$ & 157 & Drug Store/Pharmacy & 1 & $14.2 \%$ \\
\hline 201 & $\begin{array}{l}\text { Burlington Coat Factory } \\
\text { Investments Holdings, Inc. }\end{array}$ & U.S. & 4,462 & 4,462 & 44 & Department Store & 2 & $4.6 \%$ \\
\hline 202 & Tokyu Corporation & Japan & 4,432 & 10,820 & 574 & Department Store & 3 & $-5.2 \%$ \\
\hline 203 & Save Mart Supermarkets & U.S. & $4,390^{\mathrm{e}}$ & $4,390^{\mathrm{e}}$ & $\mathrm{n} / \mathrm{a}$ & Supermarket & 1 & $-3.0 \%$ \\
\hline 204 & Williams-Sonoma, Inc. & U.S. & 4,388 & 4,388 & 279 & Other Specialty & 10 & $5.5 \%$ \\
\hline 205 & DCM Holdings Co., Ltd. & Japan & 4,361 & 4,364 & 103 & Home Improvement & 1 & $0.7 \%$ \\
\hline
\end{tabular}

${ }^{1}$ Revenue and net income for the parent company or group may include results from non-retail operations

2 Compound annual growth rate $\mathrm{n} / \mathrm{a}=$ not available ne $=$ not in existence (created by merger or divestiture) * Revenue reflects wholesale sales

** Revenue includes wholesale and retail sales 


\begin{tabular}{|c|c|c|c|c|c|c|c|c|}
\hline $\begin{array}{l}\text { Retail } \\
\text { revenue } \\
\text { rank } \\
\text { (FY13) }\end{array}$ & Name of company & $\begin{array}{l}\text { Country } \\
\text { of origin }\end{array}$ & $\begin{array}{r}2013 \\
\text { net retail } \\
\text { revenue } \\
(\text { US\$m) }\end{array}$ & $\begin{array}{r}2013 \\
\text { parent } \\
\text { company/ } \\
\text { group } \\
\text { revenue }^{1} \\
\text { (US\$m) }\end{array}$ & $\begin{array}{r}2013 \\
\text { parent } \\
\text { company/ } \\
\text { group } \\
\text { net income }{ }^{1} \\
(\mathrm{US} \$ \mathrm{~m})\end{array}$ & Dominant operational format 2013 & $\begin{array}{r}\# \\
\text { countries } \\
\text { of } \\
\text { operation } \\
2013\end{array}$ & $\begin{array}{r}2008- \\
2013 \\
\text { retail } \\
\text { revenue } \\
\text { CAGR }^{2}\end{array}$ \\
\hline 206 & O’Key Group S.A. & Russia & 4,330 & 4,387 & 157 & Hypermarket/Supercenter/Superstore & 1 & $22.3 \%$ \\
\hline 207 & Valor Co., Ltd. & Japan & 4,324 & 4,537 & 92 & Supermarket & 2 & $5.8 \%$ \\
\hline 208 & Iceland Topco Limited & U.K. & $4,309^{* *}$ & $4,309^{* *}$ & $\mathrm{n} / \mathrm{a}$ & Supermarket & 7 & $5.4 \%$ \\
\hline 209 & Coach, Inc. & U.S. & $4,303^{e}$ & $4,806^{* *}$ & 781 & Other Specialty & 17 & $9.6 \%$ \\
\hline 210 & Demoulas Super Markets, Inc. & U.S. & $4,300^{\mathrm{e}}$ & $4,300^{\mathrm{e}}$ & $n / a$ & Supermarket & 1 & $9.0 \%$ \\
\hline 211 & Barnes \& Noble, Inc. & U.S. & 4,295 & $6,381^{* *}$ & -47 & Other Specialty & 1 & $-3.5 \%$ \\
\hline 212 & $\begin{array}{l}\text { Berkshire Hathaway Inc./Retail } \\
\text { operations }\end{array}$ & U.S. & 4,288 & 182,150 & 19,845 & Other Specialty & 8 & $6.7 \%$ \\
\hline 213 & Arcadia Group Limited & U.K. & $4,277^{* *}$ & $4,277^{* *}$ & 693 & Apparel/Footwear Specialty & 46 & $8.2 \%$ \\
\hline 214 & SM Investments Corporation & Philippines & 4,276 & 5,988 & 904 & Department Store & 1 & $9.5 \%$ \\
\hline 215 & Lawson, Inc. & Japan & $4,229^{* *}$ & $4,877^{* *}$ & 384 & Convenience/Forecourt Store & 4 & $6.6 \%$ \\
\hline 216 & GS Retail Co., Ltd. & S. Korea & 4,223 & 4,332 & 110 & Convenience/Forecourt Store & 1 & $7.4 \%$ \\
\hline 217 & Signet Jewelers Limited & Bermuda & 4,209 & 4,209 & 368 & Other Specialty & 3 & $4.7 \%$ \\
\hline 218 & HORNBACH-Baumarkt-AG Group & Germany & 4,202 & 4,202 & 75 & Home Improvement & 9 & $3.9 \%$ \\
\hline 219 & Abercrombie \& Fitch Co. & U.S. & 4,117 & 4,117 & 55 & Apparel/Footwear Specialty & 22 & $3.1 \%$ \\
\hline 220 & RONA Inc. & Canada & $4,072^{* *}$ & $4,072^{* *}$ & -149 & Home Improvement & 1 & $-3.0 \%$ \\
\hline 221 & Belk, Inc. & U.S. & 4,038 & 4,038 & 159 & Department Store & 1 & $2.9 \%$ \\
\hline 222 & Agrokor d.d. & Croatia & $4,011^{* *}$ & $5,291^{* *}$ & 6 & Supermarket & 3 & $\mathrm{n} / \mathrm{a}$ \\
\hline 223 & $\begin{array}{l}\text { East Japan Railway Company } \\
\text { (JR East) }\end{array}$ & Japan & 4,005 & 27,002 & 2,008 & Convenience/Forecourt Store & 1 & $-0.7 \%$ \\
\hline 224 & Forever 21, Inc. & U.S. & $4,000^{e}$ & $4,000^{e}$ & $n / a$ & Apparel/Footwear Specialty & 39 & $18.7 \%$ \\
\hline 225 & PRADA Group & Italy & 3,989 & 4,776 & 849 & Apparel/Footwear Specialty & 42 & $28.0 \%$ \\
\hline 226 & Axfood AB & Sweden & 3,989 & $5,763^{* *}$ & 153 & Supermarket & 1 & $2.4 \%$ \\
\hline 227 & Gruppo Eurospin & Italy & $3,985^{\mathrm{e}^{\star *}}$ & $4,317^{9^{* *}}$ & $\mathrm{n} / \mathrm{a}$ & Discount Store & 2 & $7.8 \%$ \\
\hline 228 & Groupe Vivarte & France & 3,977 & 3,977 & $\mathrm{n} / \mathrm{a}$ & Apparel/Footwear Specialty & 64 & $0.9 \%$ \\
\hline 229 & $\begin{array}{l}\text { Norma Lebensmittelfilialbetrieb } \\
\text { Stiftung \& Co. KG }\end{array}$ & Germany & $3,958^{\mathrm{e}}$ & $3,958^{e}$ & $\mathrm{n} / \mathrm{a}$ & Discount Store & 4 & $3.2 \%$ \\
\hline 230 & Roundy's, Inc. & U.S. & 3,950 & 3,950 & 35 & Supermarket & 1 & $0.4 \%$ \\
\hline 231 & Systembolaget $A B$ & Sweden & 3,948 & 3,948 & 44 & Other Specialty & 1 & $3.9 \%$ \\
\hline 232 & Academy Sports + Outdoors & U.S. & $3,900^{\mathrm{e}}$ & $3,900^{\mathrm{e}}$ & $n / a$ & Other Specialty & 1 & $11.5 \%$ \\
\hline 233 & Tsuruha Holdings Inc. & Japan & 3,864 & 3,865 & 148 & Drug Store/Pharmacy & 2 & $9.1 \%$ \\
\hline 234 & Heiwado Co., Ltd. & Japan & 3,863 & 4,066 & 81 & Hypermarket/Supercenter/Superstore & 2 & $-0.4 \%$ \\
\hline 235 & Joshin Denki Co., Ltd. & Japan & $3,863^{* *}$ & $4,014^{* *}$ & 30 & Electronics Specialty & 1 & $2.1 \%$ \\
\hline 236 & Stater Bros. Holdings Inc. & U.S. & 3,860 & 3,860 & 30 & Supermarket & 1 & $1.2 \%$ \\
\hline 237 & Müller Ltd. \& Co. KG & Germany & $3,854^{\mathrm{e}}$ & $4,582^{\mathrm{ge}}$ & $\mathrm{n} / \mathrm{a}$ & Drug Store/Pharmacy & 7 & $6.5 \%$ \\
\hline 238 & XXXLutz Group & Austria & $3,852^{\mathrm{e}}$ & $3,852^{\mathrm{e}}$ & $\mathrm{n} / \mathrm{a}$ & Other Specialty & 8 & $4.7 \%$ \\
\hline 239 & Woolworths Holdings Limited & S. Africa & 3,834 & 3,834 & 289 & Department Store & 17 & $13.4 \%$ \\
\hline 240 & Nitori Holdings Co., Ltd. & Japan & 3,831 & 3,895 & 386 & Other Specialty & 3 & $9.5 \%$ \\
\hline 241 & SMU S.A. & Chile & $3,823^{* *}$ & $3,857^{* *}$ & $-1,073$ & Supermarket & 2 & $29.8 \%$ \\
\hline 242 & Lagardère Services SA & France & 3,810 & 4,974 & $n / a$ & Other Specialty & 34 & $4.3 \%$ \\
\hline 243 & $\begin{array}{l}\text { Reinalt-Thomas Corporation } \\
\text { (dba Discount Tire/America's Tire) }\end{array}$ & U.S. & $3,800^{\mathrm{e}}$ & $3,800^{\mathrm{e}}$ & $n / a$ & Other Specialty & 1 & $9.5 \%$ \\
\hline 244 & Tiffany \& Co. & U.S. & $3,800^{e}$ & $4,031^{* *}$ & 181 & Other Specialty & 25 & $7.1 \%$ \\
\hline 245 & Ralph Lauren Corporation & U.S. & 3,798 & $7,450^{* *}$ & 776 & Apparel/Footwear Specialty & 47 & $14.4 \%$ \\
\hline 246 & Liquor Control Board of Ontario & Canada & $3,773^{e}$ & $4,750^{* *}$ & 1,659 & Other Specialty & 1 & $3.1 \%$ \\
\hline 247 & Magazine Luiza SA & Brazil & 3,758 & 4,063 & 53 & Electronics Specialty & 1 & $27.5 \%$ \\
\hline 248 & HTM-Group & France & $3,706^{e}$ & $3,706^{e}$ & $\mathrm{n} / \mathrm{a}$ & Electronics Specialty & 1 & $19.1 \%$ \\
\hline 249 & Welcia Holdings Co., Ltd. & Japan & 3,705 & 3,705 & 89 & Drug Store/Pharmacy & 2 & ne \\
\hline 250 & Overwaitea Food Group & Canada & $3,700^{\mathrm{e}}$ & $3,700^{\mathrm{e}}$ & $\mathrm{n} / \mathrm{a}$ & Supermarket & 1 & $6.5 \%$ \\
\hline
\end{tabular}

${ }^{1}$ Revenue and net income for the parent company or group may include results from non-retail operations 2 Compound annual growth rate $\mathrm{n} / \mathrm{a}=$ not available ne $=$ not in existence (created by merger or divestiture) * Revenue reflects wholesale sales

** Revenue includes wholesale and retail sales 


\begin{tabular}{|c|c|}
\hline A.S. Watson Group & 51 \\
\hline Abercrombie \& Fitch Co. & 219 \\
\hline Academy Sports + Outdoors & 232 \\
\hline Advance Auto Parts, Inc. & 154 \\
\hline Aeon Co., Ltd. & 17 \\
\hline Agrokor d.d. & 222 \\
\hline Albertson's LLC & 43 \\
\hline Aldi Einkauf GmbH \& Co. oHG & 8 \\
\hline Alliance Boots GmbH & 79 \\
\hline Amazon.com, Inc. & 15 \\
\hline Apple Inc. / Apple Retail Stores & 46 \\
\hline Arcadia Group Limited & 213 \\
\hline Arcs Co., Ltd. & 195 \\
\hline Army \& Air Force Exchange Service (AAFES) & 126 \\
\hline Ascena Retail Group, Inc. & 190 \\
\hline Associated British Foods plc / Primark & 144 \\
\hline AutoZone, Inc. & 114 \\
\hline Axfood $A B$ & 226 \\
\hline Barnes \& Noble, Inc. & 211 \\
\hline Bauhaus GmbH \& Co. KG & 157 \\
\hline Bed Bath and Beyond Inc. & 86 \\
\hline Beisia Group Co., Ltd. & 131 \\
\hline Belk, Inc. & 221 \\
\hline Belle International Holdings Limited & 162 \\
\hline Berkshire Hathaway Inc. / Retail operations & 212 \\
\hline Best Buy Co., Inc. & 25 \\
\hline Bic Camera Inc. & 119 \\
\hline Big Lots, Inc. & 171 \\
\hline Bì Birleşik Mağazalar A.Ş. & 151 \\
\hline BJ's Wholesale Club, Inc. & 83 \\
\hline Burlington Coat Factory Investments Holdings, Inc. & 201 \\
\hline C\&A Europe & 109 \\
\hline Canadian Tire Corporation, Limited & 97 \\
\hline Carrefour S.A. & 3 \\
\hline Casino Guichard-Perrachon S.A. & 13 \\
\hline Celesio AG & 199 \\
\hline Cencosud S.A. & 47 \\
\hline Central Retail Corporation Ltd. & 153 \\
\hline Centres Distributeurs E. Leclerc & 23 \\
\hline China Resources Enterprise, Limited & 78 \\
\hline Chongqing Department Store Co., Ltd. & 188 \\
\hline Chow Tai Fook Jewellery Group Limited & 104 \\
\hline Coach, Inc. & 209 \\
\hline Colruyt Group & 122 \\
\hline Compagnie Financière Richemont SA & 132 \\
\hline Conad Consorzio Nazionale, Dettaglianti Soc. Coop. a.r.I. & 67 \\
\hline Coop Danmark A/S & 142 \\
\hline Coop Group & 50 \\
\hline Coop Italia & 63 \\
\hline Coop Norge, the Group & 178 \\
\hline Co-operative Group Ltd. & 75 \\
\hline Coppel S.A. de C.V. & 149 \\
\hline Costco Wholesale Corporation & 2 \\
\hline CP ALL Public Company Limited & 113 \\
\hline CVS Caremark Corporation & 12 \\
\hline Dairy Farm International Holdings Limited & 98 \\
\hline Dansk Supermarked A/S & 105 \\
\hline Darty plc & 187 \\
\hline Dashang Co., Ltd. & 179 \\
\hline DCM Holdings Co., Ltd. & 205 \\
\hline Defense Commissary Agency (DeCA) & 160 \\
\hline Deichmann SE & 169 \\
\hline Delhaize Group SA & 33 \\
\hline Demoulas Super Markets, Inc. & 210 \\
\hline
\end{tabular}

Dick's Sporting Goods, Inc. 152

Dillard's, Inc. 146

\begin{tabular}{ll}
\hline Dirk Rossmann GmbH & 120
\end{tabular}

Distribuidora Internacional de Alimentación, S.A. (Dia, S.A.) 72

\begin{tabular}{lr}
\hline Dixons Retail plc & 85
\end{tabular}

dm-drogerie markt GmbH + Co. KG 116

Dollar General Corporation 56

Dollar Tree, Inc. 133

\begin{tabular}{ll}
\hline Don Quijote Co., Ltd. & 164
\end{tabular}

\begin{tabular}{ll}
\hline Douglas Holding AG & 198 \\
\hline
\end{tabular}

$\begin{array}{ll}\text { E.Land World Ltd. } & 186\end{array}$

East Japan Railway Company (JR East) 223

\begin{tabular}{lr}
\hline Edeka Zentrale AG \& Co. KG & 16
\end{tabular}

\begin{tabular}{ll}
\hline Edion Corporation & 137
\end{tabular}

El Corte Inglés, S.A. $\quad 66$

\begin{tabular}{lr}
\hline El Puerto de Liverpool, S.A.B. de C.V. & 177
\end{tabular}

\begin{tabular}{lr}
\hline E-MART Co., Ltd. & 90
\end{tabular}

Emke Group / Lulu Group International $\quad 183$

$\begin{array}{lr}\text { Empire Company Limited } & 48\end{array}$

\begin{tabular}{ll}
\hline Esselunga S.p.A. & 124
\end{tabular}

\begin{tabular}{ll}
\hline Family Dollar Stores, Inc. & 96
\end{tabular}

\begin{tabular}{ll}
\hline Fast Retailing Co., Ltd. & 76
\end{tabular}

\begin{tabular}{ll}
\hline FEMSA Comercio, S.A. de C.V. & 134
\end{tabular}

Foodstuffs North Island Ltd. 182

\begin{tabular}{ll}
\hline Foot Locker, Inc. & 147
\end{tabular}

\begin{tabular}{ll}
\hline Forever 21, Inc. & 224 \\
\hline
\end{tabular}

\begin{tabular}{ll}
\hline GameStop Corp. & 115 \\
\hline
\end{tabular}

\begin{tabular}{ll}
\hline Giant Eagle, Inc. & 106
\end{tabular}

Globus Holding GmbH \& Co. KG 112

Gome Home Appliance Group $\quad 69$

Groupe Adeo SA $\quad 54$

Groupe Auchan SA 14

Groupe FNAC S.A. 184

Groupe Vivarte 228

Grupo Comercial Chedraui, S.A.B. de C.V. 176

Grupo Eroski 136

Gruppo Eurospin $\quad 227$

GS Retail Co., Ltd. $\quad 216$

H \& M Hennes \& Mauritz AB $\quad 49$

H.E. Butt Grocery Company 45

H2O Retailing Corporation $\quad 172$

Harris Teeter Supermarkets, Inc. 191

Heiwado Co., Ltd. 234

Home Retail Group plc 117

HORNBACH-Baumarkt-AG Group 218

HTM-Group 248

Hudson's Bay Company 181

Hy-Vee, Inc. 130

ICA Gruppen AB $\quad 65$

Iceland Topco Limited 208

Inditex, S.A. $\quad 44$

Isetan Mitsukoshi Holdings Ltd. $\quad 74$

ITM Développement International (Intermarché) 27

\begin{tabular}{lr}
\hline Izumi Co., Ltd. & 167
\end{tabular}

J Sainsbury plc 26

\begin{tabular}{ll}
\hline J. C. Penney Company, Inc. & 82 \\
\hline J. Front Retaling Co., Ltd. & 95
\end{tabular}

J. Front Retailing Co., Ltd. 95

JD.com, Inc. (formerly Beijing Jingdong Century Trade Co., Ltd. 92

and 360 buy Jingdong Inc.)

Jerónimo Martins, SGPS, S.A. $\quad 62$

John Lewis Partnership plc 68

Joshin Denki Co., Ltd. $\quad 235$

Jumbo Groep Holding B.V. $\quad 111$

Kering S.A. 143

\begin{tabular}{ll}
\hline Kesko Corporation & 121
\end{tabular} 


\begin{tabular}{|c|c|}
\hline KF Gruppen & 168 \\
\hline Kingfisher plc & 57 \\
\hline Kohl's Corporation & 52 \\
\hline Koninklijke Ahold N.V. & 24 \\
\hline K's Holdings Corporation & 138 \\
\hline L Brands, Inc. & 93 \\
\hline Lagardère Services SA & 242 \\
\hline Lawson, Inc. & 215 \\
\hline Lenta Group & 196 \\
\hline Liberty Interactive Corporation & 99 \\
\hline Life Corporation & 173 \\
\hline Liquor Control Board of Ontario & 246 \\
\hline Loblaw Companies Limited & 31 \\
\hline Lojas Americanas S.A. & 150 \\
\hline Lotte Shopping Co., Ltd. & 40 \\
\hline Louis Delhaize S.A. & 84 \\
\hline Lowe's Companies, Inc. & 20 \\
\hline LVMH Moët Hennessy-Louis Vuitton S.A. & 41 \\
\hline Macy's, Inc. & 34 \\
\hline Magazine Luiza SA & 247 \\
\hline Marks and Spencer Group plc & 60 \\
\hline MatsumotoKiyoshi Holdings Co., Ltd. & 185 \\
\hline Meijer, Inc. & 64 \\
\hline Menard, Inc. & 110 \\
\hline Mercadona, S.A. & 42 \\
\hline Metro $\mathrm{Ag}^{3}$ & 7 \\
\hline Metro Inc. & 89 \\
\hline Michaels Stores, Inc. & 194 \\
\hline Migros-Genossenschafts Bund & 38 \\
\hline Müller Ltd. \& Co. KG & 237 \\
\hline Neiman Marcus Group LTD Inc. & 193 \\
\hline Next plc & 163 \\
\hline Nike, Inc. & 170 \\
\hline Nitori Holdings Co., Ltd. & 240 \\
\hline Nonggongshang Supermarket (Group) Co. Ltd. & 197 \\
\hline Nordstrom, Inc. & 80 \\
\hline NorgesGruppen ASA & 91 \\
\hline Norma Lebensmittelfilialbetrieb Stiftung \& Co. KG & 229 \\
\hline O'Key Group S.A. & 206 \\
\hline Office Depot, Inc. & 158 \\
\hline OJSC "Company M.video" & 192 \\
\hline OJSC "Magnit" & 55 \\
\hline OJSC Dixy Group & 166 \\
\hline O'Reilly Automotive, Inc. & 145 \\
\hline Organización Soriana, S.A.B. de C.V. & 127 \\
\hline Otto (GmbH \& Co KG) & 70 \\
\hline Overwaitea Food Group & 250 \\
\hline Oxylane Groupe & 108 \\
\hline PetSmart, Inc. & 140 \\
\hline Pick n Pay Stores Limited & 148 \\
\hline PRADA Group & 225 \\
\hline President Chain Store Corp. & 156 \\
\hline Publix Super Markets, Inc. & 32 \\
\hline Ralph Lauren Corporation & 245 \\
\hline Reinalt-Thomas Corporation (dba Discount Tire/America's Tire) & 243 \\
\hline Reitan Group & 123 \\
\hline Rewe Combine & 21 \\
\hline Rite Aid Corporation & 37 \\
\hline RONA Inc. & 220 \\
\hline Ross Stores, Inc. & 100 \\
\hline Roundy's, Inc. & 230 \\
\hline S Group & 71 \\
\hline S.A.C.I. Falabella & 88 \\
\hline Safeway Inc. & 30 \\
\hline Save Mart Supermarkets & 203 \\
\hline
\end{tabular}

Sears Holdings Corp.

Shanghai Friendship Group Incorporated Co. 129

Shimamura Co., Ltd. 180

Shoppers Drug Mart Corporation $\quad 94$

Shoprite Holdings Ltd. 107

SHV Holdings N.V. / Makro 135

Signet Jewelers Limited $\quad 217$

SM Investments Corporation $\quad 214$

SMU S.A. 241

Sonae, SGPS, SA 155

Southeastern Grocers, LLC (formerly BI-LO Holding, LLC) 102

Spar Holding AG (formerly SPAR Österreichische 87

Warenhandels-AG)

Staples, Inc. $\quad 81$

Stater Bros. Holdings Inc. $\quad 236$

Steinhoff International Holdings Ltd. 128

Sundrug Co., Ltd. 200

Suning Commerce Group Co., Ltd. $\quad 59$

SuperValu Inc. 118

Systembolaget $A B \quad 231$

Système U, Centrale Nationale 39

Takashimaya Co., Ltd. 125

$\begin{array}{ll}\text { Target Corporation } & 10\end{array}$

Tengelmann Warenhandelsgesellschaft KG 103

Tesco PLC 5

The Gap, Inc. 61

The Great Atlantic \& Pacific Tea Company, Inc. 161

The Home Depot, Inc. 9

The IKEA Group (INGKA Holding B.V.) 28

The Kroger Co. 6

The Sherwin-Williams Company / Paint Stores Group 159

The SPAR Group Limited $\quad 174$

The TJX Companies, Inc. $\quad 36$

Tiffany \& Co. 244

Tokyu Corporation 202

Toys "R" Us, Inc.

Tractor Supply Company 175

Tsuruha Holdings Inc. 233

Uny Group Holdings Co., Ltd. 101

Valor Co., Ltd. $\quad 207$

Walgreen Co. 11

Wal-Mart Stores, Inc. 1

Wegmans Food Markets, Inc. 139

Welcia Holdings Co., Ltd. $\quad 249$

Wesfarmers Limited 22

Whole Foods Market, Inc.

Williams-Sonoma, Inc. 204

WinCo Foods LLC 165

Wm Morrison Supermarkets PLC 35

Woolworths Holdings Limited $\quad 239$

Woolworths Limited 18

X5 Retail Group N.V. 58

XXXLutz Group 238

Yamada Denki Co., Ltd. $\quad 53$

Yodobashi Camera Co., Ltd. 141

Yonghui Superstores Co., Ltd. 189

Please visit our website www.deloitte.com/consumerbusiness for

more details 


\section{Global Powers of Retailing Top 250 highlights}

\section{3 a challenging year for retailers as global economy decelerates}

As a whole, 2013 was another challenging year for retailers. Europe remained in recession during most of 2013, although it began to recover modestly by the end of the year and heading into 2014 - but growth remained poor. The economy continued to be afflicted by weak credit markets, fiscal austerity, and weak export markets. The U.S. economy grew slowly in 2013 largely due to a tightening of fiscal policy. Wages were relatively stagnant except for households at the upper end. In Japan, there were signs of an economic recovery owing mainly to the government's economic stimulus measures, but the economy slowed in the second half of the year. Efforts to create some inflation did not yield wage gains, and real purchasing power for consumers continued to decline.

The Chinese economy decelerated sharply in 2013, and similar slow growth continued into 2014. Inflation decelerated and producer prices declined, indicating lots of excess capacity in the economy. The government cracked down on lavish spending by officials and luxury gift giving, creating problems for high-end retailing. On the positive side, a shortage of labor boosted wages.

Many emerging countries saw their economies decelerate during this period as capital flowed back to the United States following talks of a change in U.S. monetary policy. Emerging market central banks tightened monetary policy in order to stabilize their currencies. This had a chilling effect on investment and slowed growth.

The continued weak global economy left many consumers financially constrained and retail sales under pressure. Revenue growth for the Top 250 Global Powers of Retailing, which began decelerating in 2011, continued to slow in 2013. Sales-weighted, currency-adjusted retail revenue increased 4.1 percent in 2013 for the Top 250, following a 4.9 percent gain in 2012, 5.1 percent growth in 2011, and an increase of 5.3 percent in 2010. Although growth continued to decelerate, nearly 80 percent of the Top 250 (199 companies) posted an increase in retail revenue in 2013.

Despite softer growth, profitability for the Top 250 improved. Net income/loss figures were available for 195 of the Top 250 companies in 2013. More than 90 percent of the reporting companies (179) were profitable, a slight increase over 2012 results. The composite net profit margin advanced to 3.4 percent from 3.1 percent the year before. A higher profit margin boosted composite return on assets to 5.3 percent from 5.0 percent in 2012. (Note: Comparisons with prior year Top 250 results should be interpreted with caution due to changes in the composition of the Top 250 group over time).
Net profits for Top 250 companies that are not primarily retailers are excluded from the composite net profit margin and return on assets calculations. Because these companies do not derive the majority of their revenue from retail operations, their consolidated profits mostly reflect their non-retail activities. Nine such companies were excluded in 2013: Alliance Boots, Apple, Associated British Foods, Berkshire Hathaway, Celesio, East Japan Railway, Nike, SHV Holdings, and Tokyu Corp.

The Top 250 Global Powers of Retailing generated retail revenue approaching $\$ 4.4$ trillion in 2013 , with an average size of more than $\$ 17.4$ billion (all monetary amounts shown in U.S. dollars). To earn a spot on the list required fiscal 2013 retail revenue of $\$ 3.7$ billion, down from 2012's $\$ 3.8$ billion. The lower threshold is the result of a decision to exclude convenience store companies from consideration if the majority of their retail revenue is derived from the sale of motor fuel. This resulted in the elimination of seven former Top 250 companies, which made room at the bottom of the list for newcomers.

Competition to be among the Top 250 is keen at the bottom of the list as most retailers are not mega-sized companies. More than one quarter of the Top 250 (68 companies) had retail revenue of less than $\$ 5$ billion in 2013. Only 46 companies, or less than 20 percent, had retail sales of $\$ 20$ billion or greater. Knocking on the door were 39 companies between $\$ 3.0$ billion and $\$ 3.7$ billion in 2013 retail revenue.

Top 250 quick stats 2013

- \$4.35 trillion - aggregate retail revenue of Top 250

- \$17.42 billion - average size of Top 250 (retail revenue)

- $\$ 3.7$ billion - minimum retail revenue required to be among Top 250

- $4.1 \%$ - composite year-over-year retail revenue growth

- $4.2 \%$ - 2008-2013 composite compound annual growth rate in retail revenue

- $3.4 \%$ - composite net profit margin

- $5.3 \%$ - composite return on assets

- $24.2 \%$ - percent of Top 250 retail revenue from foreign operations

- 10.2 - average \# of countries with retail operations per Top 250 company

- $65.2 \%$ - Top 250 retailers with foreign operations 


\begin{tabular}{|c|c|c|c|c|c|c|c|c|c|}
\hline $\begin{array}{l}\text { Top } 250 \\
\text { rank }\end{array}$ & Name of company & $\begin{array}{l}\text { Country } \\
\text { of origin }\end{array}$ & $\begin{array}{r}2013 \\
\text { Retail } \\
\text { revenue } \\
\text { (US\$\$mil) }\end{array}$ & $\begin{array}{r}2013 \\
\text { Retail } \\
\text { revenue } \\
\text { growth }\end{array}$ & $\begin{array}{r}2013 \\
\text { Net } \\
\text { profit } \\
\text { margin }\end{array}$ & $\begin{array}{r}2013 \\
\text { Return } \\
\text { on } \\
\text { assets }\end{array}$ & $\begin{array}{r}\text { CAGR }^{*} \\
\text { Retail } \\
\text { revenue } \\
2008-2013\end{array}$ & $\begin{array}{l}\text { \# countries } \\
\text { of operation }\end{array}$ & $\begin{array}{r}\% \text { retail } \\
\text { revenue } \\
\text { from foreign } \\
\text { operations }\end{array}$ \\
\hline 2 & $\begin{array}{l}\text { Costco Wholesale } \\
\text { Corporation }\end{array}$ & U.S. & 105,156 & $6.1 \%$ & $2.0 \%$ & $6.8 \%$ & $7.7 \%$ & 9 & $28.2 \%$ \\
\hline 5 & Tesco PLC & U.K. & 98,631 & $-2.0 \%$ & $1.5 \%$ & $1.9 \%$ & $2.9 \%$ & 13 & $32.3 \%$ \\
\hline 6 & The Kroger Co. & U.S. & 98,375 & $1.7 \%$ & $1.6 \%$ & $5.2 \%$ & $5.3 \%$ & 1 & $0.0 \%$ \\
\hline 7 & Metro $\mathrm{Ag}^{1}$ & Germany & $86,393^{e}$ & $-2.5 \%$ & $0.7 \%$ & $1.4 \%$ & $-0.9 \%$ & 32 & $62.3 \%$ \\
\hline 8 & $\begin{array}{l}\text { Aldi Einkauf GmbH \& } \\
\text { Co. oHG }\end{array}$ & Germany & $81,090^{\mathrm{e}}$ & $4.7 \%$ & $n / a$ & $\mathrm{n} / \mathrm{a}$ & $5.5 \%$ & 17 & $59.2 \%$ \\
\hline Top $10^{2}$ & & & $\$ 1,294,698$ & $2.0 \%$ & $2.8 \%$ & $6.0 \%$ & $3.0 \%$ & $16.5^{3}$ & $32.5 \%$ \\
\hline Top $250^{2}$ & & & $\$ 4,354,562$ & $4.1 \%$ & $3.4 \%$ & $5.3 \%$ & $4.2 \%$ & $10.2^{3}$ & $24.2 \%$ \\
\hline
\end{tabular}

${ }^{*}$ Compound annual growth rate

${ }^{1}$ Metro changed its fiscal year from end of December to end of September. Fiscal 2013 results reported here include the 9 months ended 30 September 2013 plus the quarter ended 31 December 2013 to create a 12-month period equilvalent to prior years.

\author{
2 Sales-weighted, currency-adjusted composites \\ 3 Average \\ $\mathrm{e}=$ estimate \\ $\mathrm{n} / \mathrm{a}=$ not available
}

Sources: Published company data and Planet Retail

Revenue declines for three top 10 retailers; Costco moves into second place

Costco's steady sales growth boosted the company into second place in 2013 from third in 2012, continuing its ascent up the top 10 leader board. Carrefour, Schwarz Group, Tesco, and Kroger - ranked in marginal descending order - all achieved 2013 retail revenue of $\$ 98+$ billion. Despite lower sales in 2013, Carrefour, which ranked fourth in 2012, assumed third place, while Schwarz Group moved up two spots into fourth place. Tesco dropped from second place in 2012 to fifth in 2013 as a result of both declining sales and a weaker British pound against the U.S. dollar. Sixth-ranked Kroger may overtake all three of these companies in the future as a result of its January 2014 acquisition of Harris Teeter Supermarkets, which had revenues of $\$ 4.7$ billion in fiscal year 2013 .

Further down the league table, Metro - despite a 2.5 percent sales decline - maintained its ranking as the world's seventh largest retailer based on 2013 annualized sales. (Metro changed its fiscal year from the end of December to the end of September. The company's 2013 results reported here include the 9 months ended 30 September - its shortened fiscal year - plus the three months ended 31 December to create a 12-month period equivalent to prior years.) Aldi overtook Home Depot on the back of a stronger euro, moving into eighth place. Tenth-ranked Target, which joined the top 10 for the first time in 2012, retained its position. However, it will likely be surpassed in 2014 by eleventh-ranked Walgreen, which acquired the remaining 55 percent of Alliance Boots in August 2014.
Compared with the Top 250 overall, the 10 largest retailers have a much bigger global footprint. On average, the top 10 had retail operations in 16.5 countries compared with 10.2 countries for the Top 250. Nearly one-third of the top 10's total retail revenue came from foreign operations, while the entire group derived about one-quarter of its collective business outside the retailers' home country. Four of the world's largest retailers - Carrefour, Schwarz, Metro, and Aldi - depended on foreign markets for the majority of their sales. Target's expansion into Canada in 2013 left Kroger as the lone single-country operator among the world's 10 largest retailers.

Changes in the overall ranking from year-to-year are generally driven by increases or decreases in companies' sales. However, a stronger currency vis-à-vis the dollar in 2013 means that companies reporting in that currency may rank higher in 2013 than they did in 2012, all other things being equal. Conversely, companies reporting in a weaker currency may rank lower. Among the major currencies in 2013, the euro strengthened against the dollar, as did China's yuan renminbi to a lesser degree. The Japanese yen, South African rand, Brazilian real, and Indian rupee all depreciated sharply while the British pound was slightly weaker. 


\title{
Global Powers of Retailing geographical analysis
}

\author{
For purposes of geographical analysis, companies are assigned to a region based on their \\ headquarters location, which may not always coincide with where they derive the majority of \\ their sales. Although many companies derive sales from outside their region, 100 percent of \\ each company's sales are accounted for within that company's region.
}

Growth falls to four-year low for European and North American retailers in 2013

The European region, with 90 companies, accounted for the largest share of the world's Top 250 retailers in 2013. However, North America, with 88 companies averaging nearly $\$ 22$ billion in retail sales, maintained the largest share of Top 250 revenue. Despite their number and size, the European and North American retailers posted the lowest composite growth rates in 2013 at 2.6 percent and 3.1 percent, respectively. For both regions, top-line revenue grew at the slowest pace since 2009.

Nearly one-quarter of the Top 250's European retailers (22 of 90 companies) and North American retailers (20 of 88 companies) experienced declining retail revenue in 2013. Many of these companies continue to rationalize their portfolios and disengage from difficult markets with the effect of reducing the overall size of their businesses.

\section{Region/country profiles, 2013}

\begin{tabular}{lrrrr} 
& $\begin{array}{r}\text { \# } \\
\text { retail revenue } \\
\text { (US\$\$়mil) }\end{array}$ & $\begin{array}{r}\text { Share of } \\
\text { Top 250 } \\
\text { Companies }\end{array}$ & $\begin{array}{r}\text { Share of } \\
\text { Top 250 } \\
\text { Revenue }\end{array}$ \\
\hline Top 250 & 250 & $\$ 17,418$ & $100.0 \%$ & $100.0 \%$ \\
\hline Africa/Middle East & 7 & $\$ 6,384$ & $2.8 \%$ & $1.0 \%$ \\
\hline Asia/Pacific & 55 & $\$ 11,121$ & $22.0 \%$ & $14.0 \%$ \\
\hline Japan & 31 & $\$ 9,835$ & $12.4 \%$ & $7.0 \%$ \\
\hline Other Asia/Pacific & 24 & $\$ 12,782$ & $9.6 \%$ & $7.0 \%$ \\
\hline Europe & 90 & $\$ 18,840$ & $36.0 \%$ & $38.9 \%$ \\
\hline France & 14 & $\$ 29,279$ & $5.6 \%$ & $9.4 \%$ \\
\hline Germany & 17 & $\$ 27,060$ & $6.8 \%$ & $10.6 \%$ \\
\hline U.K. & 14 & $\$ 19,391$ & $5.6 \%$ & $6.2 \%$ \\
\hline Other Europe & 45 & $\$ 12,316$ & $18.0 \%$ & $12.7 \%$ \\
\hline Latin America & 10 & $\$ 7,757$ & $4.0 \%$ & $1.8 \%$ \\
\hline North America & 88 & $\$ 21,875$ & $35.2 \%$ & $44.2 \%$ \\
\hline U.S. & 79 & $\$ 23,108$ & $31.6 \%$ & $41.9 \%$ \\
\hline Canada & 9 & $\$ 11,052$ & $3.6 \%$ & $2.3 \%$ \\
\hline
\end{tabular}

Results reflect Top 250 retailers headquartered in each region/country
Tesco's operations in China, the United States, and Japan were all treated as discontinued in 2013. Tesco has teamed up with China Resources Enterprise to combine its 134 Chinese stores with CRE's Vanguard unit, which operates almost 3,000 hypermarkets and supermarkets across China and Hong Kong. Tesco will be a minority partner in the joint venture. This move follows decisions to abandon the United States and Japan and focus on investing in its home market.

In line with Carrefour's turnaround plan that includes reducing its presence in Asia and refocusing on core activities, the French retailer sold its 60 percent stake in Carrefour Indonesia to its local partner CT Corp in January 2013. This came shortly after the sale of its Malaysian business to Aeon in November 2012. Carrefour also reorganized its partnership in Turkey, with its Turkish partner becoming the majority shareholder of the joint venture. Metro, Dixons, Delhaize, Makro, Darty, and Dia also disposed of non-core operations in 2013.

\section{Level of globalization by region/country, 2013}

\begin{tabular}{lrrr} 
& $\begin{array}{r}\% \text { retail } \\
\text { revenue } \\
\text { from foreign } \\
\text { operations }\end{array}$ & $\begin{array}{r}\text { Average \# } \\
\text { of countries }\end{array}$ & $\begin{array}{r}\% \text { single- } \\
\text { country } \\
\text { operators }\end{array}$ \\
\hline Top 250 & $24.2 \%$ & 10.2 & $34.8 \%$ \\
\hline Africa/Middle East & $25.1 \%$ & 12.1 & $0.0 \%$ \\
\hline Asia/Pacific & $14.0 \%$ & 5.4 & $43.6 \%$ \\
\hline Japan & $9.4 \%$ & 3.9 & $45.2 \%$ \\
\hline Other Asia/Pacific & $18.9 \%$ & 7.4 & $41.7 \%$ \\
\hline Europe & $38.6 \%$ & 16.2 & $22.2 \%$ \\
\hline France & $43.6 \%$ & 28.6 & $7.1 \%$ \\
\hline Germany & $45.4 \%$ & 15.4 & $5.9 \%$ \\
\hline U.K. & $21.5 \%$ & 16.1 & $21.4 \%$ \\
\hline Latin America & $22.9 \%$ & 2.3 & $40.0 \%$ \\
\hline North America & $14.7 \%$ & 7.8 & $44.3 \%$ \\
\hline U.S. & $15.4 \%$ & 8.5 & $40.5 \%$ \\
\hline
\end{tabular}

Results reflect Top 250 retailers headquartered in each region/country 
In North America, Safeway completed the sale of Canada Safeway to Sobeys, a wholly-owned subsidiary of Empire Company, in November 2013. With Canada Safeway considered a discontinued operation, the company's revenue dropped 19.2 percent. (Note: In March 2014, Safeway announced it had entered into a definitive merger agreement with Albertsons.) Best Buy divested its Best Buy Europe joint venture in June 2013, resulting in a 14.5 percent revenue reduction.

While growth moderated, profitability improved for both regions in 2013. North American retailers reported a 3.5 percent composite net profit margin. For the European region, the composite net profit margin was 3.2 percent. North American retailers made more profitable use of their resources compared with European retailers, generating a 6.8 percent return on assets versus Europe's 4.5 percent.

Among the big three European economies, German retailers saw the strongest top-line growth, albeit a modest 3.5 percent. This compares with 3.3 percent for the French retailers and no growth for the U.K.-based companies on a composite basis. More than one-third (5 of 14 U.K. companies) witnessed declining sales in 2013. On the bottom line, French retailers were the most profitable, posting a composite net profit margin of 3.6 percent.

European retailers are, by far, the most globally active - especially those based in Germany and France where revenue from foreign operations exceeds 40 percent. On average, Europe's Top 250 retailers operated in 16.2 countries in 2013 compared with 10.2 for the Top 250 as a whole and more than twice the North American average of 7.8 countries. While the share of Top 250 retailers that operate outside their home country continues to grow, the North American region maintained the highest percentage of single-country operators (44.3 percent in 2013).
In the Asia/Pacific region, retail revenue grew a robust 9.7 percent in 2013, fueled by Japanese retailers' 10 percent increase in sales. Nearly one-third of Japan's Top 250 retailers (10 companies) enjoyed double-digit sales growth in 2013. Part of the increase - especially late in the fiscal year - was the result of a consumer spending spree that occurred prior to the national sales tax hike that took effect on April 1, 2014.

Acquisitions also played a significant role. Japan's leading electronics and appliance specialty retailer Yamada Denki increased its shareholding to a majority stake in Best Denki in December 2012, making it a consolidated subsidiary and boosting the company's revenue 11.3 percent in 2013. Aeon undertook a series of acquisitions that helped grow the retail giant's 2013 retail revenue by 11.9 percent. Revenue for Bic Camera soared 56.2 percent in 2013 following the acquisition of a controlling stake in its smaller rival Kojima, creating the second-biggest group in Japan's electronics retail industry. Japanese drug store retailers Tsuruha Holdings and Welcia Holdings also made acquisitions during the fiscal year, driving further consolidation in that industry.

The average number of countries with retail operations includes the location of franchised, licensed and joint venture operations in addition to corporate-owned channels of distribution. Where information was available, the number of countries reflects non-store sales channels, such as consumer-oriented e-commerce sites, catalogs and TV shopping programs, as well as store locations. However, for many retailers, specific information about non-store activity was not available.

\section{Sales growth and profitability by region/country ${ }^{1}(\%)$}

20

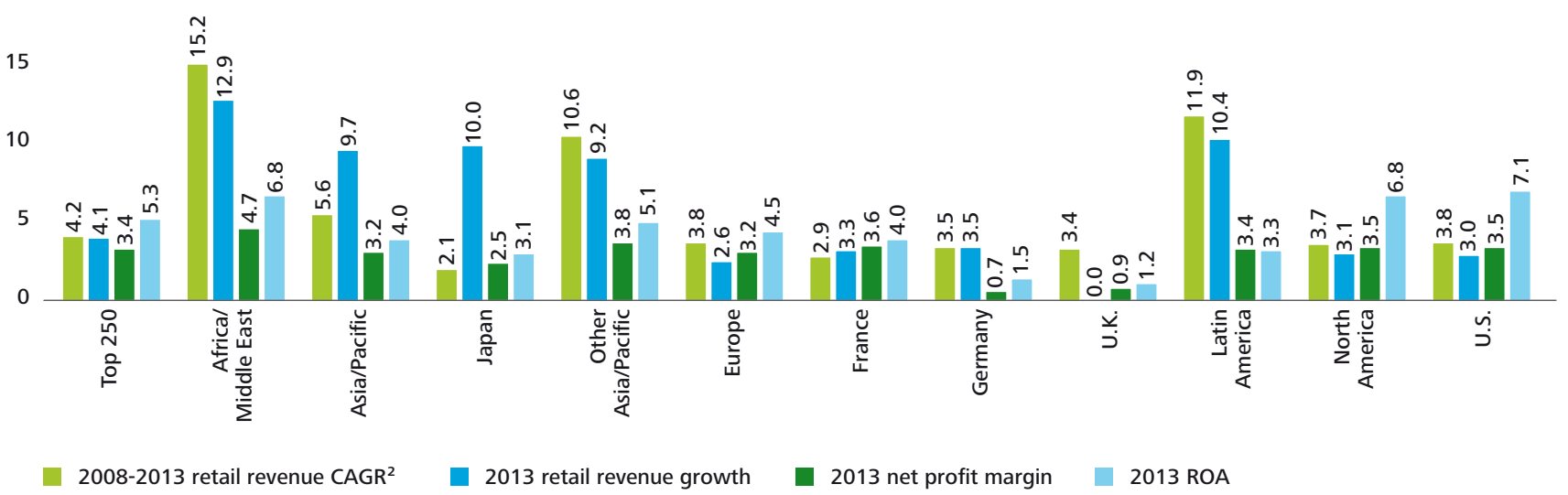

Results reflect Top 250 companies headquartered in each region/country 1 Sales-weighted, currency-adjusted composites 2 Compound annual growth rate

Source: Published company data and Planet Retail 
It should be noted that the continued depreciation of the Japanese yen in 2013 eliminated a number of Japanese retailers who, except for the currency headwinds, would have remained among the Top 250. In 2013, there were 31 Japanese retailers in the Top 250 down from 39 in 2012.

All but one of the 10 Latin American retailers in the Top 250 saw their top-line sales increase in 2013 - seven at a double-digit pace. As a result, composite revenue growth was up 10.4 percent, second only to the Africa/Middle East region. Nevertheless, both sales growth and profitability for the region declined from their 2012 levels, dragged down by Chilean retailer SMU's results as the company implemented a plan to strengthen its financing, including the sale of assets.

Strong growth continued to yield above-average profitability in the Africa/Middle East region in 2013. All seven of the region's Top 250 retailers posted revenue increases, which ranged from 6.5 percent (Pick n Pay Stores) to 21.6 percent (Steinhoff International) and resulted in composite retail revenue growth of 12.9 percent - the highest of any region. The group's composite net profit margin of 4.7 percent also led the industry. The region's six publicly held retailers all operated profitably during the year.

Top 10 retailers by region

Carrefour slipped into the top spot as Europe's largest retailer in 2013, marginally ahead of Schwarz and Tesco. Schwarz moved into second place from third in 2012, the only retailer of the three to post an increase in retail revenue in 2013. Tesco, which had been the top-ranked retailer in 2012, fell to number three as the U.K. retailer offloaded its operations in the United States and Japan and scaled back its ambitions for China. After taking control of Pão de Açúcar in July 2012, Casino rose from eighth place in 2012 to sixth in 2013. Despite these changes to the order, these 10 companies have constituted the region's leader board since 2011.

The top 10 North American retailers, all U.S.-based, did not change in name or ranking in 2013, with Wal-Mart the undisputed leader both regionally and globally. Costco widened its lead over third-ranked Kroger in 2013, having surpassed the region's largest supermarket operator for the first time in 2012. However, Costco may face a challenge in 2014 following Kroger's January 2014 acquisition of Harris Teeter. Best Buy maintained its position as the region's tenth-ranked retailer despite a double-digit sales decline. The U.S. electronics giant sold its 50\% ownership interest in Best Buy Europe to Carphone Warehouse, its former partner, in June 2013.

\begin{tabular}{|c|c|c|c|c|c|}
\hline $\begin{array}{l}\text { Region } \\
\text { rank }\end{array}$ & $\begin{array}{l}\text { Top } \\
250 \\
\text { rank }\end{array}$ & Company & $\begin{array}{l}\text { Country of } \\
\text { origin }\end{array}$ & $\begin{array}{r}2013 \\
\text { retail } \\
\text { revenue } \\
\text { (US\$ mil) }\end{array}$ & $\begin{array}{r}2013 \\
\text { retail } \\
\text { revenue } \\
\text { growth }\end{array}$ \\
\hline \multicolumn{6}{|c|}{ Top 10 European retailers, 2013} \\
\hline 1 & 3 & Carrefour S.A. & France & $\$ 98,688$ & $-2.4 \%$ \\
\hline 2 & 4 & $\begin{array}{l}\text { Schwarz } \\
\text { Unternehmens } \\
\text { Treuhand KG }\end{array}$ & Germany & $\$ 98,662^{\mathrm{e}}$ & $9.5 \%$ \\
\hline 3 & 5 & Tesco PLC & U.K. & $\$ 98,631$ & $-2.0 \%$ \\
\hline 4 & 7 & Metro $\mathrm{Ag}^{1}$ & Germany & $\$ 86,393^{\mathrm{e}}$ & $-2.5 \%$ \\
\hline 5 & 8 & $\begin{array}{l}\text { Aldi Einkauf GmbH } \\
\text { \& Co. oHG }\end{array}$ & Germany & $\$ 81,090^{\mathrm{e}}$ & $4.7 \%$ \\
\hline 6 & 13 & $\begin{array}{l}\text { Casino Guichard- } \\
\text { Perrachon S.A. }\end{array}$ & France & $\$ 63,468^{* *}$ & $15.1 \%$ \\
\hline 7 & 14 & Groupe Auchan SA & France & $\$ 62,444$ & $2.4 \%$ \\
\hline 8 & 16 & $\begin{array}{l}\text { Edeka Zentrale AG } \\
\text { \& Co. KG }\end{array}$ & Germany & $\$ 59,704^{* *}$ & $3.3 \%$ \\
\hline 9 & 21 & Rewe Combine & Germany & $\$ 51,109^{* *}$ & $1.0 \%$ \\
\hline 10 & 23 & $\begin{array}{l}\text { Centres Distributeurs } \\
\text { E. Leclerc }\end{array}$ & France & $\$ 47,671^{\mathrm{e}^{* *}}$ & $4.1 \%$ \\
\hline
\end{tabular}

\begin{tabular}{lcllll}
\hline \multicolumn{7}{l}{ Top 10 } & North & American retailers, 2013 & & \\
\hline 1 & 1 & Wal-Mart Stores, Inc. & U.S. & $\$ 476,294$ & $1.5 \%$ \\
\hline 2 & 2 & $\begin{array}{l}\text { Costco Wholesale } \\
\text { Corporation }\end{array}$ & U.S. & $\$ 105,156$ & $6.1 \%$ \\
\hline 3 & 6 & The Kroger Co. & U.S. & $\$ 98,375$ & $1.7 \%$ \\
\hline 4 & 9 & The Home Depot, Inc. & U.S. & $\$ 78,812$ & $5.4 \%$ \\
\hline 5 & 10 & Target Corporation & U.S. & $\$ 72,596$ & $0.9 \%$ \\
\hline 6 & 11 & Walgreen Co. & U.S. & $\$ 72,217$ & $0.8 \%$ \\
\hline 7 & 12 & $\begin{array}{l}\text { CVS Caremark } \\
\text { Corporation }\end{array}$ & U.S. & $\$ 65,618$ & $3.1 \%$ \\
\hline 8 & 15 & Amazon.com, Inc. & U.S. & $\$ 60,903$ & $17.7 \%$ \\
\hline 9 & 20 & $\begin{array}{l}\text { Lowe's Companies, } \\
\text { Inc. }\end{array}$ & U.S. & $\$ 53,417$ & $5.7 \%$ \\
\hline 10 & 25 & Best Buy Co., Inc. & U.S. & $\$ 42,410$ & $-14.5 \%$ \\
\hline
\end{tabular}

\footnotetext{
${ }^{1}$ Metro changed its fiscal year from end of December to end of September. Fiscal 2013 revenue reported here includes the 9 months ended 30 September plus the 3 months ended 31 December.

e = estimate

** Revenue includes wholesale and retail sales
} 
A recent series of acquisitions kept Japan's Aeon in the lead as the largest retailer in the Asia/Pacific region. Following the November 2012 acquisition of Carrefour Malaysia, Aeon bought a 50 percent stake in Tesco Japan in January 2013. In March, the company acquired Peacock Stores, a Japanese supermarket chain, from J. Front Retailing. In September 2014, Aeon agreed to acquire the remaining shares in struggling supermarket operator Daiei that it does not already own, making it a wholly owned subsidiary in 2015. In October 2014, it made an offer to acquire the remaining stake in supermarket operator Maruetsu. Also in October, the company offered to acquire an additional stake in Welcia Holdings, a Japanese drug store chain that just made its debut among the Top 250 (see Top 250 newcomers, p.29. The transaction will increase Aeon's shareholding to 50.01 percent and is expected to create the largest drug store retailer in Japan.

There were only two minor changes to the Asia/Pacific region's top 10 in 2013. A.S. Watson ranked ahead of Yamada Denki due to the relative strength of the Hong Kong dollar versus the yen in the dollar-denominated ranking. Gome Home Appliance Group rejoined the leader board following a one-year absence. The retailer fell out of the top 10 in 2012 when its revenue and store count both dropped significantly, the result of slower economic growth in China and competition from online channels.

In 2013, the Latin American region included 10 Top 250 companies compared with nine in 2012. Magazine Luiza, occupying tenth place, is Brazil's second-largest retailer. Except for this newcomer, the list is virtually unchanged from the prior year. Mexico's Coppel, a retailer of electronics and household goods to low-income consumers, moved ahead of Brazil's Lojas Americanas, although both retailers reported strong, double-digit sales growth in 2013. The depreciation of the Brazilian real in 2013 was responsible for the change in the companies' relative ranking.

The Africa/Middle East region continued to be represented by seven retailers in 2013. Five are based in South Africa, including top-ranked Shoprite Holdings. The country's largest food retailer, Shoprite operates in 15 markets across sub-Saharan Africa. Expansion continues mainly organically at home and abroad. Fast-growing furniture retailer Steinhoff International was the region's second-largest retailer in 2013. Also based in South Africa, the company operates in 26 markets, primarily in Africa and Europe. In November 2013, Steinhoff acquired the kika-Leiner Group, a furniture retailer with 73 shops in Austria, Central and Eastern Europe. With annual sales of approximately 1.2 billion euros, the acquisition will enable Steinhoff to challenge Shoprite for the top spot in the future.

\begin{tabular}{|c|c|c|c|c|c|}
\hline $\begin{array}{l}\text { Region } \\
\text { rank }\end{array}$ & $\begin{array}{l}\text { Top } \\
250 \\
\text { rank }\end{array}$ & Company & $\begin{array}{l}\text { Country of } \\
\text { origin }\end{array}$ & $\begin{array}{r}2013 \\
\text { retail } \\
\text { revenue } \\
\text { (US\$ mil) }\end{array}$ & $\begin{array}{r}2013 \\
\text { retail } \\
\text { revenue } \\
\text { growth }\end{array}$ \\
\hline \multicolumn{6}{|c|}{ Top 10 Asia/Pacific retailers, 2013} \\
\hline 1 & 17 & Aeon Co., Ltd. & Japan & $\$ 57,986^{* *}$ & $11.9 \%$ \\
\hline 2 & 18 & Woolworths Limited & Australia & $\$ 54,457$ & $3.9 \%$ \\
\hline 3 & 19 & $\begin{array}{l}\text { Seven \& i Holdings } \\
\text { Co., Ltd. }\end{array}$ & Japan & $\$ 54,258^{* *}$ & $13.3 \%$ \\
\hline 4 & 22 & Wesfarmers Limited & Australia & $\$ 50,711$ & $4.6 \%$ \\
\hline 5 & 40 & $\begin{array}{l}\text { Lotte Shopping Co., } \\
\text { Ltd. }\end{array}$ & S. Korea & $\$ 24,601$ & $13.4 \%$ \\
\hline 6 & 51 & A.S. Watson Group & $\begin{array}{l}\text { Hong } \\
\text { Kong SAR }\end{array}$ & $\$ 19,230^{* *}$ & $0.4 \%$ \\
\hline 7 & 53 & $\begin{array}{l}\text { Yamada Denki Co., } \\
\text { Ltd. }\end{array}$ & Japan & $\$ 18,921^{* *}$ & $11.3 \%$ \\
\hline 8 & 59 & $\begin{array}{l}\text { Suning Commerce } \\
\text { Group Co., Ltd. }\end{array}$ & China & $\$ 16,616$ & $4.6 \%$ \\
\hline 9 & 69 & $\begin{array}{l}\text { Gome Home } \\
\text { Appliance Group }\end{array}$ & China & $\$ 13,441^{\mathrm{e}}$ & $9.6 \%$ \\
\hline 10 & 74 & $\begin{array}{l}\text { Isetan Mitsukoshi } \\
\text { Holdings Ltd. }\end{array}$ & Japan & $\$ 12,856$ & $6.7 \%$ \\
\hline
\end{tabular}

\begin{tabular}{|c|c|c|c|c|c|}
\hline \multicolumn{6}{|c|}{ Top 10 Latin American retailers, 2013} \\
\hline 1 & 47 & Cencosud S.A. & Chile & $\$ 19,855$ & $13.1 \%$ \\
\hline 2 & 88 & S.A.C.I. Falabella & Chile & $\$ 11,377$ & $13.0 \%$ \\
\hline 3 & 127 & $\begin{array}{l}\text { Organización Soriana, } \\
\text { S.A.B. de C.V. }\end{array}$ & Mexico & $\$ 8,240^{* *}$ & $0.4 \%$ \\
\hline 4 & 134 & $\begin{array}{l}\text { FEMSA Comercio, S.A. } \\
\text { de C.V. }\end{array}$ & Mexico & $\$ 7,655$ & $12.9 \%$ \\
\hline 5 & 149 & Coppel S.A. de C.V. & Mexico & $\$ 6,304$ & $17.0 \%$ \\
\hline 6 & 150 & Lojas Americanas S.A. & Brazil & $\$ 6,247$ & $18.2 \%$ \\
\hline 7 & 176 & $\begin{array}{l}\text { Grupo Comercial } \\
\text { Chedraui, S.A.B. de } \\
\text { C.V. }\end{array}$ & Mexico & $\$ 5,157$ & $3.8 \%$ \\
\hline 8 & 177 & $\begin{array}{l}\text { El Puerto de Liverpool, } \\
\text { S.A.B. de C.V. }\end{array}$ & Mexico & $\$ 5,156$ & $11.8 \%$ \\
\hline 9 & 241 & SMU S.A. & Chile & $\$ 3,823^{* *}$ & $-9.2 \%$ \\
\hline 10 & 247 & Magazine Luiza SA & Brazil & $\$ 3,758$ & $14.5 \%$ \\
\hline
\end{tabular}

\begin{tabular}{lcllll}
\hline \multicolumn{5}{l}{ Top Africa/Middle East retailers, 2013} \\
\hline 1 & 107 & Shoprite Holdings Ltd. & S. Africa & $\$ 9,869^{* *}$ & $10.2 \%$ \\
\hline 2 & 128 & $\begin{array}{l}\text { Steinhoff International } \\
\text { Holdings Ltd. Africa }\end{array}$ & $\$ 8,217$ & $21.6 \%$ \\
\hline 3 & 148 & $\begin{array}{l}\text { Pick n Pay Stores } \\
\text { Limited }\end{array}$ & S. Africa & $\$ 6,351$ & $6.5 \%$ \\
\hline 4 & 151 & $\begin{array}{l}\text { BiM Birleşik } \\
\text { Mağazalar A.Ş. }\end{array}$ & Turkey & $\$ 6,242$ & $19.6 \%$ \\
\hline 5 & 174 & $\begin{array}{l}\text { The SPAR Group } \\
\text { Limited }\end{array}$ & S. Africa & $\$ 5,175^{* *}$ & $9.7 \%$ \\
\hline 6 & 183 & $\begin{array}{l}\text { Emke Group/Lulu } \\
\text { Group International }\end{array}$ & UAE & $\$ 5,000^{\text {e }}$ & $9.6 \%$ \\
\hline 7 & 239 & $\begin{array}{l}\text { Woolworths Holdings } \\
\text { Limited }\end{array}$ & S. Africa & $\$ 3,834$ & $12.7 \%$ \\
\hline
\end{tabular}

e = estimate

** Revenue includes wholesale and retail sales 


\title{
Global Powers of Retailing product sector analysis
}

\author{
The Global Powers of Retailing analyzes retail performance by primary retail product sector as \\ well as by geography. Four sectors are used for analysis: Apparel \& Accessories, Fast-Moving \\ Consumer Goods, Hardlines \& Leisure Goods, and Diversified. A company is assigned to one of \\ three specific product sectors if at least half of its sales are derived from that broadly defined \\ product category. If none of the three specific product sectors account for at least 50 percent \\ of a company's sales, it is considered to be diversified.
}

Softlines and hardlines retailers outpace fast-moving consumer goods in 2013

Retailers of food and other fast-moving consumer goods (FMCG) continue to dominate the Top 250 in number and size of companies. However, unlike in 2011 and 2012 when this sector enjoyed stronger growth than the other product groups, in 2013, it took a back seat to both softlines and hardlines retailers. Still, composite retail revenue grew 4.0 percent, and profitability remained solid at 2.8 percent. In the aggregate, the 132 FMCG retailers accounted for over half of Top 250 companies and more than two-thirds of Top 250 retail revenue.

Despite their large size - retailers of fast-moving consumer goods generated average 2013 retail revenue of more than $\$ 22$ billion - companies in this sector are the least geographically dispersed, concentrating their operations in an average of 4.9 countries. More than 40 percent continue to operate only within their domestic borders. Nevertheless, foreign operations generated a sizeable $23.2 \%$ share of FMCG retailers' combined sales. And, it should be noted, the overall sector results disguise the global expansion activity of several of the largest companies that operate in dozens of countries and derive the majority of their sales from foreign operations.

The high-margin apparel \& accessories sector was the fastestgrowing product group in 2013 with 5.8 percent composite revenue growth.
Product sector profiles, 2013

\begin{tabular}{lrrrr} 
& $\begin{array}{r}\text { \# } \\
\text { companies }\end{array}$ & $\begin{array}{r}\text { Average } \\
\text { retail revenue } \\
\text { (US\$mil) }\end{array}$ & $\begin{array}{r}\text { Share of } \\
\text { Top 250 } \\
\text { Companies }\end{array}$ & $\begin{array}{r}\text { Share of } \\
\text { Top 250 } \\
\text { Revenue }\end{array}$ \\
\hline Top 250 & 250 & $\$ 17,418$ & $100.0 \%$ & $100.0 \%$ \\
\hline $\begin{array}{l}\text { Apparel \& } \\
\text { Accessories }\end{array}$ & 44 & $\$ 9,145$ & $17.6 \%$ & $9.2 \%$ \\
\hline $\begin{array}{l}\text { Fast-Moving } \\
\text { Consumer } \\
\text { Goods }\end{array}$ & 132 & $\$ 22,269$ & $52.8 \%$ & $67.5 \%$ \\
\hline $\begin{array}{l}\text { Hardlines \& } \\
\text { Leisure Goods }\end{array}$ & 52 & $\$ 12,620$ & $20.8 \%$ & $15.1 \%$ \\
\hline Diversified & 22 & $\$ 16,200$ & $8.8 \%$ & $8.2 \%$ \\
\hline
\end{tabular}

Source: Published company data and Planet Retail

Sales growth and profitability by product sector ${ }^{1}(\%)$

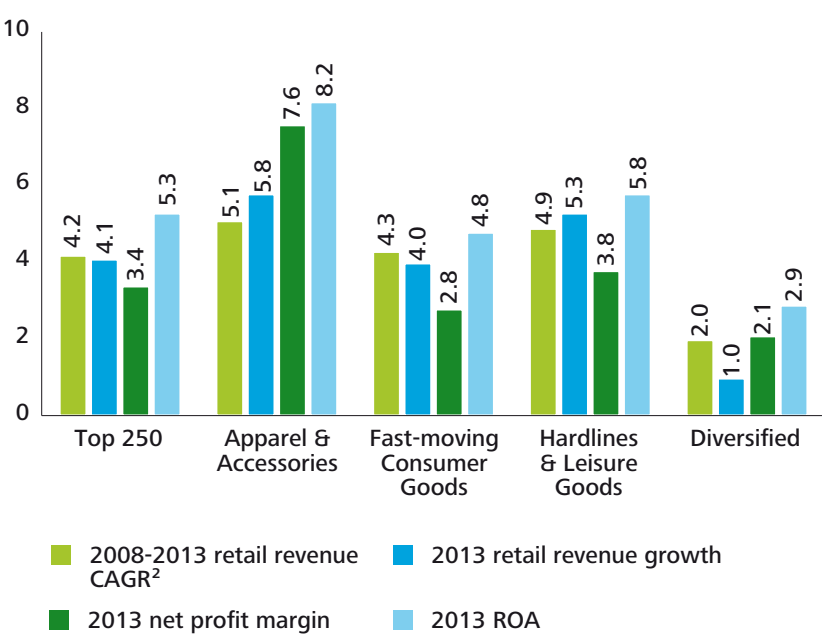

1 Sales-weighted, currency-adjusted composites

2 Compound annual growth rate

Source: Published company data and Planet Retail 
This group of companies - although relatively small in size with average retail revenue of $\$ 9.1$ billion - has the distinction of being the most global. Thirty-seven of the 44 Top 250 apparel $\&$ accessories retailers operated internationally in 2013. On average, retailers in this product sector have expanded their operations to more than 27 countries around the globe and generated nearly one-third of their revenues outside their home country.

International expansion also continues to be an important growth strategy for many retailers of hardlines \& leisure goods, particularly for European companies, many of which have expanded into a number of neighboring countries within their region. In 2013, the 52 companies in this sector operated in 8.7 countries, on average. As a group, they generated one-quarter of their total retail revenue from foreign operations. In 2013, this sector saw an improvement in both top-line sales and bottom-line profits. Composite retail revenue grew a healthy 5.3 percent. The group produced a composite net profit margin of 3.8 percent.

Diversified retailers sell a broad product offering and often operate a range of formats including department stores, hypermarkets, general merchandise discount stores, specialty stores, and non-store channels. This group was represented by 22 companies in 2013. Including the likes of Germany's Metro group, Target and Sears Holdings in the United States, and British retailer Marks \& Spencer, the average size of the companies in this group was more than $\$ 16$ billion.

Historically, the diversified group has underperformed compared with the three specific product sectors, and 2013 was no exception. Composite revenue growth was an anemic 1.0\%. The group's composite net profit margin, at $2.1 \%$, was also subpar. However, diversified companies from emerging growth markets - South Korea's Lotte Shopping Co., Chile's Falabella, Brazil's Lojas Americanas, Thailand's Central Retail Corp., Philippines' SM Investments Corp., and South Africa's Woolworths Holdings greatly outperformed the group's composite results.

Level of globalization by product sector, 2013

\begin{tabular}{lrrr} 
& $\begin{array}{r}\text { \% retail revenue } \\
\text { from foreign } \\
\text { operations }\end{array}$ & $\begin{array}{r}\text { Average \# } \\
\text { countries }\end{array}$ & $\begin{array}{r}\text { country } \\
\text { coperators }\end{array}$ \\
\hline Top 250 & $24.2 \%$ & 10.2 & $34.8 \%$ \\
\hline Apparel \& Accessories & $31.6 \%$ & 27.3 & $15.9 \%$ \\
\hline $\begin{array}{l}\text { Fast-Moving Consumer } \\
\text { Goods }\end{array}$ & $23.2 \%$ & 4.9 & $41.7 \%$ \\
\hline Hardlines \& Leisure Goods & $25.2 \%$ & 8.7 & $36.5 \%$ \\
\hline Diversified & $23.5 \%$ & 11.2 & $27.3 \%$ \\
\hline
\end{tabular}

Source: Published company data and Planet Retail
Top 10 retailers by product sector

The eight largest fast-moving consumer goods retailers have been ranked among the sector's top 10 since 2006 when Walgreen first joined the leader board. CVS moved into the FMCG top 10 in 2009 following the acquisition of Longs Drug Stores in October 2008. In 2013, the sector's tenth-ranked company, Casino, rose to 13th place overall among the Top 250 from 20th place in 2012, mainly reflecting the full consolidation of Pão de Açúcar (now called GPA) from July 2012 and Monoprix from April 2013. Japan's Aeon was bumped from the FMCG top 10 by Casino despite a double-digit sales increase, a result of the weak yen. Other noteworthy changes are Costco's continued ascent into second place behind Wal-Mart and the battle for third place among Carrefour, Schwarz, Tesco, and Kroger - all with 2013 retail revenue of $\$ 98+$ billion.

\begin{tabular}{|c|c|c|c|c|c|}
\hline Sector & $\begin{array}{l}\text { Top } \\
250\end{array}$ & & Country of & $\begin{array}{r}2013 \\
\text { retail } \\
\text { revenue }\end{array}$ & $\begin{array}{r}2013 \\
\text { retail } \\
\text { revenue }\end{array}$ \\
\hline rank & rank & Company & origin & (US\$ mil) & growth \\
\hline
\end{tabular}

Top 10 fast-moving consumer goods retailers, 2013

\begin{tabular}{|c|c|c|c|c|c|}
\hline 1 & 1 & Wal-Mart Stores, Inc. & U.S. & $\$ 476,294$ & $1.5 \%$ \\
\hline 2 & 2 & $\begin{array}{l}\text { Costco Wholesale } \\
\text { Corporation }\end{array}$ & U.S. & $\$ 105,156$ & $6.1 \%$ \\
\hline 3 & 3 & Carrefour S.A. & France & $\$ 98,688$ & $-2.4 \%$ \\
\hline 4 & 4 & $\begin{array}{l}\text { Schwarz } \\
\text { Unternehmens } \\
\text { Treuhand KG }\end{array}$ & Germany & $\$ 98,662^{\mathrm{e}}$ & $9.5 \%$ \\
\hline 5 & 5 & Tesco PLC & U.K. & $\$ 98,631$ & $-2.0 \%$ \\
\hline 6 & 6 & The Kroger Co. & U.S. & $\$ 98,375$ & $1.7 \%$ \\
\hline 7 & 8 & $\begin{array}{l}\text { Aldi Einkauf } \mathrm{GmbH} \& \\
\text { Co. oHG }\end{array}$ & Germany & $\$ 81,090^{e}$ & $4.7 \%$ \\
\hline 8 & 11 & Walgreen Co. & U.S. & $\$ 72,217$ & $0.8 \%$ \\
\hline 9 & 12 & $\begin{array}{l}\text { CVS Caremark } \\
\text { Corporation }\end{array}$ & U.S. & $\$ 65,618$ & $3.1 \%$ \\
\hline 10 & 13 & $\begin{array}{l}\text { Casino Guichard- } \\
\text { Perrachon S.A. }\end{array}$ & France & $\$ 63,468^{* *}$ & $15.1 \%$ \\
\hline
\end{tabular}

e = estimate

** Revenue includes wholesale and retail sales 
Macy's held onto its lead as the top apparel $\&$ accessories retailer - but not by much, with TJX right on its heels. H\&M moved into fifth place ahead of Kohl's whose sales declined in 2013. The biggest changes, however, took place at the bottom of the list. Sales soared in 2013 for Japan's Fast Retailing, best known for its UNIQLO casualwear brand, boosting the fashion retailer into the sector's top 10 for the first time. Sustained growth also propelled Nordstrom back into the top 10, while J.C. Penney, once one of the sector's largest players, fell out of contention altogether in 2013 as sales declined for the third consecutive year. Japanese department store retailer J. Front Retailing dropped out of the top 10 in 2013, another victim of the depreciating yen.

The five largest retailers on the 2013 hardlines \& leisure goods top 10 were the same as the year before with Home Depot as the frontrunner. Amazon remained as the sector's second biggest retailer despite a change in how its retail revenue was calculated for purposes of the Top 250 ranking. For the first time in 2013, retail revenue was defined as the company's net product sales where Amazon is the seller of record. It now excludes the sale of "services," which represent third-party seller fees and digital content subscriptions, as well as non-retail activities such as Amazon Web Services, advertising services, and its co-branded credit card agreements. Fourth-ranked Best Buy's lead over fifthranked IKEA continued to narrow following back-to-back years of declining sales for Best Buy. As already noted, Best Buy sold its $50 \%$ ownership interest in Best Buy Europe to Carphone Warehouse in June 2103. In August 2014, Carphone Warehouse and Dixons Retail merged to create Dixons Carphone, a contender for top 10 status in the future. On the bottom half of the list, the companies remained the same as in 2012 although in slightly different order.

The top 10 diversified retailers did not change in 2013 except at the very bottom of the list where Finland's Kesko - a multi-format food and general merchandise retailer - ranked tenth, displacing Beisia Group, which fell out of contention due to the yen's unfavorable currency exchange rate.

Metro maintained its lead as the largest diversified retailer even though sales declined as the company continued to reshape the group for the future. It should be noted that the ranking is based on annualized revenue for Metro Group, which changed its fiscal year from the end of December to the end of September in 2013.

\begin{tabular}{|c|c|c|c|c|c|}
\hline $\begin{array}{l}\text { Sector } \\
\text { rank }\end{array}$ & $\begin{array}{l}\text { Top } \\
250 \\
\text { rank }\end{array}$ & Company & $\begin{array}{l}\text { Country of } \\
\text { origin }\end{array}$ & $\begin{array}{r}2013 \\
\text { retail } \\
\text { revenue } \\
\text { (US\$ mil) }\end{array}$ & $\begin{array}{r}2013 \\
\text { retail } \\
\text { revenue } \\
\text { growth }\end{array}$ \\
\hline \multicolumn{6}{|c|}{ Top 10 apparel \& accessories retailers, 2013} \\
\hline 1 & 34 & Macy's, Inc. & U.S. & $\$ 27,931^{* *}$ & $0.9 \%$ \\
\hline 2 & 36 & $\begin{array}{l}\text { The TJX Companies, } \\
\text { Inc. }\end{array}$ & U.S. & $\$ 27,423$ & $6.0 \%$ \\
\hline 3 & 41 & $\begin{array}{l}\text { LVMH Moët } \\
\text { Hennessy- } \\
\text { Louis Vuitton S.A. }\end{array}$ & France & $\$ 24,392^{\mathrm{e}}$ & $3.7 \%$ \\
\hline 4 & 44 & Inditex, S.A. & Spain & $\$ 22,265^{* *}$ & $4.9 \%$ \\
\hline 5 & 49 & $\begin{array}{l}\mathrm{H} \& \mathrm{M} \text { Hennes \& } \\
\text { Mauritz } \mathrm{AB}\end{array}$ & Sweden & $\$ 19,729$ & $6.4 \%$ \\
\hline 6 & 52 & Kohl's Corporation & U.S. & $\$ 19,031$ & $-1.3 \%$ \\
\hline 7 & 61 & The Gap, Inc. & U.S. & $\$ 16,148$ & $3.2 \%$ \\
\hline 8 & 74 & $\begin{array}{l}\text { Isetan Mitsukoshi } \\
\text { Holdings Ltd. }\end{array}$ & Japan & $\$ 12,856$ & $6.7 \%$ \\
\hline 9 & 76 & $\begin{array}{l}\text { Fast Retailing Co., } \\
\text { Ltd. }\end{array}$ & Japan & $\$ 12,639^{* *}$ & $23.2 \%$ \\
\hline 10 & 80 & Nordstrom, Inc. & U.S. & $\$ 12,166$ & $3.4 \%$ \\
\hline
\end{tabular}

\begin{tabular}{lclllr}
\hline \multicolumn{7}{l}{ Top 10 hardlines \& leisure goods retailers, 2013} \\
\hline 1 & 9 & The Home Depot, Inc. U.S. & $\$ 78,812$ & $5.4 \%$ \\
\hline 2 & 15 & Amazon.com, Inc. & U.S. & $\$ 60,903$ & $17.7 \%$ \\
\hline 3 & 20 & $\begin{array}{l}\text { Lowe's Companies, } \\
\text { Inc. }\end{array}$ & U.S. & $\$ 53,417$ & $5.7 \%$ \\
\hline 4 & 25 & Best Buy Co., Inc. & U.S. & $\$ 42,410$ & $-14.5 \%$ \\
\hline 5 & 28 & $\begin{array}{l}\text { The IKEA Group } \\
\text { (INGKA Holding B.V.) }\end{array}$ & Netherlands & $\$ 36,495$ & $3.3 \%$ \\
\hline 6 & 46 & $\begin{array}{l}\text { Apple Inc./Apple } \\
\text { Retail Stores }\end{array}$ & U.S. & $\$ 20,228$ & $7.4 \%$ \\
\hline 7 & 53 & $\begin{array}{l}\text { Yamada Denki Co., } \\
\text { Ltd. }\end{array}$ & Japan & $\$ 18,921^{* *}$ & $11.3 \%$ \\
\hline 8 & 54 & $\begin{array}{l}\text { Groupe Adeo SA } \\
\text { Kingfisher plc }\end{array}$ & France & $\$ 18,197^{\mathrm{e}^{* *}}$ & $5.3 \%$ \\
\hline 9 & 57 & U.K. & $\$ 17,454$ & $5.2 \%$ \\
\hline 10 & 59 & $\begin{array}{l}\text { Suning Commerce } \\
\text { Group Co., Ltd. }\end{array}$ & China & $\$ 16,616$ & $4.6 \%$ \\
\hline
\end{tabular}

\begin{tabular}{lllllr}
\hline \multicolumn{7}{l}{ Top 10 diversified retailers, 2013 } & & \\
\hline 1 & 7 & ${\text { Metro } \mathbf{A g}^{1}}$ & Germany & $\$ 86,393^{\mathrm{e}}$ & $-2.5 \%$ \\
\hline 2 & 10 & Target Corporation & U.S. & $\$ 72,596$ & $0.9 \%$ \\
\hline 3 & 29 & Sears Holdings Corp. & U.S. & $\$ 36,188$ & $-9.2 \%$ \\
\hline 4 & 40 & $\begin{array}{l}\text { Lotte Shopping Co., } \\
\text { Ltd. }\end{array}$ & S. Korea & $\$ 24,601$ & $13.4 \%$ \\
\hline 5 & 60 & $\begin{array}{l}\text { Marks and Spencer } \\
\text { Group plc }\end{array}$ & U.K. & $\$ 16,391$ & $2.8 \%$ \\
\hline 6 & 66 & El Corte Inglés, S.A. & Spain & $\$ 14,789$ & $-2.4 \%$ \\
\hline 7 & 70 & $\begin{array}{l}\text { Otto (GmbH \& Co KG) } \\
\text { Germany }\end{array}$ & $\$ 13,355$ & $-0.4 \%$ \\
\hline 8 & 88 & S.A.C.I. Falabella & Chile & $\$ 11,377$ & $13.0 \%$ \\
\hline 9 & 99 & $\begin{array}{l}\text { Liberty Interactive } \\
\text { Corporation }\end{array}$ & U.S. & $\$ 10,307$ & $2.9 \%$ \\
\hline 10 & 121 & Kesko Corporation & Finland & $\$ 8,776^{* *}$ & $-7.2 \%$ \\
\hline
\end{tabular}

\footnotetext{
${ }^{1}$ Metro changed its fiscal year from end of December to end of September. Fiscal 2013 revenue reported here includes the 9 months ended 30 September plus the 3 months ended 31 December.

e = estimate

** Revenue includes wholesale and retail sales
} 


\section{Top 250 newcomers}

Top 250 newcomers, 2013

\begin{tabular}{|c|c|c|c|c|}
\hline $\begin{array}{l}\text { Top } 250 \\
\text { rank }\end{array}$ & Name of company & $\begin{array}{l}\text { Country } \\
\text { of origin }\end{array}$ & Dominant format & $\begin{array}{r}2013 \text { retail } \\
\text { revenue } \\
\text { growth }\end{array}$ \\
\hline 104 & Chow Tai Fook Jewellery Group Limited & Hong Kong SAR & Other Specialty & $9,979.3^{\star \star}$ \\
\hline 182 & Foodstuffs North Island Ltd & New Zealand & Hypermarket/Supercenter/Superstore & $5,021 \cdot 2^{\mathrm{e}^{* *}}$ \\
\hline 189 & Yonghui Superstores Co., Ltd. & China & Hypermarket/Supercenter/Superstore & $4,733.1$ \\
\hline 190 & Ascena Retail Group, Inc. & U.S. & Apparel/Footwear Specialty & $4,714.9$ \\
\hline 196 & Lenta Group & Russia & Hypermarket/Supercenter/Superstore & $4,538.6$ \\
\hline 206 & O’Key Group S.A. & Russia & Hypermarket/Supercenter/Superstore & $4,330.1$ \\
\hline 214 & SM Investments Corporation & Philippines & Department Store & $4,275.8$ \\
\hline 224 & Forever 21, Inc. & U.S. & Apparel/Footwear Specialty & $4,000.0^{\mathrm{e}}$ \\
\hline 225 & PRADA Group & Italy & Apparel/Footwear Specialty & $3,989.3$ \\
\hline 227 & Gruppo Eurospin & Italy & Discount Store & $3,984.8^{\mathrm{e}^{* *}}$ \\
\hline 232 & Academy Sports + Outdoors & U.S. & Other Specialty & $3,900.0^{\mathrm{e}}$ \\
\hline 243 & Reinalt-Thomas Corporation (dba Discount Tire/America's Tire) & U.S. & Other Specialty & $3,800.0^{\mathrm{e}}$ \\
\hline 244 & Tiffany \& Co. & U.S. & Other Specialty & $3,800.0^{\mathrm{e}}$ \\
\hline 245 & Ralph Lauren Corporation & U.S. & Apparel/Footwear Specialty & $3,798.0$ \\
\hline 247 & Magazine Luiza SA & Brazil & Electronics Specialty & $3,757.6$ \\
\hline 248 & HTM-Group & France & Electronics Specialty & $3,705.8^{e}$ \\
\hline 249 & Welcia Holdings Co., Ltd. & Japan & Drug Store/Pharmacy & $3,705.1$ \\
\hline
\end{tabular}

e = estimate $\quad * *$ Revenue includes wholesale and retail sales

Source: Published company data and Planet Retail

Seventeen retailers joined the ranks of the Top 250 for the first time in 2013. The exclusion of seven gasoline station/convenience store companies in 2013 made way for many of the newcomers on the list.

Chow Tai Fook Jewellery Group, ranked 104th, is a leading jeweler in Mainland China, Hong Kong, and Macau. The company has an extensive retail network with over 2,100 points of sale spanning more than 480 cities in Greater China, Singapore, Malaysia, and South Korea as well as a strong presence on various e-tail platforms.

Four of the newcomers operate hypermarkets and supermarkets. Foodstuffs North Island Ltd. was created on 1 September 2013 through the merger of Foodstuffs Wellington and Foodstuffs Auckland, two of New Zealand's leading grocery retailer and wholesaler cooperatives. Yonghui Superstores, the second-fastestgrowing retailer over the past five years (see Fastest 50, p. G31), is aggressively expanding across China. Lenta Group and O'Key Group are leading hypermarket operators in Russia.

Six U.S. retailers moved into the Top 250 for the first time in 2013. Ascena Retail Group, formerly The Dress Barn, acquired Charming Shoppes - a retailer specializing in women's plus-size apparel - in June 2012, which boosted the company into the Top 250 in 2013 following a full year of consolidation.
Successive years of solid growth propelled fashion retailer Forever 21; full-line sporting goods chain Academy Sports; tire and wheel retailer Discount Tire; Tiffany, a retailer of fine jewelry and other luxury items; and Ralph Lauren, designer and marketer of apparel and other premium lifestyle products, into the Top 250 for the first time in 2013.

Two Italian retailers targeting opposite ends of the socioeconomic spectrum made their Top 250 debut: Prada Group, a brand synonymous with luxury, and economy chain Gruppo Eurospin, the leading discount store banner in Italy. Two consumer electronics retailers are also new to the list. Magazine Luiza is one of Brazil's largest electronics and furniture retailers. France's HTM-Group operates a network of consumer electronics retail chains.

Other Top 250 newcomers in 2013 include SM Investments, a dominant retail player in its home market of the Philippines. The company has a strong presence with its multi-format operations consisting of department stores, supermarkets, and hypermarkets. Welcia Holdings is Japan's only newcomer in 2013. However, its tenure as a Top 250 retailer will likely be short lived. In April 2014, Aeon announced that, by February 2015, it plans to make the drug store operator its consolidated subsidiary, increasing its stake to more than 50 percent from its current 29.4 percent. 


\section{Global Powers of Retailing Fastest 50}

\section{The Fastest 50 is based on compound annual revenue growth over a five-year period. Fastest 50 companies that were also among the 50 fastest-growing retailers in 2013 make up an even more elite group. These retailers are designated in italicized bold type on the list.}

Chinese online retailer JD.com heads Fastest 50

Retailers headquartered in emerging markets accounted for the majority of the Fastest 50 in 2013 (29 companies). In particular, Chinese retailers (including Hong-Kong-based companies) and Russian retailers are well represented among the 50 fastestgrowing retailers over the 2008 through 2013 period, with nine and six companies, respectively.

Thirty-three Fastest 50 companies in 2013 were also among the Fastest 50 in 2012, as they continued to be propelled by acquisitions made over the five-year period and/or robust organic growth. Eleven companies on the list are 2013 Top 250 newcomers.

JD.com, the largest online direct sales company in China was the fastest-growing Top 250 retailer with compound annual growth of 123.6 percent. The company was founded in 2004 and went public in May 2014. In March 2013, Albertson's LLC - third on the list - acquired 870 Albertsons, Acme, Jewel-Osco, Shaw's and Star Market sites from Supervalu Inc., which had owned them since 2006 when Albertsons Inc. was broken up and sold. As a result, Albertson's generated the fastest year-over-year growth in 2013 489.7 percent.
Chilean supermarket operator SMU has the dubious distinction of being the only company in the Fastest 50 to report a decline in revenue in 2013. Although the company's compound annual growth rate ranked among the Fastest 50 due to a flurry of acquisitions over the five-year period, 2013 saw major financial restructuring as SMU closed a number of outlets and put three chains up for sale.

As a group, the 50 fastest-growing retailers increased retail revenue at a compound annual rate of 20.6 percent between 2008 and 2013, nearly five times the rate of the Top 250 overall. Fastest 50 retailers also significantly outperformed the Top 250 on the bottom line with a composite net profit margin of 4.9 percent, 1.5 percentage points higher than that of the Top 250. Twentynine Fastest 50 retailers were also among the 50 fastest-growing retailers in 2013. 


\begin{tabular}{|c|c|c|c|c|c|c|c|c|}
\hline $\begin{array}{l}\text { Growth } \\
\text { rank }\end{array}$ & $\begin{array}{l}\text { Top } \\
250 \\
\text { rank }\end{array}$ & Name of company & $\begin{array}{l}\text { Country } \\
\text { of origin }\end{array}$ & $\begin{array}{r}2013 \\
\text { retail } \\
\text { revenue } \\
\text { (US \$mil) }\end{array}$ & Dominant operational format & $\begin{array}{r}2008-2013 \\
\text { retail } \\
\text { revenue } \\
\text { CAGR }^{1}\end{array}$ & $\begin{array}{r}2013 \\
\text { retail } \\
\text { revenue } \\
\text { growth }\end{array}$ & $\begin{array}{r}2013 \\
\text { net } \\
\text { profit } \\
\text { margin }\end{array}$ \\
\hline 1 & 92 & JD.com, Inc. & China & 10,827 & Non-Store & $123.6 \%$ & $66.2 \%$ & $-0.1 \%$ \\
\hline 2 & 189 & $\begin{array}{l}\text { Yonghui Superstores } \\
\text { Co., Ltd. }\end{array}$ & China & 4,733 & Hypermarket/Supercenter/Superstore & $38.8 \%$ & $23.4 \%$ & $2.4 \%$ \\
\hline 3 & 43 & Albertson's LLC & U.S. & $23,000^{\mathrm{e}}$ & Supermarket & $38.6 \%$ & $489.7 \%$ & $\mathrm{n} / \mathrm{a}$ \\
\hline 4 & 111 & Jumbo Groep Holding B.V. & Netherlands & $9,420^{9}$ & Supermarket & $36.9 \%$ & $1.9 \%$ & $\mathrm{n} / \mathrm{a}$ \\
\hline 5 & 188 & $\begin{array}{l}\text { Chongqing Department } \\
\text { Store Co., Ltd. }\end{array}$ & China & 4,733 & Department Store & $36.0 \%$ & $7.1 \%$ & $2.6 \%$ \\
\hline 6 & 104 & $\begin{array}{l}\text { Chow Tai Fook Jewellery } \\
\text { Group Limited }\end{array}$ & $\begin{array}{l}\text { Hong Kong } \\
\text { SAR }\end{array}$ & $9,979^{* *}$ & Other Specialty & $33.3 \%$ & $34.8 \%$ & $9.6 \%$ \\
\hline 7 & 102 & $\begin{array}{l}\text { Southeastern Grocers, } \\
\text { LLC }\end{array}$ & U.S. & $10,126^{e}$ & Supermarket & $32.2 \%$ & $19.2 \%$ & $\mathrm{n} / \mathrm{a}$ \\
\hline 8 & 128 & $\begin{array}{l}\text { Steinhoff International } \\
\text { Holdings Ltd. }\end{array}$ & S. Africa & 8,217 & Other Specialty & $31.5 \%$ & $21.6 \%$ & $8.4 \%$ \\
\hline 9 & 166 & OJSC Dixy Group & Russia & 5,628 & Supermarket & $30.2 \%$ & $22.8 \%$ & $1.7 \%$ \\
\hline 10 & 241 & SMU S.A. & Chile & $3,823^{* *}$ & Supermarket & $29.8 \%$ & $-9.2 \%$ & $-27.8 \%$ \\
\hline 11 & 225 & PRADA Group & Italy & 3,989 & Apparel/Footwear Specialty & $28.0 \%$ & $12.5 \%$ & $17.8 \%$ \\
\hline 12 & 55 & OJSC "Magnit" & Russia & 18,197 & Convenience/Forecourt Store & $27.9 \%$ & $26.2 \%$ & $6.1 \%$ \\
\hline 13 & 247 & Magazine Luiza SA & Brazil & 3,758 & Electronics Specialty & $27.5 \%$ & $14.5 \%$ & $1.3 \%$ \\
\hline 14 & 15 & Amazon.com, Inc. & U.S. & 60,903 & Non-Store & $26.7 \%$ & $17.7 \%$ & $0.4 \%$ \\
\hline 15 & 190 & $\begin{array}{l}\text { Ascena Retail Group, } \\
\text { Inc. }\end{array}$ & U.S. & 4,715 & Apparel/Footwear Specialty & $26.7 \%$ & $40.6 \%$ & $3.2 \%$ \\
\hline 16 & 196 & Lenta Group & Russia & 4,539 & Hypermarket/Supercenter/Superstore & $23.2 \%$ & $31.3 \%$ & $4.9 \%$ \\
\hline 17 & 151 & $\begin{array}{l}\text { BiM Birleşik } \\
\text { Mağazalar A.Ş. }\end{array}$ & Turkey & 6,242 & Discount Store & $22.8 \%$ & $19.6 \%$ & $3.5 \%$ \\
\hline 18 & 46 & $\begin{array}{l}\text { Apple Inc./Apple Retail } \\
\text { Stores }\end{array}$ & U.S. & 20,228 & Electronics Specialty & $22.6 \%$ & $7.4 \%$ & $21.7 \%$ \\
\hline 19 & 78 & $\begin{array}{l}\text { China Resources } \\
\text { Enterprise, Limited }\end{array}$ & $\begin{array}{l}\text { Hong Kong } \\
\text { SAR }\end{array}$ & 12,258 & Hypermarket/Supercenter/Superstore & $22.4 \%$ & $14.0 \%$ & $2.2 \%$ \\
\hline 20 & 206 & O'Key Group S.A. & Russia & 4,330 & Hypermarket/Supercenter/Superstore & $22.3 \%$ & $18.8 \%$ & $3.6 \%$ \\
\hline 21 & 132 & $\begin{array}{l}\text { Compagnie Financière } \\
\text { Richemont SA }\end{array}$ & Switzerland & 7,841 & Other Specialty & $20.5 \%$ & $7.5 \%$ & $19.4 \%$ \\
\hline 22 & 170 & Nike, Inc. & U.S. & 5,304 & Apparel/Footwear Specialty & $19.5 \%$ & $22.6 \%$ & $9.7 \%$ \\
\hline 23 & 248 & HTM-Group & France & $3,706^{e}$ & Electronics Specialty & $19.1 \%$ & $1.5 \%$ & $\mathrm{n} / \mathrm{a}$ \\
\hline 24 & 183 & $\begin{array}{l}\text { Emke Group/Lulu Group } \\
\text { International }\end{array}$ & UAE & $5,000^{\mathrm{e}}$ & Hypermarket/Supercenter/Superstore & $18.7 \%$ & $9.6 \%$ & $\mathrm{n} / \mathrm{a}$ \\
\hline 25 & 224 & Forever 21, Inc. & U.S. & $4,000^{\mathrm{e}}$ & Apparel/Footwear Specialty & $18.7 \%$ & $8.1 \%$ & $\mathrm{n} / \mathrm{a}$ \\
\hline 26 & 40 & Lotte Shopping Co., Ltd. & S. Korea & 24,601 & Hypermarket/Supercenter/Superstore & $17.5 \%$ & $13.4 \%$ & $3.1 \%$ \\
\hline 27 & 113 & $\begin{array}{l}\text { CP ALL Public Company } \\
\text { Limited }\end{array}$ & Thailand & 9,286 & Convenience/Forecourt Store & $17.5 \%$ & $46.0 \%$ & $3.7 \%$ \\
\hline 28 & 144 & $\begin{array}{l}\text { Associated British Foods } \\
\text { plc/Primark }\end{array}$ & U.K. & 6,673 & Apparel/Footwear Specialty & $17.2 \%$ & $22.0 \%$ & $4.8 \%$ \\
\hline 29 & 153 & $\begin{array}{l}\text { Central Retail } \\
\text { Corporation Ltd. }\end{array}$ & Thailand & $6,207^{e}$ & Department Store & $17.2 \%$ & $25.8 \%$ & $\mathrm{n} / \mathrm{a}$ \\
\hline 30 & 149 & Coppel S.A. de C.V. & Mexico & 6,304 & Department Store & $17.1 \%$ & $17.0 \%$ & $13.5 \%$ \\
\hline 31 & 192 & $\begin{array}{l}\text { OJSC "Company } \\
\text { M.video" }\end{array}$ & Russia & 4,657 & Electronics Specialty & $16.8 \%$ & $10.8 \%$ & $3.9 \%$ \\
\hline 32 & 59 & $\begin{array}{l}\text { Suning Commerce Group } \\
\text { Co., Ltd. }\end{array}$ & China & 16,616 & Electronics Specialty & $16.3 \%$ & $4.6 \%$ & $0.4 \%$ \\
\hline 33 & 134 & $\begin{array}{l}\text { FEMSA Comercio, S.A. } \\
\text { de C.V. }\end{array}$ & Mexico & 7,655 & Convenience/Forecourt Store & $15.7 \%$ & $12.9 \%$ & $\mathrm{n} / \mathrm{a}$ \\
\hline
\end{tabular}

Companies in bold italic type were also among the 50 fastest-growing retailers in 2013.

Fastest 50 and Top 250 composite net profit margins exclude results for companies that are not primarily retailers.

${ }^{1}$ Compound annual growth rate

** Revenue includes wholesale and retail sales

$\mathrm{e}=$ estimate

$\mathrm{g}=$ gross turnover as reported by company

Source: Published company data and Planet Retail 


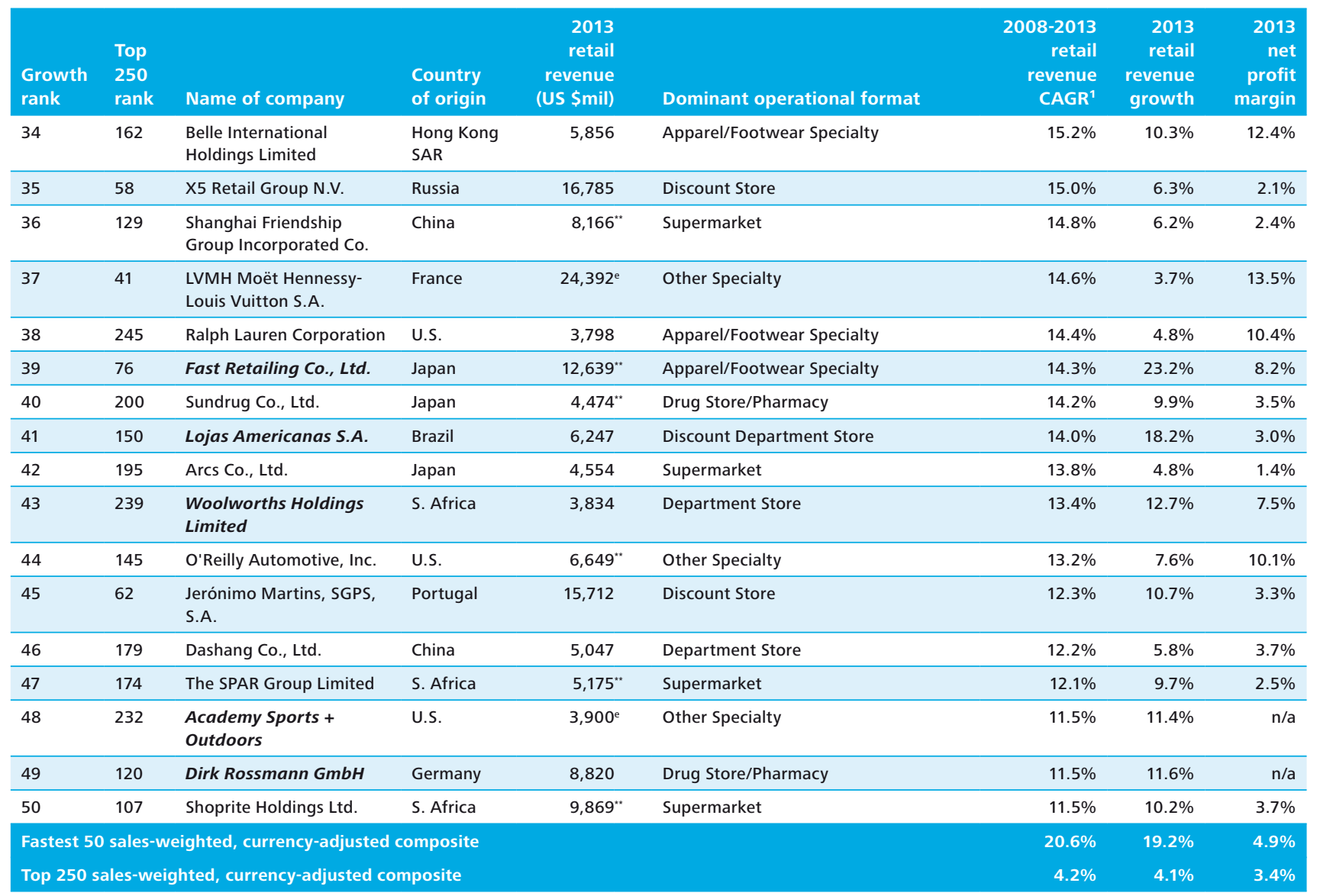

Companies in bold italic type were also among the 50 fastest-growing retailers in 2013.

Fastest 50 and Top 250 composite net profit margins exclude results for companies that are not primarily retailers.

${ }^{1}$ Compound annual growth rate

** Revenue includes wholesale and retail sales

e = estimate

$\mathrm{g}=$ gross turnover as reported by company

Source: Published company data and Planet Retail 


\section{Top 50 e-retailers}

E-retailing, as defined in this analysis, includes B2C e-commerce only, where the business owns the inventory and sales are made directly to the consumer. Companies that primarily operate as e-marketplaces, or facilitators, that aggregate many sellers are excluded from the Top 50 e-retailer analysis as their revenues are largely derived from fees and commissions on sales from third-parties - consumers or other businesses that own the inventory - rather than directly from the sale of goods.

Retailers extend online presence with multiple e-commerce models

Global Internet penetration continues to rise - particularly in Africa, the Middle East, Southeast Asia, and Central Asia. Along with a greater number of Internet users is a dramatic increase in the number of mobile internet-enabled devices, such as smartphones and tablets. The embrace of these technologies is having a dramatic impact on media usage, marketing, and e-commerce, as well as enabling new consumer shopping behaviors.

As e-commerce competition heats up, retailers pursuing greater online sales face an increasingly crowded, complex, and fragmented market. In order to grab consumers' attention and extend their online presence, many sellers are utilizing multiple online models including:

- Directly operated online storefront

- Online storefront on third-party marketplace

- Incorporating third-party marketplace into retailer's own online storefront

- Expansion of click and collect services

- Selling products to other e-retailers on a wholesale or consignment basis

- Social networking and commerce applications

- Affiliate marketing programs, where one business sells the products of another in return for a commission

- Paid search and comparison shopping sites as key influencers of consumer purchase decisions

As online sales accelerate, especially in developing markets, retailers are increasingly viewing e-commerce as a key element of their global expansion strategies. The opportunity to engage in international e-commerce is helping drive the growth of e-marketplaces and alternative online channels. This rapidly evolving e-commerce world is evident in the composition of e-50, the world's 50 largest e-retailers, which collectively employ all of the e-retailing channels and online consumer touch points outlined above.
Amazon continues to dominate world of e-retailing Analysis of the e-50 retailers shows:

- Amazon continued to dominate the world of e-retailing with 2013 net product sales (i.e., sales where Amazon is the seller of record) of nearly US\$61 billion. JD.com, formerly known as Beijing Jingdong Century Trade Co., Ltd. and 360buy Jingdong Inc., ran a distant second with e-retail sales of US\$10.8 billion, followed by Wal-Mart with approximately US\$10 billion in e-commerce sales.

- The Top 250 Global Powers of Retailing accounted for nearly three-quarters of the e-50 in 2013 (37 companies). Although the world's 250 largest retailers continued to dominate the list, they accounted for two fewer top 50 e-retailers compared with 2012.

- The majority of the e-50 (39 companies) are multi-channel retailers with bricks-and-mortar stores as well as online or other non-store operations. However this is down from 42 such companies in 2012. While many pure-play e-retailers are growing rapidly, with more likely to join the e-50 in the near future, only two of the 11 non-store or web-only e-50 retailers in 2013 were large enough to rank among the Top 250 - Amazon.com and JD.com.

- Half of the e-50 are based in the United States (down from 28 in 2012), followed by Europe (19 companies, up two from 2012) - especially the U.K. (10) and France (5); only five are emerging market companies (4 China, 1 Brazil). Online marketplaces rather than e-retailers tend to serve as the primary e-commerce model in Asia and Latin America (see "The growing power of the e-marketplace" below).

- E-50 retailers grew their digital sales at a rapid pace in 2013, averaging 26.6 percent.

- Among the e-50, e-commerce still accounts for a small share of mass merchants' and food retailers' revenue - typically just 2-4 percent. For department stores and specialty apparel retailers, online sales accounted for a larger 8-16 percent share, and reached $20+$ percent for several consumer electronics retailers. 


\begin{tabular}{|c|c|c|c|c|c|c|c|}
\hline $\begin{array}{l}\text { Top } \\
\text { e-retailer } \\
\text { sales rank } \\
\text { FY13 }\end{array}$ & $\begin{array}{l}\text { Top } \\
250 \\
\text { sales } \\
\text { rank } \\
\text { FY13 }\end{array}$ & Name of company & $\begin{array}{l}\text { Country } \\
\text { of origin }\end{array}$ & $\begin{array}{r}\text { FY13 } \\
\text { e-commerce } \\
\text { retail sales } \\
\text { (US\$ mil) }\end{array}$ & $\begin{array}{r}\text { FY13 } \\
\text { e-commerce } \\
\text { retail sales } \\
\% \text { of total } \\
\text { retail revenue }\end{array}$ & $\begin{array}{r}\text { FY13 } \\
\text { e-commerce } \\
\text { growth rate }\end{array}$ & Comments \\
\hline 1 & 15 & Amazon.com Inc. & U.S. & $60,903.0$ & $100.0 \%$ & $17.7 \%$ & Online direct sales; excludes third party sales \\
\hline 2 & 92 & JD.com, Inc. & China & $10,826.8$ & $100.0 \%$ & $66.2 \%$ & $\begin{array}{l}\text { Online direct sales; formerly known as Beijing } \\
\text { Jingdong Century Trade Co., Ltd. and 360buy } \\
\text { Jingdong Inc. }\end{array}$ \\
\hline 3 & 1 & Wal-Mart Stores, Inc. & U.S. & $10,000.0^{\mathrm{e}}$ & $2.1 \%$ & $29.9 \%$ & \\
\hline 4 & 46 & Apple Inc. & U.S. & $9,000.0^{\mathrm{e}}$ & $30.8 \%$ & $\mathrm{n} / \mathrm{a}$ & $\begin{array}{l}\text { Estimated sales of Store.Apple.com; excludes } \\
\text { iTunes store, App store, iBooks store }\end{array}$ \\
\hline 5 & 70 & $\begin{array}{l}\text { Otto (GmbH \& } \\
\text { Co KG) }\end{array}$ & Germany & $8,188.9^{e}$ & $61.3 \%$ & $7.0 \%$ & \\
\hline 6 & 5 & Tesco PLC & U.K. & $5,250.5^{e}$ & $5.3 \%$ & $11.0 \%$ & \\
\hline 7 & 99 & $\begin{array}{l}\text { Liberty Interactive } \\
\text { Corporation }\end{array}$ & U.S. & $4,884.0$ & $47.4 \%$ & $10.9 \%$ & $\begin{array}{l}\text { Includes QVC.com plus company's other e-retail } \\
\text { subsidiaries }\end{array}$ \\
\hline 8 & 13 & $\begin{array}{l}\text { Casino Guichard- } \\
\text { Perrachon S.A. }\end{array}$ & France & $3,952.8$ & $6.2 \%$ & $19.4 \%$ & $\begin{array}{l}\text { Includes e-commerce sales for Cdiscount, Nova } \\
\text { Pontocom, and exito.com }\end{array}$ \\
\hline 9 & 59 & $\begin{array}{l}\text { Suning Commerce } \\
\text { Group Co., Ltd. }\end{array}$ & China & $3,536.3$ & $21.3 \%$ & $43.9 \%$ & \\
\hline 10 & 34 & Macy's, Inc. & U.S. & $3,100.0^{\mathrm{e}}$ & $11.1 \%$ & $37.8 \%$ & \\
\hline 11 & 2 & $\begin{array}{l}\text { Costco Wholesale } \\
\text { Corporation }\end{array}$ & U.S. & $3,086.1^{e}$ & $2.9 \%$ & $47.0 \%$ & \\
\hline 12 & 25 & Best Buy Co., Inc. & U.S. & $3,044.0^{\mathrm{e}}$ & $7.2 \%$ & $19.8 \%$ & Estimate for domestic online sales only \\
\hline 13 & 117 & $\begin{array}{l}\text { Home Retail } \\
\text { Group plc }\end{array}$ & U.K. & $2,906.8^{e}$ & $32.6 \%$ & $7.0 \%$ & \\
\hline 14 & 150 & $\begin{array}{l}\text { Lojas Americanas } \\
\text { S.A. }\end{array}$ & Brazil & $2,838.1$ & $45.4 \%$ & $26.5 \%$ & $\begin{array}{l}\text { B2W - Companhia Digital portfolio } \\
\text { comprises retailer brands Americanas. } \\
\text { com, Submarino, Shoptime, and SouBarato } \\
\text { plus Ingresso.com (ticket sales), Submarino } \\
\text { Finance (credit card), and B2W Viagens (travel } \\
\text { agency), which operate through the Internet, } \\
\text { telesales, catalogs, TV and kiosks. }\end{array}$ \\
\hline 15 & 9 & $\begin{array}{l}\text { The Home Depot, } \\
\text { Inc. }\end{array}$ & U.S. & $2,750.0^{\mathrm{e}}$ & $3.5 \%$ & $52.8 \%$ & \\
\hline 16 & $\mathrm{n} / \mathrm{a}$ & Newegg Inc. & U.S. & $2,700.0^{\mathrm{e}}$ & $100.0 \%$ & $-3.6 \%$ & $\begin{array}{l}\text { Includes Newegg Marketplace revenue that } \\
\text { could not be broken out }\end{array}$ \\
\hline 17 & 81 & Staples, Inc. & U.S. & $2,500.0^{e}$ & $20.6 \%$ & $0.0 \%$ & $\begin{array}{l}\text { Estimated B2C e-commerce sales; excludes B2B } \\
\text { e-commerce sales }\end{array}$ \\
\hline 18 & $\mathrm{n} / \mathrm{a}$ & Zalando AG & Germany & $2,340.4$ & $100.0 \%$ & $52.1 \%$ & \\
\hline 19 & $\mathrm{n} / \mathrm{a}$ & Shop Direct Group & U.K. & $2,322.6^{e}$ & $84.0 \%$ & $8.3 \%$ & \\
\hline 20 & 61 & The Gap, Inc. & U.S. & $2,260.0$ & $14.0 \%$ & $21.5 \%$ & \\
\hline 21 & 68 & $\begin{array}{l}\text { John Lewis } \\
\text { Partnership plc }\end{array}$ & U.K. & $2,204.3^{9}$ & $15.6 \%$ & $22.8 \%$ & \\
\hline 22 & $\mathrm{n} / \mathrm{a}$ & Vente.privee.com & France & $2,125.2$ & $100.0 \%$ & $23.0 \%$ & \\
\hline 23 & 163 & Next plc & U.K. & $2,103.9^{\text {NS }}$ & $36.0 \%$ & $12.4 \%$ & Includes catalog sales \\
\hline 24 & 23 & $\begin{array}{l}\text { Centres Distributeurs } \\
\text { E. Leclerc }\end{array}$ & France & $1,952.5^{9}$ & $4.1 \%$ & $63.0 \%$ & Gross sales through E.Leclerc Drive \\
\hline 25 & 204 & $\begin{array}{l}\text { Williams-Sonoma, } \\
\text { Inc. }\end{array}$ & U.S. & $1,950.0$ & $44.4 \%$ & $18.2 \%$ & \\
\hline 26 & 93 & L Brands, Inc. & U.S. & $1,766.0^{\mathrm{NS}}$ & $16.4 \%$ & $-2.4 \%$ & Includes catalog sales \\
\hline 27 & 52 & Kohl's Corp. & U.S. & $1,700.0$ & $8.9 \%$ & $20.4 \%$ & \\
\hline 28 & 10 & Target Corp. & U.S. & $1,700.0^{e}$ & $2.3 \%$ & $\mathrm{n} / \mathrm{a}$ & \\
\hline 29 & $\mathrm{n} / \mathrm{a}$ & $\begin{array}{l}\text { Vipshop Holdings } \\
\text { Limited }\end{array}$ & China & $1,680.6$ & $100.0 \%$ & $143.5 \%$ & \\
\hline 30 & 7 & Metro Ag & Germany & $1,657.7$ & $1.9 \%$ & $54.5 \%$ & \\
\hline
\end{tabular}




\begin{tabular}{|c|c|c|c|c|c|c|c|}
\hline $\begin{array}{l}\text { Top } \\
\text { e-retailer } \\
\text { sales rank } \\
\text { FY13 }\end{array}$ & $\begin{array}{l}\text { Top } \\
250 \\
\text { sales } \\
\text { rank } \\
\text { FY13 }\end{array}$ & Name of company & $\begin{array}{l}\text { Country } \\
\text { of origin }\end{array}$ & $\begin{array}{r}\text { FY13 } \\
\text { e-commerce } \\
\text { retail sales } \\
\text { (US\$ mil) }\end{array}$ & $\begin{array}{r}\text { FY13 } \\
\text { e-commerce } \\
\text { retail sales } \\
\% \text { of total } \\
\text { retail revenue }\end{array}$ & $\begin{array}{r}\text { FY13 } \\
\text { e-commerce } \\
\text { growth rate }\end{array}$ & Comments \\
\hline 31 & 80 & Nordstrom, Inc. & U.S. & $1,622.0$ & $13.3 \%$ & $27.8 \%$ & \\
\hline 32 & 85 & Dixons Retail plc & U.K. & $1,601.6$ & $13.9 \%$ & $16.0 \%$ & \\
\hline 33 & 26 & J Sainsbury plc & U.K. & $1,589.9^{e}$ & $4.2 \%$ & $12.4 \%$ & \\
\hline 35 & $\mathrm{n} / \mathrm{a}$ & $\begin{array}{l}\text { Tencent Holdings } \\
\text { Limited }\end{array}$ & China & $1,582.5$ & $100.0 \%$ & $121.2 \%$ & $\begin{array}{l}\text { Mostly 51Buy.com (Yixun in Chinese). Includes } \\
\text { some marketplace revenue that could not be } \\
\text { broken out. }\end{array}$ \\
\hline 36 & 14 & Groupe Auchan SA & France & $1,507.6$ & $2.4 \%$ & $11.9 \%$ & \\
\hline 37 & 24 & $\begin{array}{l}\text { Koninklijke Ahold } \\
\text { N.V. }\end{array}$ & Netherlands & $1,442.5$ & $3.3 \%$ & $30.8 \%$ & \\
\hline 41 & 60 & $\begin{array}{l}\text { Marks \& Spencer } \\
\text { Group plc }\end{array}$ & U.K. & $1,272.0$ & $7.8 \%$ & $22.8 \%$ & \\
\hline 42 & $\mathrm{n} / \mathrm{a}$ & Ocado Group plc & U.K. & $1,239.5$ & $100.0 \%$ & $16.7 \%$ & \\
\hline 43 & $\mathrm{n} / \mathrm{a}$ & L.L. Bean, Inc. & U.S. & $1,200.0^{\mathrm{e}}$ & $76.9 \%$ & $5.3 \%$ & \\
\hline 44 & 29 & Sears Holdings Corp. & U.S. & $1,200.0^{\mathrm{e}}$ & $3.3 \%$ & $\mathrm{n} / \mathrm{a}$ & Estimate excludes sales on Sears Marketplace \\
\hline 45 & $\mathrm{n} / \mathrm{a}$ & Systemax Inc. & U.S. & $1,193.9^{\mathrm{NS}}$ & $100.0 \%$ & $-18.2 \%$ & Includes catalog sales \\
\hline 46 & $\mathrm{n} / \mathrm{a}$ & ASOS Plc & U.K. & $1,178.7$ & $100.0 \%$ & $40.0 \%$ & $\begin{array}{l}\text { Online direct sales; excludes third party } \\
\text { revenues and delivery receipts }\end{array}$ \\
\hline 47 & 77 & Toys "R" Us, Inc. & U.S. & $1,157.0^{\mathrm{e}}$ & $9.2 \%$ & $2.5 \%$ & \\
\hline 48 & 18 & Woolworths Limited & Australia & $1,102.0^{\mathrm{e}}$ & $2.0 \%$ & $50.0 \%$ & \\
\hline
\end{tabular}

e = estimated $\quad \mathrm{g}=$ gross transaction volume $\quad \mathrm{NS}=$ total non-store sales $\quad \mathrm{n} / \mathrm{a}=$ not among the Top 250 in 2013




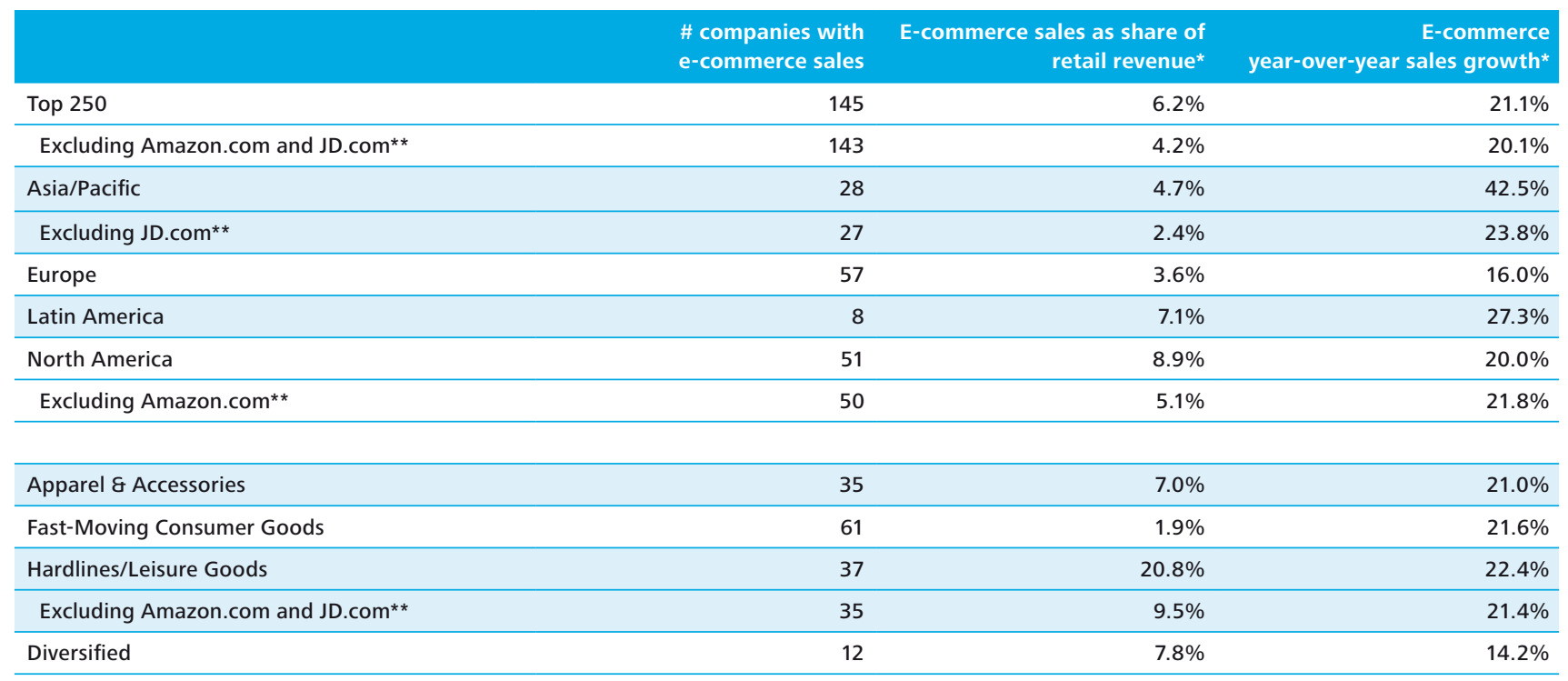

Africa/Middle East region excluded as e-commerce sales available for only one retailer

* Sales-weighted composites

** Amazon.com and JD.com are the only two online-only retailers in the Top 250

In addition to identifying the world's 50 largest e-retailers, e-commerce activity for all Top 250 retailers was analyzed. For 2013, information was available for 185 companies (either as reported by the company or estimated by Planet Retail or Internet Retailer). Of these 185 companies:

- About one-fifth (40) did not have a transactional e-commerce website in 2013. This is down from more than one-quarter in 2012 as more retailers got in the game. Most of the companies without e-commerce were supermarket operators and hard discount retailers.

- The 145 Top 250 companies with e-commerce operations generated 6.2 percent of their combined retail revenue online in 2013. Without Amazon.com and JD.com - the two online-only retailers among the Top 250 - e-commerce as a share of total retail revenue falls by one-third to 4.2 percent. On a geographic basis, e-commerce accounted for the largest share of retail revenue for the North American retailers - 8.9 percent. However, if Amazon is factored out of the North American calculation, online sales as a share of total sales was highest for the Latin American retailers at 7.1 percent. E-commerce produced the smallest share of sales, 3.6 percent, for the European retailers a reflection of the relatively low level of online sales penetration among some of the region's biggest companies, many of which are primarily food retailers. E-commerce accounted for 4.7 percent of sales for the Asia/Pacific retailers. Excluding JD.com, this region's online share drops to just 2.4 percent.
- From a product perspective, hardlines and leisure goods retailers derived a larger share of revenue from e-commerce operations than did the other sectors - 20.8 percent on a composite basis. Excluding Amazon.com and JD.com, this sector still had the highest e-commerce penetration, although it drops by more than half. Not surprisingly, e-commerce penetration was lowest among retailers of fast-moving consumer goods at just 1.9 percent of sales.

- Online sales grew at a composite rate of 21.1 percent for the 145 Top 250 retailers with e-commerce operations. From a regional perspective, e-commerce sales growth ranged from 16.0 percent for the European retailers to 42.5 percent for the Asia/Pacific retailers. However, Asia/Pacific drops to 23.8 percent without the region's largest e-commerce retailer JD.com, which grew its direct sales 66.2 percent in 2013. If JD.com is excluded from the Asia/ Pacific result, the Latin American retailers have the highest online sales growth -27.3 percent. On a product basis, composite e-commerce growth was fairly consistent across the three primary product sectors, ranging between 21 and 22.4 percent. The diversified group reported the lowest composite growth rate in e-commerce sales. 
Top e-marketplace sites, 2013

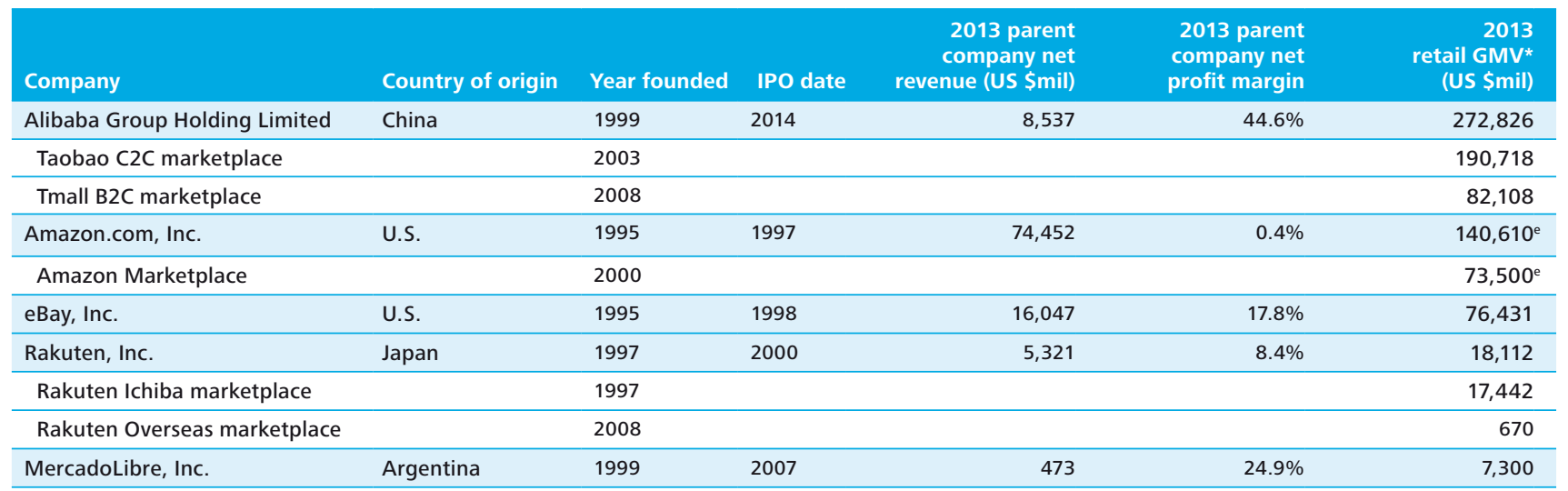

* Gross Merchandise Value, or the value of all merchandise sold

The growing power of the e-marketplace

As many globally minded retailers have already learned on the ground, competing against well-entrenched domestic players is difficult. To gain acceptance with foreign consumers online is no different than through bricks and mortar expansion - you need to localize your offer and your operations.

Capitalizing on that reality, third-party marketplaces have become an increasingly important driver of growth for online retailers. This is especially evident in developing markets where consumers often navigate directly to these marketplaces, many of which have become well-recognized and trusted brands. Their selection and convenience provide value for consumers, while their scale and reach provide merchants access to a large base of potential customers.

In addition to cost-effective customer acquisition in often unfamiliar territory, the marketplace infrastructure offers sellers a range of essential support services--including payment, fulfillment, customer service, marketing, and promotion - necessary to operate their businesses. Without the capital investment, time, and risk required to launch their own country- or region-specific e-commerce operations, many retailers are finding that e-marketplaces can be a quicker, easier, and more efficient way to tap into growth markets around the world.

Alibaba Group, China's most-popular e-commerce destination, is the world's largest e-marketplace company with gross merchandise value (GMV) - the value of all merchandise sold - of US\$272.8 billion in 2013, nearly twice that of Amazon. Alibaba operates exclusively a third-party platform business model consisting of two main e-commerce sites: Taobao, where consumers sell to other consumers, and Tmall, where retailers sell directly to consumers.

Amazon, the world's largest e-retailer, offers products from its own inventory as well as products sold by others on Amazon Marketplace. The company's total GMV in 2013 is estimated at more than US\$140 billion, with US\$73.5 billion generated by third-party sellers.
Amazon continues to disrupt markets whenever it enters a new category. The company is expanding AmazonFresh, an online grocery service it first began testing in Seattle in 2007. Amazon Fashion is another emerging business. While Amazon has sold apparel, shoes and accessories for years, the company has intensified its focus on the fashion category in the past few years, targeting contemporary, high-end brands.

eBay ranks as the third-largest e-marketplace with total GMV of US\$76.4 billion. While it offers only third-party products, the company's reported GMV does not distinguish its B2C business from its $\mathrm{C} 2 \mathrm{C}$ business.

Japan's Rakuten operates the world's fourth-largest e-marketplace. Total GMV of US\$18.1 billion is derived primarily from its domestic online shopping mall, Rakuten Ichiba. The company launched its first e-commerce operation overseas in Taiwan in 2008. In 2010, Rakuten acquired Buy.com, one of the leading U.S. e-marketplace companies (now Rakuten.com).

MercadoLibre (literally "free market" in Spanish) hosts the largest online commerce platform in Latin America. It began operations in Argentina in 1999 and rapidly expanded to Brazil, other South American countries, and Mexico. In 2001, MercadoLibre entered into a strategic alliance with eBay, gaining proprietary market information and acquiring eBay's Brazilian unit, which helped to consolidate the company's leadership position in the region. The strategic alliance ended in 2006, just prior to MercadoLibre's IPO in 2007. The company's GMV in 2013 was US\$7.3 billion.

In addition, a growing list of retailers including Sears, Wal-Mart, Best Buy, and Tesco are incorporating third-party marketplaces into their own online storefronts. This provides their customers with much greater choice without having to look elsewhere and allows the retailer to capture a greater share of e-commerce revenue. 


\section{Q ratio analysis for Global Powers}

Over the last ten years, this report has offered an analysis of the $\mathrm{Q}$ ratios of publicly traded retailers from our Top 250 list. Before explaining the inferences to be drawn from this analysis, let us consider the meaning and importance of the $\mathrm{Q}$ ratio.

What is the $\mathbf{Q}$ ratio, and why is it important?

In today's world, the global business environment is characterized by intense competition combined with downward pressure on retail prices, slow growth in major developed markets such as Europe, slower growth in emerging markets than in recent years, volatile input prices combined with consumer resistance to higher retail prices, excess store capacity in many developed markets, and a continued shift toward online retailing in which consumers often perceive everything to be a commodity. What this means is that, in order for retailers to prosper, they must distinguish themselves from competitors in order to have pricing power that leads to higher margins. This means having strong brand identity, offering consumers a superior shopping experience, and being clearly differentiated from competitors. The latter can entail unique merchandise offerings including private brands, unique store formats and designs, and superior customer experience. If a publicly traded retailer has these characteristics, the financial markets are likely to reward such a retailer. That is where the $\mathrm{Q}$ ratio comes in.

The $\mathrm{Q}$ ratio is the ratio of a publicly traded company's market capitalization to the value of its tangible assets. If this ratio is greater than one, it means that financial market participants believe that part of a company's value comes from its non-tangible assets. These can include such things as brand equity, differentiation, innovation, customer experience, market dominance, customer loyalty, and skillful execution. The higher the Q ratio, the greater share of a company's value that stems from such non-tangibles. $A \mathrm{Q}$ ratio of less than one, on the other hand, indicates failure to generate value on the basis of non-tangible assets. It indicates that the financial markets view a retailer's strategy as unable to generate a sufficient return on physical assets. Indeed it suggests an arbitrage opportunity. That is, if a company's $Q$ ratio is less than one, theoretically a company could be purchased through equity markets and the tangible assets could then be sold at a profit.

In our analysis, we have calculated the Q ratio for all 156 publicly traded retailers on our top 250 list. The calculation is based on companies' assets at the end of the latest fiscal year as well as the market capitalization as calculated in late October 2014.

\section{Which companies have notable Q ratios?}

Once again, the top spot on our Q ratio list goes to H\&M, the legendary Swedish apparel retailer. H\&M has been at or near the top of this list since we started compiling this information. It is clear that H\&M has been successful in differentiating from competitors and building strong brand equity around the world. Thus, its position on the list is no surprise. This, however, is not a skill possessed by H\&M alone. Indeed seven of the top ten retailers on our Q ratio list are in the apparel/footwear specialty category. Four are European, two are from the U.S., and one is from Japan. In addition, the top ten includes two discount stores. Plus, two of the seven apparel/footwear specialty retailers (TJX and Ross) are principally discount oriented. Thus, four of the top ten are focused on offering consumers low prices.

Also, of the top 20 retailers on our Q ratio list, eleven are in the United States and three are based in emerging markets, down from four last year. On the other hand, of the bottom 20 retailers on our list, nine are based in Japan, down from 11 last year. Evidently, U.S.-based retailers account for a disproportionate share of those that have generated considerable value through their non-tangible assets. Japanese retailers disproportionately represent those that have not - although the situation for Japanese retailers has evidently improved - possibly due to greater optimism about the future of the Japanese economy.

\section{Highlights}

This year we analyzed the financial results of 156 publicly traded companies on our list of the top 250 retailers in the world. This is down from 159 companies analyzed last year. The composite Q ratio for all companies was 1.130, down from 1.297 last year. Yet this year's composite Q remains well below the 1.57 recorded in 2008 just before the start of the global financial crisis. Of the 156 companies on the list, 73 have Q ratios above one and 83 have $\mathrm{Q}$ ratios below one. We also examined the composite $\mathrm{Q}$ ratio by country, region, retail format, and dominant product category. We only calculated a composite ratio when there were three or more companies in a particular category. 
The retail formats with the highest composite Q ratios are electronics, apparel/footwear, home improvement, and other specialty. The electronics specialty category is dominated by Apple, Inc. which accounts for more than 94 percent of the market capitalization of the electronics specialty companies on our list. If Apple is excluded from the list, the composite $Q$ ratio for the electronics category drops from 2.392 to 0.512 . This is not entirely surprising. Most electronics retailers now face considerable competition from online sellers, online sites of manufacturers, and discounters. It is difficult to differentiate when selling products that are often perceived as commodities. Apparel retailers have become extremely important global players, with a combined market capitalization more than three times higher than the department store industry. The Q ratio of apparel retailers (1.727) is nearly three times higher than that of the department store industry (0.599). Yet this is down from last year when apparel retailers beat department store retailers by nearly four to one. The lowest composite $\mathrm{Q}$ ratio belongs to the electronics industry less Apple. After that, the lowest ratios are for convenience retailers and hypermarkets. The latter is an industry that has faced considerable competitive challenges in recent years. Moreover, it is a format where clear differentiation is difficult and where price competition is brutal. Hence, the low Q ratio is not entirely surprising. Notably, the composite Q ratio for discounters is considerably higher than that of hypermarkets. This is not surprising given that two discounters are among the top ten retailers ranked by $\mathrm{Q}$ ratio.

Of the four merchandise categories, the two with the highest composite Q ratios are hardlines/leisure (2.085) and apparel and accessories (1.230). Yet given the dominance of Apple in the hardlines category (accounting for more than half the market capitalization of the category), it is notable that the apparel and accessories category has the highest composite Q ratio when Apple is excluded from this analysis. As usual, the category of diversified retailers has the lowest composite $\mathrm{Q}$ ratio. Of the 15 retailers in the category, only three have $\mathrm{Q}$ ratios above one. Retailers specializing in fast moving consumer goods (FMCG), once again, have a composite $\mathrm{Q}$ ratio lower than one.
We also analyzed the composite Q ratios of countries, provided that there are three or more companies from a given country. We excluded countries with only one or two retailers from this analysis. The weakest composite $\mathrm{Q}$ ratios are those of China, Germany, and South Korea in that order. The highest composite $\mathrm{Q}$ ratios are found in Sweden, the U.S., and Russia. By region, there is a stark divide between North America (1.760) and the Africa/ Middle East region (1.315) and every other region (ranging from 0.469 for Asia Pacific to 0.847 for Europe). Moreover, the higher $\mathrm{Q}$ ratio for North America is due to the higher Q ratio for the U.S. The other North American country, Canada, has a relatively low $\mathrm{Q}$ ratio. There are many possible explanations for the relatively high Q ratio for the United States. Some observers will say that it reflects strong brand equity, clear differentiation, and a successful transition to online retailing in the United States. Others will note that these trends are often absent in other regions. Still others, however, might argue that the higher ratio for the U.S. simply reflects the inflated values of U.S. equities in general, itself the result of aggressive U.S. monetary policy. Of course Japan has had an aggressive monetary policy and its equity market has soared. Still, the composite Q ratio for Japan is relatively low. As for Europe, its economy has been weak and this has been reflected in poorer performance of its equity market in general.

\section{Top retailers by $\mathrm{Q}$ ratio}

\begin{tabular}{llr} 
Name of Company & Country & Q ratio \\
\hline H \& M Hennes \& Mauritz AB & Sweden & 6.465 \\
\hline Tractor Supply Company & U.S. & 5.176 \\
\hline BiM Birleşik Mağazalar A.Ş. & Turkey & 4.759 \\
\hline Next plc & U.K. & 4.679 \\
\hline Inditex, S.A. & Spain & 4.570 \\
\hline Dollar Tree, Inc. & U.S. & 4.514 \\
\hline Nike, Inc. & U.S. & 4.353 \\
\hline The TJX Companies, Inc. & U.S. & 4.338 \\
\hline Ross Stores, Inc. & U.S. & 4.331 \\
\hline Fast Retailing Co., Ltd. & Japan & 3.951 \\
\hline
\end{tabular}




\begin{tabular}{|c|c|}
\hline \multicolumn{2}{|l|}{$Q$ ratio by country } \\
\hline Canada & 0.520 \\
\hline China & 0.252 \\
\hline France & 0.502 \\
\hline Germany & 0.282 \\
\hline Hong Kong & 0.838 \\
\hline Japan & 0.427 \\
\hline Mexico & 1.023 \\
\hline Russia & 1.086 \\
\hline South Africa & 0.568 \\
\hline South Korea & 0.295 \\
\hline Sweden & 3.473 \\
\hline UK & 0.634 \\
\hline US & 1.819 \\
\hline \multicolumn{2}{|l|}{ Q ratio by region } \\
\hline Africa/Middle East & 1.315 \\
\hline Asia Pacific & 0.469 \\
\hline Asia Pacfic less Japan & 0.510 \\
\hline Europe & 0.847 \\
\hline Latin America & 0.837 \\
\hline North America & 1.760 \\
\hline \multicolumn{2}{|l|}{ Q ratio by retail segment } \\
\hline Apparel/Footwear specialty & 1.727 \\
\hline Convenience & 0.533 \\
\hline Department stores & 0.599 \\
\hline Discount department stores & 0.601 \\
\hline Discount stores & 0.997 \\
\hline Drug stores & 1.250 \\
\hline Electronics & 2.392 \\
\hline Electronics less Apple & 0.512 \\
\hline Home improvement & 1.696 \\
\hline Hypermarkets & 0.657 \\
\hline Other specialty & 1.212 \\
\hline Supermarkets & 0.804 \\
\hline \multicolumn{2}{|l|}{ Q ratio by product sector } \\
\hline Apparel and accessories & 1.238 \\
\hline Diversified & 0.468 \\
\hline FMCG & 0.817 \\
\hline Hardlines/leisure & 2.085 \\
\hline Composite $\mathrm{Q}$ for all retailers & 1.130 \\
\hline Average $\mathrm{Q}$ for all retailers & 1.410 \\
\hline
\end{tabular}




\section{Study methodology and data sources}

Companies were included in the Top 250 Global Powers of Retailing based on their non-auto retail revenue for fiscal year 2013 (encompasses fiscal years ended through June 2014). To be included on the list, a company does not have to derive the majority of its revenue from retailing so long as its retailing activity is large enough to qualify. Private equity and other investment firms are not considered as retail entities in this report - only the individual operating companies.

A number of sources were consulted to develop the Top 250 list. The principal data sources for financial and other company information were annual reports, SEC filings, and information found in company press releases and fact sheets or on company websites. If company-issued information was not available, other public-domain sources were used, including trade journal estimates, industry analyst reports, and various business information databases.

Much of the data for non-U.S. retailers came from Planet Retail, a leading provider of global intelligence, analysis, news, and data covering more than 9,000 retail operations across 211 markets around the world. Planet Retail has offices in London, Frankfurt, New York, Tokyo, Hong Kong, and Qingdao, China. For more information please visit www. planetretail.net.

Group Revenue reflects the consolidated net revenue of a retailer's parent company, whether or not that company itself is primarily a retailer. Similarly, the income/loss and total assets figures also reflect the consolidated results of the parent organization. If a privately held company reports gross turnover only, this figure is reported as Group Revenue and footnoted as "g." Revenue figures do not include operations in which a company has only a minority interest.

The Retail Revenue figures in this report reflect only the retail portion of the company's consolidated net revenue. As a result, they may reflect adjustments to reported revenue figures to exclude non-retail operations. Retail Revenue includes foodservice sales if foodservice is sold as one of the merchandise offerings inside the retail store or if restaurants are located within the company's stores, but excludes separate foodservice/restaurant operations where it is possible to break them out. Retail Revenue also includes sales of services related to the company's retail activities, such as alterations, repair, maintenance, installation, etc.; fuel sales; and membership fees. However, retailers that derive the majority of their retail revenue from the sale of motor fuel are considered to be primarily gas stations and are excluded from Top 250 consideration.

Revenue figures do not include the retail banner sales of franchised, licensed, or independent cooperative member stores; they do include royalties and franchising or licensing fees. Group Revenue includes wholesale sales to such networked operations - both member stores and other supplied stores.
Retail Revenue includes wholesale sales to affiliated/member stores but excludes traditional wholesale or other business-to-business revenue (except where such revenue is derived from retail stores), where it is possible to break them out.

For e-commerce companies, retail revenue includes only direct B2C sales where the company is the seller of record. It excludes the sales of third-party sellers as well as third-party seller fees and commissions.

In order to provide a common base from which to rank companies by their Retail Revenue results, fiscal year 2013 revenues for nonU.S. companies were converted to U.S. dollars. Exchange rates, therefore, have an impact on the results. OANDA.com is the source for the exchange rates. The average daily exchange rate corresponding to each company's fiscal year was used to convert that company's results to U.S. dollars. Individual companies' 2013 year-over-year growth rate and 2008-2013 compound annual growth rate (CAGR) for Retail Revenue, however, were calculated in each company's local currency.

\section{Group financial results}

This report uses sales-weighted composites rather than simple arithmetic averages as the primary measure for understanding group financial results. Therefore, results of larger companies contribute more to the composite than do results of smaller companies. Because the data has been converted to U.S. dollars for ranking purposes and to facilitate comparison among groups, composite growth rates also have been adjusted to correct for currency movement. While these composite results generally behave in a similar fashion to arithmetic averages, they provide better representative values for benchmarking purposes.

Group financial results are based only on companies with data. Not all data elements were available for all companies. Top 250 companies that did not derive the majority of their revenue from retail operations were excluded from the calculation of group profitability ratios as their consolidated profits mostly reflect nonretail activities.

It should also be noted that the financial information used for each company in a given year is accurate as of the date the financial report was originally issued. Although a company may have restated prior-year results to reflect a change in its operations or as a result of an accounting change, such restatements are not reflected in this data.

This study is not an accounting report. It is intended to provide an accurate reflection of market dynamics and their impact on the structure of the retailing industry over a period of time. As a result of these factors, growth rates for individual companies may not correspond to other published results. 


\section{Contacts}

Retail contacts for Deloitte Touche Tohmatsu Limited (DTTL) and its member firms

Global
Deloitte Global Leader, Consumer
Business
Antoine de Riedmatten
aderiedmatten@deloitte.fr
Deloitte Global Sector Leader,
Retail
Vicky Eng
veng@deloitte.com
Chief Global Economist
Ira Kalish
ikalish@deloitte.com
Consumer Business Marketing
Kathryn Cordes
kcordes@deloitte.com

North America

Canada

Ryan Brain

rbrain@deloitte.ca

United States

Alison Paul

Deloitte Consulting LLP

alpaul@deloitte.com
Europe, Middle East and Africa (EMEA)

Belgium

Olivier de Groote

oldegroote@deloitte.com

Central Europe

Aaron Martin

aamartin@deloittece.com

Czech Republic

Martin Tesař

mtesar@deloittece.com

Denmark

Jesper Povlsen

jepovlsen@deloitte.dk

East Africa

John Kiarie

jkiarie@deloitte.co.ke

Finland

Jussi Sairanen

jussi.sairanen@deloitte.fi

France

Stephane Rimbeuf

srimbeuf@deloitte.fr

Germany

Karsten Hollasch

khollasch@deloitte.de

Greece

Dimitris Koutsopoulos

dkoutsopoulos@deloitte.gr

Ireland

Kevin Sheehan

kesheehan@deloitte.ie

Israel

Israel Nakel

inakel@deloitte.co.il

Italy

Dario Righetti

drighetti@deloitte.it

Middle East

Herve Ballantyne

hballantyne@deloitte.com

Netherlands

Erik Nanninga

enanninga@deloitte.nl

Norway

Jonathan Farnell

jfarnell@deloitte.no
Poland

Dariusz Kraszewski

dkraszewski@deloittece.com

Portugal

Luís Belo

Ibelo@deloitte.pt

Russia/CIS

Vladimir Biryukov

vbiryukov@deloitte.ru

South Africa

Andre Dennis

adennis@deloitte.co.za

Spain

Fernando Pasamon

fpasamon@deloitte.es

Sweden

Joakim Torbjorn

jtorbjorn@deloitte.se

Switzerland

Howard da Silva

hdasilva@deloitte.ch

Turkey

Ozgur Yalta

oyalta@deloitte.com

United Kingdom

Ian Geddes

igeddes@deloitte.co.uk

West Africa

Alain Penanguer

apenanguer@deloitte.fr

Latin America

Latin America Consumer Business

Leader

Reynaldo Saad

rsaad@deloitte.com

Argentina/LATCO

Daniel Varde

dvarde@deloitte.com

Brazil

Reynaldo Saad

rsaad@deloitte.com

Chile

Cristian Alvarez

cralvarez@deloitte.com

Colombia

Juan Sanchez

jsancheznino@deloitte.com
Mexico

Luis Castañeda

castaneda@deloittemx.com

Omar Camacho

ocamacho@deloittemx.com

Peru

Francisco Revelo

frevelo@deloitte.com

Venezuela

Ignacio Rodriguez

igrodriguez@deloitte.com

Asia Pacific

Asia Pacific Consumer Business

Leader

Haruhiko Yahagi

hyahagi@tohmatsu.co.jp

Australia

David White

davidwhite@deloitte.com.au

China

David Lung

dalung@deloitte.com.cn

Indonesia

Jose Sabater

josabater@deloitte.com

Japan

Haruhiko Yahagi

hyahagi@tohmatsu.co.jp

Korea

Jae Hoon Lee

jaehoolee@deloitte.com

Malaysia

Jeffrey Soo

jefsoo@deloitte.com

Philippines

Bonifacio Lumacang

blumacang@deloitte.com

SEA \& Singapore

Eugene Ho

eugeneho@deloitte.com

Taiwan

Jason Ke

jasonke@deloitte.com.tw

Thailand

Manoon Manusook

mmanusook@deloitte.com

Vietnam

Nguyen Vu Duc

nguyenvu@deloitte.co 
Notes 

Deloitte refers to one or more of Deloitte Touche Tohmatsu Limited, a UK private company limited by guarantee, and its network of member firms, each of which is a legally separate and independent entity. Please see www.deloitte.com/about for a detailed description of the legal structure of Deloitte Touche Tohmatsu Limited and its member firms.

Deloitte provides audit, tax, consulting, and financial advisory services to public and private clients spanning multiple industries. With a globally connected network of member firms in more than 150 countries, Deloitte brings world-class capabilities and high quality service to clients, delivering the insights they need to address their most complex business challenges. Deloitte has in the region of 200,000 professionals, all committed to becoming the standard of excellence.

This publication is for internal distribution and use only among personnel of Deloitte Touche Tohmatsu Limited, its member firms, and its and their affiliates.

None of Deloitte Touche Tohmatsu Limited, Deloitte Global Services Limited, Deloitte Global Services Holdings Limited, the Deloitte Touche Tohmatsu Verein, any of their member firms, or any of the foregoing's affiliates shall be responsible for any loss whatsoever sustained by any person who relies on this publication.

(c) 2015. For more information, contact Deloitte Touche Tohmatsu Limited.

Designed and produced by The Creative Studio at Deloitte, London. 40350A 